WSRC-RP-93-1545

Analysis of Volatile Organic Compounds (VOCs) in A/M Area Crouch Branch (Cretaceous) Aquifer Characterization Samples: 1993 (U)

December 6, 1993

Westinghouse Savannah River Company

Savannah River Technology Center

Environmental Sciences Section

Aiken SC 29808

Prepared for the U. S. Department of Energy under Contract No. DE-AC09-89SR18035 


\section{DISCLAMMER}

This report was prepared by Westinghouse Savannah River Company (WSRC) for the United States Department of Energy under Contract No. DE-AC09-89SR 18035 and is an account of work performed under that contract. Neither the United States Department of Energy, nor WSRC, nor any of their employees makes any warranty, expressed or implied, or assumes any legal liability or responsibility for the accuracy, completeness, or usefulness, of any information, apparatus, or product or process disclosed herein or represents that its use will not infringe privately owned rights. Reference herein to any specific commercial product, process, or service by trademark, name, manufacturer or otherwise does not necessarily constitute or imply endorsement, recommendation, or favoring of same by WSRC or by the United States Government or any agency thereof. The views and opinions of the authors expressed herein do not necessarily state or reflect those of the United States Government or any agency thereof. 
Analysis of Volatile Organic Compounds (VOCs) in A/M Area Croc.ch Branch (Cretaceous) Aquifer Characterization Samples: 1993 (U)

December 6, 1993

B. B. Looney J. S. Haselow

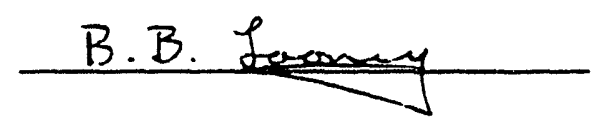
M. A. Keenan

R. Van Pelt

C. A. Eddy-Dilek

J. Rossabi

J. L. Simmons

Westinghouse Savannah River Company

Savannah River Technology Center

Environmental Sciences Section

Aiken SC 29808

Approved by:

DBran fare

D. B. Moore-Shedrow, Section Manager Environmental Sciences Section

Prepared for the U. S. Department of Energy under Contract No. DE-AC09-89SR18035 
Analysis of Volatiıe Organic Compounds (VOCs) in A/M Area Crouch Branch (Cretaceous) Aquifer Characterization Samples: 1993

\section{Summary}

Bulk samples ( 3 to $5 \mathrm{~cm}^{3}$ ) were collected during the A/M Area Crouch Branch (Cretaceous) Aquifer Characterization (Phase I) Program. The samples were analyzed for chlorinated VOCs by the Savannah River Technology Center (SRTC) and MicroSeeps Ltd. All samples were sealed in the field immediately upon retrieval of the core and subsampling. A total of 113 samples locations were selected for analysis. The Environmental Sciences Section (ESS) of SRTC analyzed all locations in duplicate (226 samples). MicroSeeps Ltd was selected as the quality assurance (QA) check laboratory. MicroSeeps Ltd analyzed 40 locations with 4 duplicates (44 samples). The samples were collected from seven boreholes in $A / M$ Area in the interval from 200 feet deep to the total depth of the boring ( 360 feet deep nominal); samples were collected every 10 feet within this interval. The sampling zone corresponds approximately to the Crouch Branch Aquifer in A/M Area. The overall A/M Area Crouch Branch Aquifer characterization objectives, a brief description of $\mathrm{A} / \mathrm{M}$ Area geology and hydrology, and the sample locations, field notes, driller lithologic logs, and required procedural documentation are presented in WSRC (1993).

The primary analytes were trichloroethylene (TCE) and tetrachloroethylene (PCE). The sample data are summarized in Tables 1 and 2. All of the VOC concentrations were relatively low (less than approximately $0.1 \mathrm{ug} / \mathrm{g}$ ) in this study. Approximately $70 \%$ of the samples were below detection $(<0.001 \mathrm{ug} / \mathrm{g})$ for both compounds. The maximum TCE concentration was $0.094 \mathrm{ug} / \mathrm{g}$ and the maximum PCE concentration was $0.030 \mathrm{ug} / \mathrm{g}$. No detectable solvents were measured in MBCSB-1, MBCSB-6, and MBCSB-7. TCE was detected in each sample collected from MBCSB-3. Note that this boring is located near monitoring well cluster MSB-47, a location previously identified as a principal entry point of VOCs into the groundwater system. The TCE values in MBCSB-3 ranged from $0.003 \mathrm{ug} / \mathrm{g}$ to $0.051 \mathrm{ug} / \mathrm{g}$. No PCE was detected in this boring. The rest of the borings (MBCSB-2, MBCSB-4, and MBCSB-5) showed a similar distribution of detected VOCs. These cores showed detectable VOCs near 220 feet deep and in the lower portion of the boring (e.g., 320 to 360 feet deep). Based on examination of the field geologic descriptions, these depths represent water bearing zones between or just above fine grained sediments (aquitards). The expected range of water concentrations associated with each depth discrete sample analyzed during the study is tabulated based on previus comparisons in $A / M$ Area. These findings and additional observations are discussec' below.

The quality assurance checks of the data suggest that the results are of high quality. There was a high degree of concordance between the intralaboratory duplicate samples. Additionally, the sample locations analyzed by both SRTC and MicroSeeps Ltd using different analytical methods yielded similar concentrations. SRTC analyzed the samples using a static headspace method developed to support SRS groundwater VOC remediation activities and documented in the EPA report on innovative VOC analysis methods (Looney et al., 1993). MicroSeeps Ltd utilized the 
new (currently draft) EPA Method 5035 based on purge and trap of the suspended sediment sample using a special vial and laboratory apparatus (EPA, draft). The comparison data suggest that the heating of the sample vials in the SRTC sample set slightly improved the consistency and recovery of VOCs from the samples. Heating of the vials to encourage recovery of the VOCs into the purge gas is currently being examined by MicroSeeps to improve the performance of the new EPA method.

\section{Methods}

Depth Discrete bulk sediment samples were collected for chlorinated VOC analysis from the A/M Area Crouch Branch (Cretaceous) Aquifer Characterization. Samples were collected at ten foot intervals between 200 feet deep and the total depth of the boring ( 360 feet deep nominal) using a $94 \mathrm{~mm}$ double tube wireline core. The cores were extruded into polyvinyl chloride troughs for immediate VOC sampling, followed by measuring, lithologic description, and archiving (WSRC, 1993). The driller and geological oversight informed the sampler of the drilling progress so that each target core could be sampled promptly. Bentonite based drilling fluids were utilized during the mud rotary drilling. Following extrusion of the samplers collected 3 to $5 \mathrm{~cm}^{3}$ of the saturated bulk sediment using a $12 \mathrm{~mm}$ diameter sample tube (modified plastic syringe). As discussed below, all samples were sealed in the field immediately upon retrieval of the core and subsampling. The sampling syringes were decontaminated between samples by brushing and rinsing with isopropanol followed by deionized water.

The samples collected $f-r$ this study were analyzed using two different methods to assist in assuring the quality of the data. All samples were analyzed by SRTC using a static headspace method. This method, originally developed to support VOC remediation technology demonstrations in A/M Area (Looney, 1993). A portion of the samples (approximately 20\%) were analyzed by MicroSeeps Ltd as a quality check using the new EPA Method 5035 (EPA, draft). Each of these methods is summarized below. A full description of the SRTC method is provided in Appendix C and a copy of the draft EPA Method 5035 is provided in Appendix D.

\section{Headspace Method (SRTC Analysis)}

The depth discrete bulk sediment sample was extruded into a $22.5 \mathrm{~mL}$ borosilicate vial. Using a pipet, $5 \mathrm{~mL}$ of deionized water was added as a suspending solution and the vial was sealed by crimping an aluminum cap around a teflon lined butyl rubber septum. The sample was labeled and placed in a chilled ice chest for later analysis. Prior to field sampling, we determined the average weight of a sealed headspace vial containing $5 \mathrm{~mL}$ of suspending solution. Upon receipt of the sample vials from the field, the capped vials containing sediment samples were weighed. The amount of sediment in each vial was determined by subtracting the average weight from the sample weight. Each vial was then analyzed using a Hewlett Packard (HP) 5890 gas chromatograph (GC) equipped with an electron capture detector, a HP 19395 headspace sampler, and a $60 \mathrm{~m}$ widebore capillary column coated with a nonpolar silicone phase. The flow and temperature conditions recommended by the manufacturers were used. The instrument was 
calibrated using vials containing water of known concentrations. The conditions in the vials (headspace volume, suspension volume, etc.) were standardized to maintain the proportionality between the sediment concentration and the headspace concentration. For example, the heated (70 degrees Celsius) bath in the headspace bath maximizes the transfer of VOC into the headspace for analysis.

Standards were run corresponding to each of the seven cores collected in this study. All of the standards data, the equation for the relevant portion of the standard curve, and graphs of the data for TCE and PCE are provided in Appendix D. The concentration in each sample was estimated using the response factor from the appropriate portion of the calibration curve. All values are reported in units of micrograms of VOC per gram of bulk sediment (ug/g). The method is described further in Looney et al. (1993) -- Appendix C.

\section{Modified Purge and Trap Analysis (MicroSeeps Ltd Analysis)}

MicroSeeps Ltd recently implemented the new EPA Method 5035 (draft) in their laboratory and is providing this service to SRS under task order agreement. Samples were collected as described above and placed in specially designed vials. The vials allow sealing in the field and estimation of sample weight in the same fashion as the headspace method. The special sample container provides access from both ends and is designed to be mounted into a recently developed purge and trap GC interface (DynaSoils). At the present time, the capability to reproducibly heat the vials prior to analysis is not implemented in the method. The samples were analyzed using a HP GC equipped with both electron capture and flame ionization detectors. The concentration in each sample was determined using the response factor from the appropriate portion of the calibration curve. All values were originally reported in units of nanograms of VOC per gram of bulk sediment (ng/g). The summary table (Table 1) presents all data in units of ug/g for consistency. The method is described further in EPA (draft) -- Appendix D.

\section{Results}

The data for each sample location (core-depth) are summarized in Tables 1 and 2 for both laboratories. The full SRTC Dataset showing all duplicate results and supporting raw data are provided in Appendix A. The full MicroSeeps dataset is provided in Appendix B.

The primary analytes were trichloroethylene (TCE) and tetrachloroethylene (PCE). MicroSeeps Ltd analyzed for the following additional VOCs -- vinyl chloride, methylene chloride, trans 1,2 dichloroethylene, chloroform, 1,1,1 trichloroethane, and carbon tetrachloride. The detection limits for both laboratories are listed in Table 2. Those compounds marked with an asterisk were not detected in the samples analyzed.

The sample data are summarized in Tables 1 and 2. All of the VOC concentrations were relatively low (less than approximately $0.1 \mathrm{ug} / \mathrm{g}$ ) in this study. Approximately $70 \%$ of the samples were below detection $(<0.001 \mathrm{ug} / \mathrm{g})$ for both primary compounds. The maximum TCE 
concentration was $0.094 \mathrm{ug} / \mathrm{g}$ and the maximum PCE concentration was $0.030 \mathrm{ug} / \mathrm{g}$. No detectable solvents were measured in MBCSB-1, MBCSB-6, and MBCSB-7. TCE was detected in each sample collected from MBCSB-3. Note that this boring is located near monitoring well cluster MSB-47, a location previously identified as a principal entry point of VOCs into the groundwater system. The TCE values in MBCSB-3 ranged from $0.003 \mathrm{ug} / \mathrm{g}$ to $0.051 \mathrm{ug} / \mathrm{g}$. No PCE was detected in this boring. The rest of the borings (MBCSB-2, MBCSB-4, and MBCSB-5) showed a similar distribution of detected VOCs. These cores showed detectable VOCs near 220 feet deep and in the lower portion of the boring (e.g., 320 to 360 feet deep). Based on examination of the field geologic descriptions, these depths represent water bearing zones between or just above fine grained sediments (aquitards). The concentrations in cores MBCSB-2, MBCSB-4, and MBCSB-5 ranged from $<0.001 \mathrm{ug} / \mathrm{g}$ to $0.094 \mathrm{ug} / \mathrm{g}$. Previous studies in A/M Area at SRS indicated that the depth discrete bulk sediment concentrations are related to the groundwater concentrations at the sample depth (Eddy et al., 1991). Typically, the groundwater concentration (in ug/L) is 2000 to 4000 times the bulk sediment concentration (in $\mathrm{ug} / \mathrm{g}$ ). Using these factors, the approximate range of expected groundwater concentration at each depth is calculated in Table 3.

The various quality assurance checks of the data suggest that the results are of high quality. There was a high degree of concordance between the intralaboratory duplicate samples. To assess the concordance of the duplicates, we calculated the normalized spread of each pair (S) in percent where:

$$
\begin{aligned}
& S=100 \times[(\text { maximum value }- \text { average value }) /(\text { average value })] \\
& =100 \times[(\text { average value }- \text { minimum value }) /(\text { average value })]
\end{aligned}
$$

Figure 1 shows the percent spread as a function of average concentration for all 113 samples analyzed by SRTC. The average spread was $13.5 \%$ for TCE and $8 \%$ for PCE. The typical minimum and maximum values were typically within the average $\mathrm{S} \%$ of the mean values.

The sample locations analyzed by both SRTC and MicroSeeps Ltd using different analytical methods yielded similar concentrations. The range of values for the interlaboratory comparison data sets were:

SRTC: TCE: $<0.001$ to $0.094 \mathrm{ug} / \mathrm{g}$
PCE: $<0.001$ to $0.030 \mathrm{ug} / \mathrm{g}$
MicroSeeps Ltd

TCE: $<0.001$ to $0.078 \mathrm{ug} / \mathrm{g}$

PCE: $<0.001$ to $0.015 \mathrm{ug} / \mathrm{g}$

Figure 2 is a graph of the results from the two laboratories. There was an exact concordance between the laboratories in identifying the highest VOC concentrations and a good concordance in identifying samples below detection. SRTC analyzed the samples using a static headspace method developed to support SRS groundwater VOC remediation activities and documented in the EPA report on innovative VOC analysis methods (Looney, 1993). MicroSeeps Ltd utilized the new (currently draft) EPA Method 5035 based on purge and trap of the suspended sediment 
sample using a special vial and laboratory apparatus (EPA, draft). The comparison data suggest that the heating of the sample vials in the SRTC sample set slightly improved the consistency and recovery of VOCs from the samples. Note for example the slightly lower PCE values in the MicroSeeps Ltd data. Also, the samples with the largest discrepancy, MBCSB-4 near 240 feet deep were clayey in nature. Both of these observations are consistent with the expected behavior of the methods based on the impact of heating. Heating of the vials to encourage recovery of the VOCs into the purge gas is currently being examined by MicroSeeps to improve the performance of the new EPA method.

Six of the samples analyzed by MicroSeeps Ltd contained traces $(0.001$ to $0.004 \mathrm{ug} / \mathrm{g})$ of chloroform. This has been observed in past drilling studies at SRS in which depth discrete VOC samples were collected. Two causes have been identified for this observation. First, several natural organics coelute with chloroform increasing the likelihood of a false positive (note that the field notes indicate an trace OVA reading associated with this core suggesting the possibility of the presence of natural organic compounds). Second, chloroform is an indication (tracer) of minor infiltration of drilling fluids. The potable water used to mix the drilling mud, typical of most drinking water supplies, is treated using standard chlorination methods that result in generation of low concentrations of trihalomethanes (e.g., chloroform) during treatment. Two of the MicroSeeps Ltd analyses from MBCSB-1 (310' and 350') contain trace levels of TCE, PCE, and 1,1,1 trichloroethane. The system blank for this core contains these same constituents in the same ratio. Thus, these two sample analyses should be viewed as suspect and are likely the result of a trace sample blank contamination associated with the initiation of the new EPA Method 5035. The MicroSeeps Ltd samples appear to be of extremely high quality, and the new method appears promising and viable for sample analysis of SRS sediments.

\section{References}

Eddy, C. A., B. B. Looney, J. M. Dougherty, T. C. Hazen, and D. S. Kaback, 1991. Characterization of the Geology, Geochemistry, Hydrology and Microbiology of the In-Situ Air Stripping Demonstration Site at SRS. WSRC-RD-91-21, Westinghouse Savannah River Company, Savannah River Site, Aiken SC 29808.

EPA (Environmental Protection Agency), draft. Method 5035: Modified Purge-and-Trap and Extraction for Volatile Organics in Soil and Waste Samples. EPA Office of Solid and Hazardous Waste, Washington DC.

Looney, B. B., C. A. Eddy, and W. R. Sims, 1993. Evaluation of Headspace Method for Volatile Constituents in Soils and Sediments. in Measuring and Interpreting VOCs in Soils: State of the Art and Research Needs, Us Environmental Protection Agency, Environmental Monitoring Systems Laboratory, Las Vegas NV 89193.

WSRC (Westinghouse Savannah River Company), 1993. A/M Area Crouch Branch (Cretaceous) Aquifer Characterization - Phase I: Soil Coring Report. WSRC-RP-93-1241, Westinghouse Savannah River Company, Savannah River Site, Aiken SC 29808. 
Table 1. Summary of A/M Area Crouch Branch (Cretaceous) Aquifer Characterization VOC Data

\begin{tabular}{|c|c|c|c|c|c|c|c|}
\hline & & \multicolumn{2}{|c|}{ SRTC DATA } & \multicolumn{3}{|c|}{ MicroSeeps Data } & \\
\hline Core & $\begin{array}{l}\text { Depth } \\
\text { feet }\end{array}$ & $\begin{array}{c}\text { TCE } \\
\text { avg. conc. } \\
u g / g \\
\end{array}$ & $\begin{array}{c}\text { PCE } \\
\text { avg. conc. } \\
\text { ug/g } \\
\end{array}$ & $\begin{array}{l}\text { TCE } \\
\text { ug/g } \\
\end{array}$ & $\begin{array}{l}\mathrm{PCE} \\
\mathrm{ug} / \mathrm{g} \\
\end{array}$ & $\begin{array}{c}\text { chloroform } \\
4 \mathrm{~g} / \mathrm{g} \\
\end{array}$ & $\begin{array}{c}1,1,1 \\
\text { trichloroethane } \\
\mathrm{ug} / \mathrm{g} \\
\end{array}$ \\
\hline MBCSB-1 & $200^{\prime}$ & $<0.001$ & $<0.001$ & & & & \\
\hline MBCSB-1 & $210^{\prime}$ & $<0.001$ & $<0.001$ & & & & \\
\hline MBCSB-1 & $220^{\prime}$ & $<0.001$ & $<0.001$ & & & & \\
\hline MBCSB-1 & $230^{\circ}$ & $<0.001$ & $<0.001$ & & & & \\
\hline MBCSB-1 & $240^{\prime}$ & $<0.001$ & $<0.001$ & & & & \\
\hline MBCSB-1 & $250^{\prime}$ & $<0.001$ & $<0.001$ & & & & \\
\hline MBCSB-1 & $260^{\prime}$ & $<0.001$ & $<0.001$ & & & & \\
\hline MBCSB-1 & $270^{\prime}$ & $<0.001$ & $<0.001$ & & & & \\
\hline MBCSB-1 & $280^{\prime}$ & $<0.001$ & $<0.001$ & & & & \\
\hline MBCSB-1 & $290^{\prime}$ & $<0.001$ & $<0.001$ & & & & \\
\hline MBCSB-1 & $300^{\prime}$ & $<0.001$ & $<0.001$ & & & & \\
\hline MBCSB-1 & $310^{\circ}$ & $<0.001$ & $<0.001$ & 0.008 & 0.002 & $<0.001$ & 0.025 \\
\hline MBCSB-1 & $320^{\prime}$ & $<0.001$ & $<0.001$ & $<0.001$ & $<0.001$ & $<0.001$ & $<0.001$ \\
\hline MBCSB-1 & $330^{\prime}$ & $<0.001$ & $<0.001$ & $<0.001$ & $<0.001$ & $<0.001$ & $<0.001$ \\
\hline MBCSB-1 & $340^{\prime}$ & $<0.001$ & $<0.001$ & $<0.001$ & $<0.001$ & $<0.001$ & $<0.001$ \\
\hline MBCSB-1 & $350^{\prime}$ & $<0.001$ & $<0.001$ & 0.005 & 0.001 & $<0.001$ & 0.014 \\
\hline MBCSB-1 & $360^{\prime}$ & $<0.001$ & $<0.001$ & $<0.001$ & $<0.001$ & $<0.001$ & $<0.001$ \\
\hline replicate & $360^{\prime}$ & $<0.001$ & $<0.001$ & $<0.001$ & $<0.001$ & $<0.001$ & $<0.001$ \\
\hline MBCSB-2 & $200^{\prime}$ & 0.001 & $<0.001$ & $<0.001$ & $<0.001$ & $<0.001$ & $<0.001$ \\
\hline replicate & $200^{\prime}$ & 0.001 & $<0.001$ & $<0.001$ & $<0.001$ & $<0.001$ & $<0.001$ \\
\hline MBCSB-2 & $210^{\prime}$ & 0.003 & $<0.001$ & $<0.001$ & $<0.001$ & $<0.001$ & $<0.001$ \\
\hline MBCSB-2 & $220^{\prime}$ & $<0.001$ & $<0.001$ & $<0.001$ & $<0.001$ & $<0.001$ & $<0.001$ \\
\hline MBCSB-2 & $230^{\prime}$ & $<0.001$ & $<0.001$ & $<0.001$ & $<0.001$ & $<0.001$ & $<0.001$ \\
\hline MBCSB-2 & $240^{\prime}$ & $<0.001$ & $<0.001$ & $<0.001$ & $<0.001$ & $<0.001$ & $<0.001$ \\
\hline MBCSB-2 & $250^{\prime}$ & $<0.001$ & $<0.001$ & $<0.001$ & $<0.001$ & $<0.001$ & $<0.001$ \\
\hline MBCSB-2 & $260^{\prime}$ & $<0.001$ & $<0.001$ & $<0.001$ & $<0.001$ & $<0.001$ & $<0.001$ \\
\hline MBCSB-2 & $270^{\prime}$ & $<0.001$ & $<0.001$ & $<0.001$ & $<0.001$ & $<0.001$ & $<0.001$ \\
\hline MBCSB-2 & $280^{\prime}$ & $<0.001$ & $<0.001$ & $<0.001$ & $<0.001$ & $<0.001$ & $<0.001$ \\
\hline MBCSB-2 & $290^{\circ}$ & $<0.001$ & $<0.001$ & $<0.001$ & $<0.001$ & $<0.001$ & $<0.001$ \\
\hline MBCSB-2 & $300^{\prime}$ & $<0.001$ & $<0.001$ & $<0.001$ & $<0.001$ & $<0.001$ & $<0.001$ \\
\hline replicate & $300^{\prime}$ & $<0.001$ & $<0.001$ & $<0.001$ & $<0.001$ & $<0.001$ & $<0.001$ \\
\hline MBCSB-2 & $310^{\prime}$ & $<0.001$ & $<0.001$ & $<0.001$ & $<0.001$ & $<0.001$ & $<0.001$ \\
\hline MBCSB-2 & $320^{\prime}$ & 0.002 & $<0.001$ & $<0.001$ & $<0.001$ & $<0.001$ & $<0.001$ \\
\hline MBCSB-2 & $330^{\prime}$ & $<0.001$ & $<0.001$ & $<0.001$ & $<0.001$ & $<0.001$ & $<0.001$ \\
\hline MBCSB-2 & $340^{\prime}$ & 0.002 & $<0.001$ & $<0.001$ & $<0.001$ & $<0.001$ & $<0.001$ \\
\hline MBCSB-2 & $350^{\prime}$ & $<0.001$ & $<0.001$ & $<0.001$ & $<0.001$ & $<0.001$ & $<0.001$ \\
\hline MBCSB-2 & $360^{\prime}$ & $<0.001$ & $<0.001$ & $<0.001$ & $<0.001$ & $<0.001$ & $<0.001$ \\
\hline
\end{tabular}


Table 1. Summary of $\mathbf{A} / \mathbf{M}$ Area Crouch Branch (Cretaceous) Aquifer Characterization VOC Data (continued)

SRTC DATA

\begin{tabular}{|c|c|c|c|c|c|c|c|}
\hline & \multicolumn{3}{|c|}{ SRTC DATA } & \multicolumn{3}{|c|}{ MicroSeeps Data } & \\
\hline Core & $\begin{array}{l}\text { Depth } \\
\text { feet }\end{array}$ & $\begin{array}{c}\text { TCE } \\
\text { avg. conc. } \\
\text { ug/g } \\
\end{array}$ & $\begin{array}{c}\text { PCE } \\
\text { avg. conc. } \\
\text { ug/g } \\
\end{array}$ & $\begin{array}{l}\text { TCE } \\
\text { ue/g } \\
\end{array}$ & $\begin{array}{l}\text { PCE } \\
\text { ug/g } \\
\end{array}$ & $\begin{array}{c}\text { chloroform } \\
\mathrm{ug} / \mathrm{g} \\
\end{array}$ & $\begin{array}{c}1,1,1 \\
\begin{array}{c}\text { trichloroethane } \\
\text { ug/g }\end{array} \\
\end{array}$ \\
\hline MBCSB-3 & $200^{\prime}$ & 0.015 & $<0.001$ & & & & \\
\hline MBCSB-3 & $210^{\prime}$ & 0.007 & $<0.001$ & & & & \\
\hline MBCSB-3 & $220^{\prime}$ & 0.006 & $<0.001$ & . & & & \\
\hline MBCSB-3 & $230^{\prime}$ & 0.003 & $<0.001$ & & & & \\
\hline MBCSB-3 & $240^{\prime}$ & 0.003 & $<0.001$ & & & & \\
\hline MBCSB-3 & $250^{\prime}$ & 0.051 & $<0.001$ & & & & \\
\hline MBCSB-3 & $260^{\prime}$ & 0.011 & $<0.001$ & & & & \\
\hline MBCSB-3 & $270^{\prime}$ & 0.009 & $<0.001$ & & & & \\
\hline MBCSB-4 & $200^{\prime}$ & $<0.001$ & $<0.001$ & $<0.001$ & $<0.001$ & 0.004 & $<0.001$ \\
\hline replicate & $200^{\prime}$ & $<0.001$ & $<0.001$ & $<0.001$ & $<0.001$ & 0.003 & $<0.001$ \\
\hline MBCSB-4 & $210^{\prime}$ & $<0.001$ & $<0.001$ & $<0.001$ & $<0.001$ & 0.002 & $<0.001$ \\
\hline MBCSB-4 & $220^{\prime}$ & 0.001 & $<0.001$ & $<0.001$ & $<0.001$ & 0.002 & $<0.001$ \\
\hline MBCSB-4 & $230^{\prime}$ & 0.024 & $<0.001$ & $<0.001$ & $<0.001$ & 0.002 & $<0.001$ \\
\hline MBCSB-4 & $240^{\prime}$ & 0.026 & 0.003 & $<0.001$ & $<0.001$ & $<0.001$ & $<0.001$ \\
\hline MBCSB-4 & $250^{\prime}$ & 0.001 & $<0.001$ & $<0.001$ & $<0.001$ & $<0.001$ & $<0.001$ \\
\hline MBCSB-4 & $260^{\circ}$ & $<0.001$ & $<0.001$ & $<0.001$ & $<0.001$ & $<0.001$ & $<0.001$ \\
\hline MBCSB-4 & $270^{\prime}$ & $<0.001$ & $<0.001$ & $<0.001$ & $<0.001$ & $<0.001$ & $<0.001$ \\
\hline MBCSB-4 & $280^{\prime}$ & $<0.001$ & $<0.001$ & $<0.001$ & $<0.001$ & $<0.001$ & $<0.001$ \\
\hline MBCSB-4 & $290^{\prime}$ & $<0.001$ & $<0.001$ & $<0.001$ & $<0.001$ & $<0.001$ & $<0.001$ \\
\hline MBCSB-4 & $300^{\prime}$ & $<0.001$ & $<0.001$ & $<0.001$ & $<0.001$ & $<0.001$ & $<0.001$ \\
\hline replicate & $300^{\prime}$ & $<0.001$ & $<0.001$ & $<0.001$ & $<0.001$ & $<0.001$ & $<0.001$ \\
\hline MBCSB-4 & $310^{\prime}$ & 0.002 & $<0.001$ & 0.001 & $<0.001$ & $<0.001$ & 0.001 \\
\hline $\mathrm{MBCSB}-4$ & $320^{\prime}$ & 0.003 & 0.013 & 0.001 & 0.006 & $<0.001$ & 0.001 \\
\hline MBCSB-4 & $330^{\prime}$ & 0.003 & 0.016 & & & & \\
\hline MBCSB-4 & $340^{\prime}$ & 0.040 & 0.030 & 0.042 & 0.015 & 0.001 & $<0.001$ \\
\hline MBCSB-4 & $350^{\prime}$ & 0.054 & 0.030 & & & & \\
\hline $\mathrm{MBCSB}-4$ & $360^{\circ}$ & 0.094 & 0.011 & 0.078 & 0.004 & $<0.001$ & $<0.001$ \\
\hline
\end{tabular}


Table 1. Summary of A/M Area Crouch Branch (Cretaceous) Aquifer Characterization VOC Data (continued)

SRTC DATA

\begin{tabular}{|c|c|c|c|c|c|c|c|}
\hline & & SKIC DA IA & & & roseef & & \\
\hline Core & $\begin{array}{l}\text { Depth } \\
\text { feet }\end{array}$ & $\begin{array}{c}\text { TCE } \\
\text { avg. conc. } \\
\text { ug/g } \\
\end{array}$ & $\begin{array}{c}\text { PCE } \\
\text { avg. conc. } \\
\text { ug/g } \\
\end{array}$ & $\begin{array}{l}\text { TCE } \\
\mathrm{ug} / \mathrm{g}\end{array}$ & $\begin{array}{l}\text { PCE } \\
\mathrm{ug} / \mathrm{g}\end{array}$ & $\begin{array}{c}\text { chloroform } \\
u g / g \\
\end{array}$ & $\begin{array}{c}1,1,1 \\
\text { trichloroethane } \\
u g / g\end{array}$ \\
\hline MBCSB-5 & $200^{\prime}$ & 0.003 & $<0.001$ & & & & \\
\hline MBCSB-5 & $210^{\prime}$ & $<0.001$ & $<0.001$ & & & & \\
\hline MBCऽB-5 & $220^{\prime}$ & $<0.001$ & $<0.001$ & & & & \\
\hline MBCSB-5 & $230^{\prime}$ & $<0.001$ & $<0.001$ & & & & \\
\hline MBCSB-5 & $240^{\prime}$ & $<0.001$ & $<0.001$ & & & & \\
\hline MBCSB-5 & $250^{\prime}$ & 0.013 & $<0.001$ & & & & \\
\hline MBCSB-5 & $260^{\prime}$ & 0.017 & $<0.001$ & & & & \\
\hline MBCSB-5 & $270^{\prime}$ & $<0.001$ & $<0.001$ & & & & \\
\hline MBCSB-5 & $280^{\prime}$ & $<0.001$ & $<0.001$ & & & & \\
\hline MBCSB-5 & $290^{\prime}$ & $<0.001$ & $<0.001$ & & & & \\
\hline MBCSB-5 & $300^{\prime}$ & $<0.001$ & $<0.001$ & & & & \\
\hline MBCSB-5 & $310^{\circ}$ & $<0.001$ & $<0.001$ & & & & \\
\hline MBCSB-5 & $320^{\prime}$ & $<0.001$ & $<0.001$ & & & & \\
\hline MBCSB.5 & $330^{\prime}$ & $<0.001$ & $<0.001$ & & & & \\
\hline MBCSB-5 & $340^{\prime}$ & $<0.001$ & $<0.001$ & & & & \\
\hline MBCSB-5 & $350^{\prime}$ & 0.020 & 0.004 & & & & \\
\hline MBCSB-5 & $360^{\prime}$ & 0.046 & 0.013 & & & & \\
\hline MBCSB-5 & $365^{\prime}$ & 0.015 & 0.007 & & & & \\
\hline MBCSB-6 & $200^{\prime}$ & $<0.001$ & $<0.001$ & & & & \\
\hline MBCSB-6 & $210^{\prime}$ & $<0.001$ & $<0.001$ & & & & \\
\hline MBCSB-6 & $220^{\prime}$ & $<0.001$ & $<0.001$ & & & & \\
\hline MBCSB-6 & $230^{\prime}$ & $<0.001$ & $<0.001$ & & & & \\
\hline MBCSB-6 & $240^{\prime}$ & $<0.001$ & $<0.001$ & & & & \\
\hline MBCSB-6 & $250^{\prime}$ & $<0.001$ & $<0.001$ & & & & \\
\hline MBCSB-6 & $260^{\prime}$ & $<0.001$ & $<0.001$ & & & & \\
\hline MBCSB-6 & $270^{\prime}$ & $<0.001$ & $<0.001$ & & & & \\
\hline MBCSB-6 & $280^{\prime}$ & $<0.001$ & $<0.001$ & & & & \\
\hline MBCSB-6 & $290^{\prime}$ & $<0.001$ & $<0.001$ & & & & \\
\hline MBCSB-6 & $300^{\prime}$ & $<0.001$ & $<0.001$ & & & & \\
\hline MBCSB-6 & $310^{\prime}$ & $<0.001$ & $<0.001$ & & & & \\
\hline MBCSB-6 & $320^{\prime}$ & $<0.001$ & $<0.001$ & & & & \\
\hline MBCSB-6 & $330^{\prime}$ & $<0.001$ & $<0.001$ & & & & \\
\hline MBCSB-6 & $340^{\prime}$ & $<0.001$ & $<0.001$ & & & & \\
\hline MBCSB-6 & $350^{\prime}$ & $<0.001$ & $<0.001$ & & & & \\
\hline MBCSB-6 & $360^{\prime}$ & $<0.001$ & $<0.001$ & & & & \\
\hline
\end{tabular}


Table 1. Summary of AMM Area Crouch Branch (Cretaceous) Aquifer Characterization VOC Data (continued)

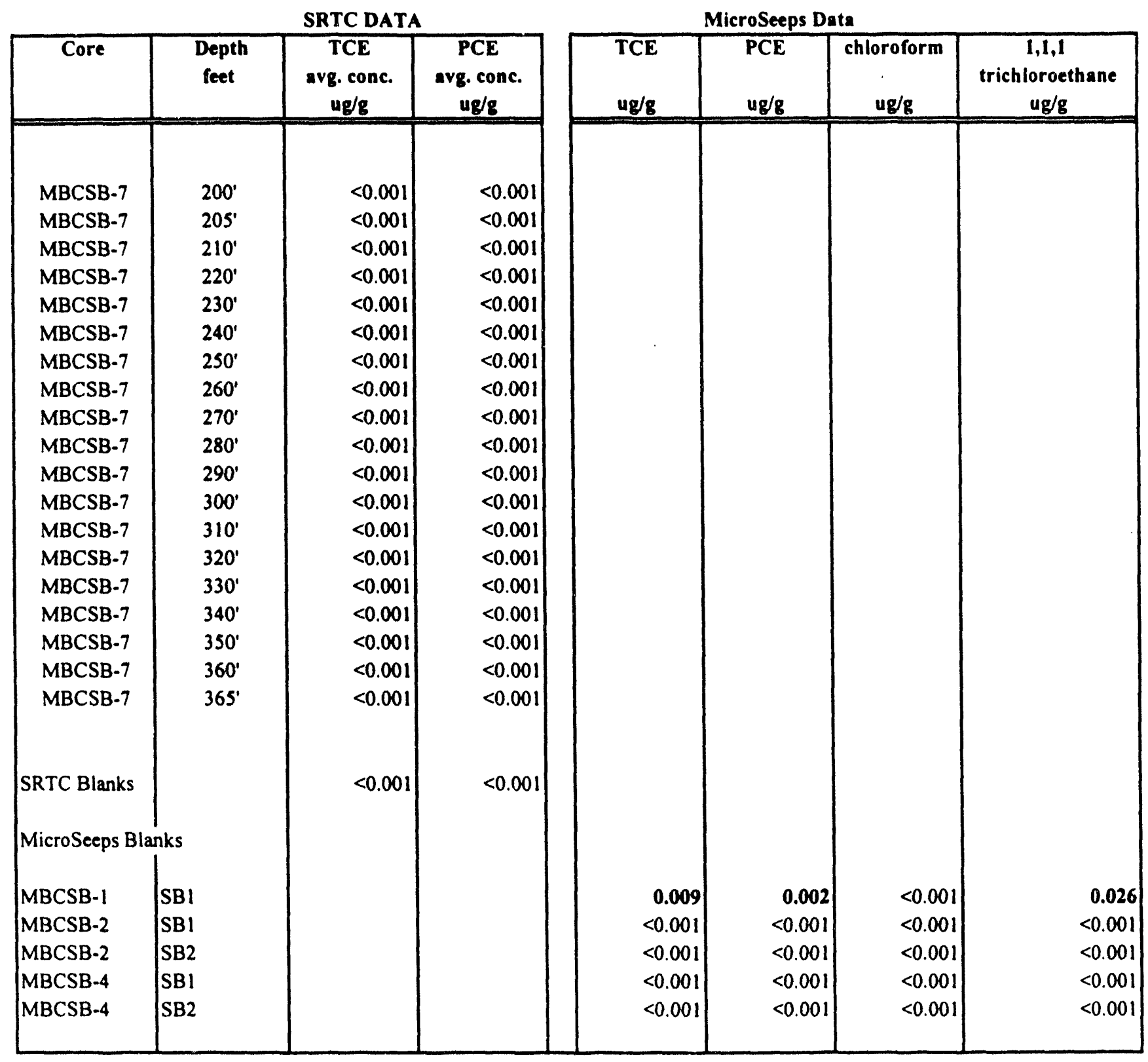


Table 1. Summary of A/M Area Crouch Branch (Cretaceous) Aquifer Characierization VOC Data (continued)

Corehole Details (WSRC, 1993)

\begin{tabular}{|ccccc|}
\hline Core & $\begin{array}{c}\text { SRS Northing } \\
\text { feet }\end{array}$ & $\begin{array}{c}\text { SRS Easting } \\
\text { feet }\end{array}$ & $\begin{array}{c}\text { Surface } \\
\text { Elevation } \\
\text { feet above MSL }\end{array}$ & $\begin{array}{c}\text { Total Depth } \\
\text { feet }\end{array}$ \\
\hline MBCSB-1 & 108450 & 52780 & 372.0 & 365 \\
MBCSB-2 & 107750 & 52975 & 380.2 & 365 \\
MBCSB-3 & 107178 & 52035 & 367.2 & 270 \\
MBCSB-4 & 105008 & 51445 & 381.2 & 365 \\
MBCSB-5 & 103983 & 51641 & 370.5 & 365 \\
MBCSB-6 & 104750 & 50600 & 328.6 & 365 \\
MBCSB-7 & 100850 & 48750 & 365 & \\
na = not available & & & & \\
\end{tabular}


Table 2. Detection Limits and Identification of VOCs not Detected in Study

Headspace Method (SRTC Analysis)

trichloroethylene $\quad<0.001 \mathrm{ug} / \mathrm{g}$

tetrachloroethylene $\quad<0.001 \mathrm{ug} / \mathrm{g}$

Purge and Trap Method (MicroSeeps Ltd Analysis)

trichloroethylene $\quad<0.001 \mathrm{ug} / \mathrm{g}$

tetrachloroethylene $\quad<0.001 \mathrm{ug} / \mathrm{g}$

vinyl chloride $\quad<0.001 \mathrm{ug} / \mathrm{g}$

methylene chloride $\quad<0.020 \mathrm{ug} / \mathrm{g}$ *

trans 1,2 dichloroethylene $<0.020 \mathrm{ug} / \mathrm{g}$ *

chloroform $<0.001 \mathrm{ug} / \mathrm{g}$

$1,1,1$ trichloroethane $\quad<0.001 \mathrm{ug} / \mathrm{g}^{* *}$

carbon tetrachloride $\quad<0.001 \mathrm{ug} / \mathrm{g}$ *

* = not detected in any sample

** = detected in only 2 samples and the associated system blank 
Table 3. Water Concentrations Estimated from Depth Discrete Bulk Sediment Results

\begin{tabular}{|c|c|c|c|c|c|c|c|c|c|}
\hline \multicolumn{2}{|r|}{$\cdot$} & \multicolumn{2}{|c|}{ SEDIMENT CONCENTRATION } & \multicolumn{6}{|c|}{ ESTIMATED WATER CONCENTRATIONS } \\
\hline Well ID & $\begin{array}{l}\text { Depth } \\
\text { feet }\end{array}$ & $\begin{array}{c}\text { TCE } \\
\text { ave. conc. } \\
\text { ug/s }\end{array}$ & $\begin{array}{c}\text { PCE } \\
\text { avg. conc. } \\
\text { ug/g }\end{array}$ & estims & $\begin{array}{l}\text { TCE } \\
d \cos \\
d / 2\end{array}$ & range & estims & $\begin{array}{l}\mathrm{PCE} \\
\mathrm{col} \\
\mathrm{de} / \mathrm{L}\end{array}$ & range \\
\hline MBCSB-1 & $200^{\prime}-360^{\prime}$ & $<0.001$ & $<0.001$ & $<2$ & to & $<5$ & $<2$ & to & $<5$ \\
\hline MBCSB-2 & $200^{\circ}$ & 0.001 & $<0.001$ & 2 & to & 6 & $<2$ & to & $<5$ \\
\hline MBCSB-2 & $210^{\circ}$ & 0.003 & $<0.001$ & $\mathbf{s}$ & to & 13 & $<2$ & to & $<5$ \\
\hline MBCSB-2 & $2: 010310^{\prime}$ & $<0.001$ & $<0.001$ & $<2$ & to & $<5$ & $<2$ & to & $<5$ \\
\hline MBCSB-2 & $320^{\prime}$ & 0.002 & $<0.001$ & 3 & to & 8 & $<2$ & to & $<5$ \\
\hline MBCSB-2 & $330^{\prime}$ & $<0.001$ & $<0.001$ & $<2$ & to & $<5$ & $<2$ & 10 & $<5$ \\
\hline MBCSB-2 & $340^{\circ}$ & 0.002 & $<0.001$ & 4 & 10 & 10 & $<2$ & io & $<5$ \\
\hline MBCSB-2 & $350^{\circ}-360^{\circ}$ & $<0.001$ & $<0.001$ & $<2$ & to & $<9$ & $<2$ & 10 & $<5$ \\
\hline MBCSB.3 & $200^{\prime}$ & 0.015 & $<0.001$ & 30 & 10 & 75 & $<2$ & to & $<5$ \\
\hline MBCSB-3 & $210^{\prime}$ & 0.007 & $<0.001$ & 14 & to & 34 & $<2$ & to & $<5$ \\
\hline MBCSB-3 & $220^{\prime}$ & 0.006 & $<0.001$ & 12 & to & 31 & $<2$ & 10 & $<5$ \\
\hline MBCSB-3 & $230^{\prime}$ & 0.003 & $<0.001$ & 5 & to & 13 & $<2$ & 10 & $<5$ \\
\hline MBCSB-3 & $240^{\prime}$ & 0.003 & $<0.001$ & 7 & to & 17 & $<2$ & 10 & $<5$ \\
\hline MBCSB-3 & $250^{\circ}$ & 0.051 & $<0.001$ & 101 & to & 253 & $<2$ & 10 & $<5$ \\
\hline MBCSB-3 & $260^{\prime}$ & 0.011 & $<0.001$ & 22 & to & 54 & $<2$ & 10 & $<5$ \\
\hline $\mathrm{MBCSB}-3$ & $270^{\circ}$ & 0.009 & $<0.001$ & 18 & to & 45 & $<2$ & to & $<5$ \\
\hline $\mathrm{MBCSB}-4$ & $200^{\prime}-210^{\prime}$ & $<0.001$ & $<0.001$ & $<2$ & to & $<5$ & $<2$ & to & $<5$ \\
\hline MBCSB-4 & $220^{\prime}$ & 0.001 & $<0.001$ & 2 & to & 6 & $<2$ & to & $<5$ \\
\hline MBCSB-A & $230^{\circ}$ & 0.024 & $<0.001$ & 49 & to & 122 & $<2$ & to & $<s$ \\
\hline MBCSB-4 & $240^{\circ}$ & 0.026 & 0.003 & 53 & to & 132 & 6 & to & 14 \\
\hline MBCSB-A & $250^{\prime}$ & 0.001 & $<0.001$ & 2 & to & 5 & $<2$ & to & $<5$ \\
\hline $\mathrm{MBCSB}-4$ & $260^{\prime}-300^{\circ}$ & $<0.001$ & $<0.001$ & $<2$ & to & $<5$ & $<2$ & to & $<5$ \\
\hline $\mathrm{MBCSB}-4$ & $310^{\circ}$ & 0.002 & $<0.001$ & 4 & to & 10 & $<2$ & to & $<s$ \\
\hline MBCSB-4 & $320^{\prime}$ & 0.003 & 0.013 & 6 & to & 14 & 26 & to & 64 \\
\hline $\mathrm{MBCSB}-4$ & $330^{\circ}$ & 0.003 & 0.016 & $\mathbf{s}$ & 10 & 13 & 31 & to & 79 \\
\hline $\mathrm{MBCSB}-4$ & $340^{\circ}$ & 0.040 & 0.030 & 80 & to & 199 & 61 & to & 152 \\
\hline MBCSB-4 & $350^{\circ}$ & 0.054 & 0.030 & 109 & to & 272 & 60 & to & 151 \\
\hline $\mathrm{MBCSB}-4$ & $360^{\circ}$ & 0.094 & 0.011 & 188 & to & $\$ 70$ & 21 & 10 & 53 \\
\hline MBCSB-5 & $200^{\prime}$ & 0.003 & $<0.001$ & 6 & 10 & 15 & $<2$ & to & $<5$ \\
\hline MBCSB-S & $210^{\prime}-240^{\prime}$ & $<0.001$ & $<0.001$ & $<2$ & to & $<5$ & $<2$ & to & $<5$ \\
\hline MBCSB-5 & $250^{\prime}$ & 0.013 & $<0.001$ & 25 & to & 63 & $<2$ & to & $<5$ \\
\hline MBCSB-S & $260^{\prime}$ & 0.017 & $<0.001$ & 33 & to & 83 & $<2$ & to & $<5$ \\
\hline MBCSB-S & $270^{\circ}-340^{\circ}$ & $<0.001$ & $<0.001$ & $<2$ & 10 & $<5$ & $<2$ & to & $<5$ \\
\hline MBCSB-5 & $350^{\prime}$ & 0.020 & 0.004 & 41 & 10 & 102 & 8 & 10 & 19 \\
\hline MBCSB-5 & $360^{\prime}$ & 0.046 & 0.013 & 92 & to & 230 & 26 & 10 & 65 \\
\hline MBCSB-5 & $365^{\prime}$ & 0.015 & 0.007 & 29 & to & 73 & 14 & to & 35 \\
\hline MBCSB-6 & $200^{\prime} \cdot 360^{\circ}$ & $<0.001$ & $<0.001$ & $<2$ & 10 & $<5$ & $<2$ & to & $<5$ \\
\hline MBCSB-7 & $200^{\circ}-360^{\prime}$ & $<0.001$ & $<0.001$ & $<2$ & 10 & $<5$ & $<2$ & to & $<s$ \\
\hline MBCSB-7 & $365^{\prime}$ & $<0.001$ & $<0.001$ & $<2$ & 10 & $<5$ & $<2$ & to & $<5$ \\
\hline
\end{tabular}

Where a depth range is indicated, discrete samples were collected every 10 feet. 


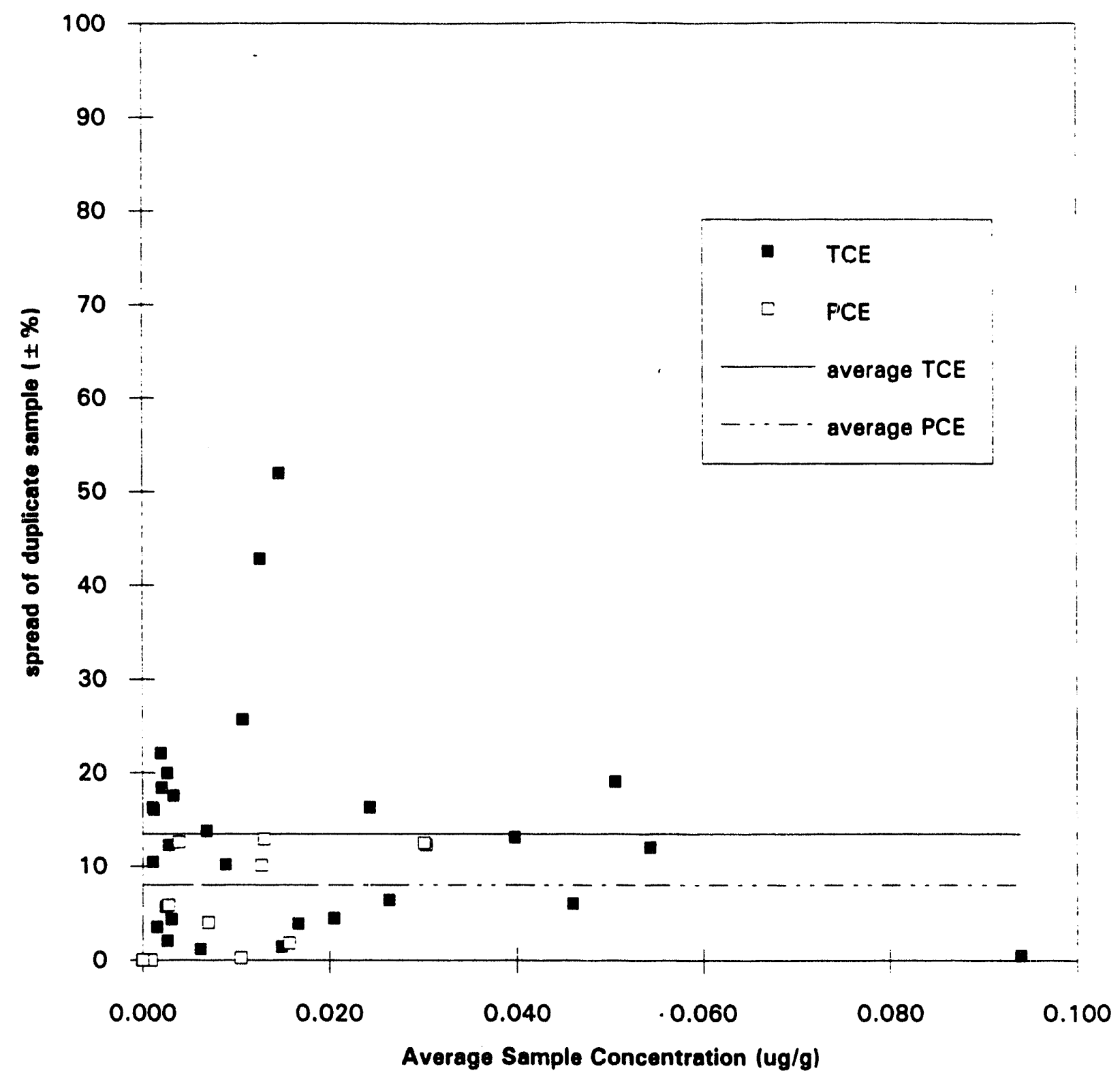

Figure 1. Evaluation of the concordance of the $\mathbf{1 1 3}$ duplicate samples analyzed by the headspace method (SRTC) 
Interlaboratory Comparison

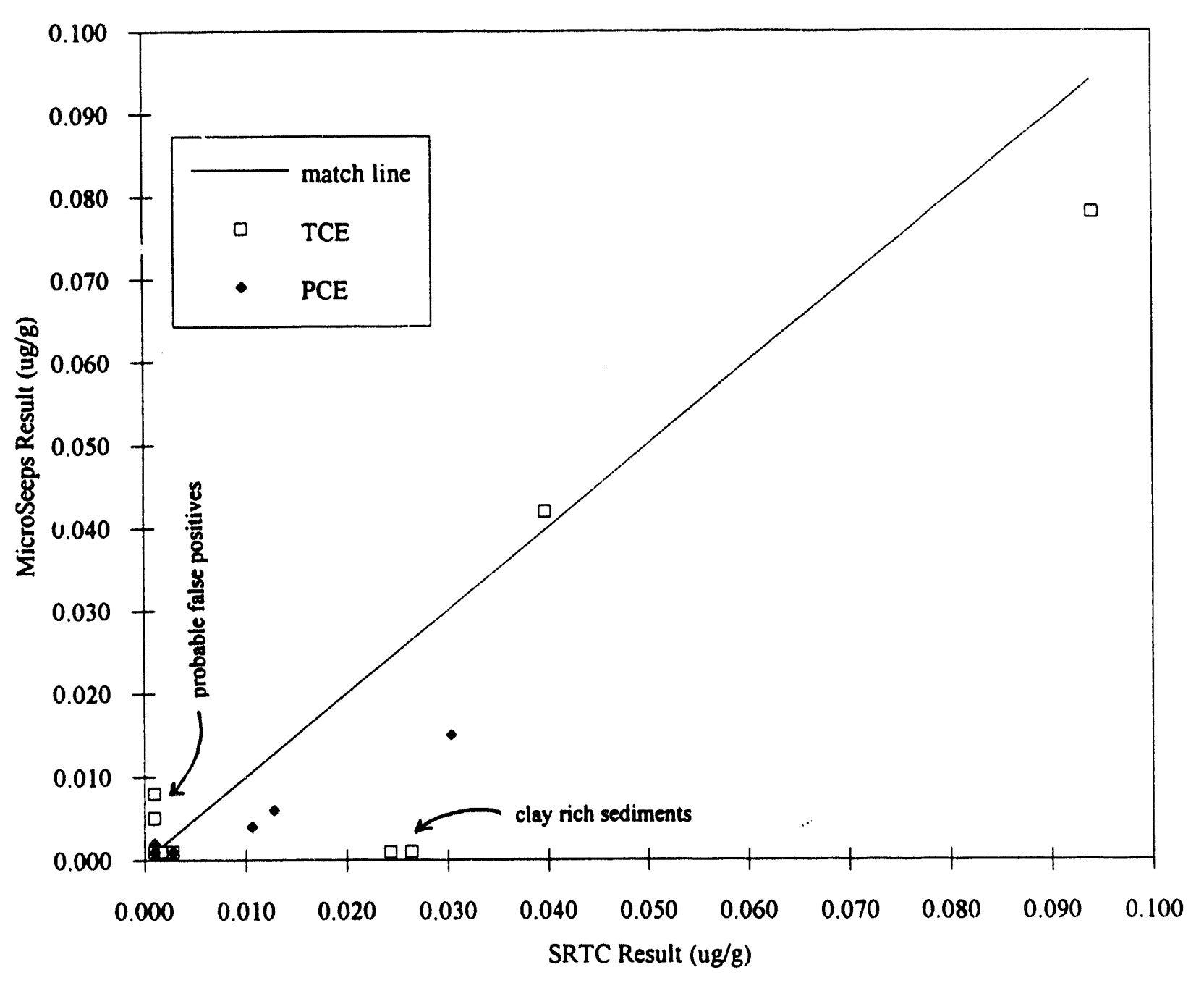

Figure 2. Evaluation of the concordance of the interlaboratory comparison samples. 


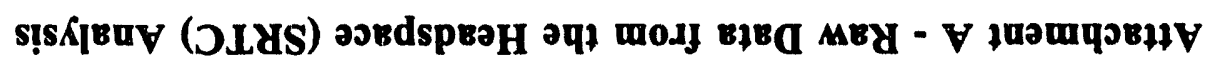




\begin{tabular}{|c|c|c|c|c|c|c|c|c|c|}
\hline Well ID & $\begin{array}{l}\text { Depth } \\
\text { feet }\end{array}$ & $\begin{array}{c}\text { Vial } \\
\text { Mass } \\
8 \\
\end{array}$ & $\begin{array}{c}\text { TCE } \\
\text { counts }\end{array}$ & $\begin{array}{c}\text { PCE } \\
\text { counts }\end{array}$ & $\begin{array}{c}\text { Sediment } \\
\text { Mass } \\
8 \\
\end{array}$ & $\begin{array}{r}\mathrm{TCE} \\
\text { conc. } \\
\mathrm{ug} / \mathrm{g} \\
\end{array}$ & $\begin{array}{l}\text { PCE } \\
\text { conc. } \\
\text { ug/g } \\
\end{array}$ & $\begin{array}{c}\text { TCE } \\
\text { avg. conc. } \\
\text { ug/g } \\
\end{array}$ & $\begin{array}{c}\text { PCE } \\
\text { avg. conc. } \\
\mathrm{ug} / \mathrm{g} \\
\end{array}$ \\
\hline MBCSB-1 & $200^{\prime}$ & 25,59 & ND & $1.571 E+03$ & 2.99 & $<0.001$ & $<0.001$ & $<0.001$ & $<0.001$ \\
\hline MBCSB-1 & $200^{\circ}$ & 25.94 & ND & ND & 3.34 & $<0.001$ & $<0.001$ & & \\
\hline MBCSB-1 & $210^{\prime}$ & 24.71 & ND & ND & 2.11 & $<0.001$ & $<0.001$ & $<0.001$ & $<0.001$ \\
\hline MBCSB-1 & $210^{\circ}$ & 25.89 & ND & ND & 3.29 & $<0.001$ & $<0.001$ & & \\
\hline MBCSB-1 & $220^{\prime}$ & 24.48 & ND & ND & 1.88 & $<0.001$ & $<0.001$ & $<0.001$ & $<0.001$ \\
\hline MBCSB-1 & $220^{\prime}$ & 24.53 & ND & ND & 1.93 & $<0.001$ & $<0.001$ & & \\
\hline MBCSB-1 & $230^{\prime}$ & 26.13 & ND & ND & 3.53 & $<0.001$ & $<0.001$ & $<0.001$ & $<0.001$ \\
\hline MBCSB-1 & $230^{\prime}$ & 26.52 & ND & ND & 3.92 & $<0.001$ & $<0.001$ & & \\
\hline MBCSB-1 & $240^{\circ}$ & 26.32 & ND & ND & 3.72 & $<0.001$ & $<0.001$ & $<0.001$ & $<0.001$ \\
\hline MBCSB-1 & $240^{\circ}$ & 25.82 & ND & ND & 3.22 & $<0.001$ & $<0.001$ & & \\
\hline MBCSB-1 & $250^{\prime}$ & 25.96 & ND & ND & 3.36 & $<0.001$ & $<0.001$ & $<0.001$ & $<0.001$ \\
\hline MBCSB-1 & $250^{\prime}$ & 25.88 & ND & ND & 3.28 & $<0.001$ & $<0.001$ & & \\
\hline MBCSB-1 & $260^{\circ}$ & 25.79 & ND & ND & 3.19 & $<0.001$ & $<0.001$ & $<0.001$ & $<0.001$ \\
\hline MBCSB-1 & $260^{\prime}$ & 25.99 & ND & ND & 3.39 & $<0.001$ & $<0.001$ & & \\
\hline MBCSB-1 & $270^{\prime}$ & 25.83 & ND & ND & 3.23 & $<0.001$ & $<0.001$ & $<0.001$ & $<0.001$ \\
\hline MBCSB-1 & $270^{\prime}$ & 26.41 & ND & ND & 3.81 & $<0.001$ & $<0.001$ & & \\
\hline MBCSB-I & $280^{\prime}$ & 26.05 & ND & ND & 3.45 & $<0.001$ & $<0.001$ & $<0.001$ & $<0.001$ \\
\hline MBCSB-1 & $280^{\prime}$ & 25.46 & ND & ND & 2.86 & $<0.001$ & $<0.001$ & & \\
\hline MBCSB-1 & $290^{\prime}$ & 26.48 & ND & ND & 3.88 & $<0.001$ & $<0.001$ & $<0.001$ & $<0.001$ \\
\hline MBCSB-1 & $290^{\prime}$ & 25.96 & ND & ND & 3.36 & $<0.001$ & $<0.001$ & & \\
\hline MBCSB-1 & $300^{\prime}$ & 26.53 & ND & ND & 3.93 & $<0.001$ & $<0.001$ & $<0.001$ & $<0.001$ \\
\hline MBCSB-1 & $300^{\prime}$ & 26.31 & ND & ND & 3.71 & $<0.001$ & $<0.001$ & & \\
\hline MBCSB-1 & $310^{\prime}$ & 25.39 & ND & ND & 2.79 & $<0.001$ & $<0.001$ & $<0.001$ & $<0.001$ \\
\hline MBCSB-1 & $310^{\prime}$ & 25.64 & ND & ND & 3.04 & $<0.001$ & $<0.001$ & & \\
\hline MBCSB-1 & $320^{\prime}$ & 26.88 & ND & $3.125 E+03$ & 4.28 & $<0.001$ & $<0.001$ & $<0.001$ & $<0.001$ \\
\hline MBCSB-I & $320^{\prime}$ & 26.35 & ND & ND & 3.75 & $<0.001$ & $<0.001$ & & \\
\hline MBCSB-1 & $330^{\prime}$ & 27.62 & ND & ND & 5.02 & $<0.001$ & $<0.001$ & $<0.001$ & $<0.001$ \\
\hline MBCSB-1 & $330^{\circ}$ & 26.85 & ND & ND & 4.25 & $<0.001$ & $<0.001$ & & \\
\hline MBCSB-1 & $340^{\prime}$ & 26.93 & ND & ND & 4.33 & $<0.001$ & $<0.001$ & $<0.001$ & $<0.001$ \\
\hline MBCSB-1 & $340^{\prime}$ & 26.08 & ND & ND & 3.48 & $<0.001$ & $<0.001$ & & \\
\hline MBCSB-1 & $350^{\prime}$ & 27.39 & ND & ND & 4.79 & $<0.001$ & $<0.001$ & $<0.001$ & $<0.001$ \\
\hline MBCSB-1 & $350^{\prime}$ & 27.61 & ND & ND & 5.01 & $<0.001$ & $<0.001$ & & \\
\hline MBCSB-1 & $360^{\prime}$ & 27.45 & ND & $4.798 E+03$ & 4.85 & $<0.001$ & $<0.001$ & $<0.001$ & $<0.001$ \\
\hline MBCSB-1 & $360^{\prime}$ & 27.77 & ND & $6.803 E+04$ & 5.17 & $<0.001$ & $<0.001$ & & \\
\hline
\end{tabular}




\begin{tabular}{|c|c|c|c|c|c|c|c|c|c|}
\hline Well ID & $\begin{array}{l}\text { Depth } \\
\text { feet }\end{array}$ & $\begin{array}{c}\text { Vial } \\
\text { Mass } \\
8 \\
\end{array}$ & $\begin{array}{c}\text { TCE } \\
\text { counts }\end{array}$ & $\begin{array}{c}\text { PCE } \\
\text { counts }\end{array}$ & $\begin{array}{c}\text { Sediment } \\
\text { Mass } \\
g\end{array}$ & $\begin{array}{l}\text { TCE } \\
\text { conc. } \\
\text { ug/g } \\
\end{array}$ & $\begin{array}{l}\text { PCE } \\
\text { conc. } \\
\text { ug/g } \\
\end{array}$ & $\begin{array}{c}\text { TCE } \\
\text { avg. conc. } \\
\text { ug/g } \\
\end{array}$ & $\begin{array}{c}\text { PCE } \\
\text { avg. conc. } \\
\text { ug/g }\end{array}$ \\
\hline MBCSB-2 & $200^{\prime}$ & 25.35 & $4.511 E+04$ & $3.495 \mathrm{E}+03$ & 2.75 & 0.001 & $<0.001$ & 0.001 & $<0.001$ \\
\hline MBCSB-2 & $200^{\prime}$ & 25.22 & $3.109 E+04$ & $4.057 E+03$ & 2.62 & 0.001 & $<0.001$ & & \\
\hline MBCSB-2 & $210^{\prime}$ & 26.08 & $9.485 E+04$ & $4.848 E+03$ & 3.48 & 0.002 & $<0.001$ & 0.003 & $<0.001$ \\
\hline MBCSB-2 & $210^{\prime}$ & 26.34 & $1.143 E+05$ & $4.069 E+03$ & 3.74 & 0.003 & $<0.001$ & & \\
\hline MBCSB-2 & $220^{\prime}$ & 25.61 & $1.099 E+04$ & $3.466 E+03$ & 3.01 & $<0.001$ & $<0.001$ & $<0.001$ & $<0.001$ \\
\hline MBCSB-2 & $220^{\prime}$ & 25.87 & $1.359 \mathrm{E}+04$ & $2.348 E+03$ & 3.27 & $<0.001$ & $<0.001$ & & \\
\hline MBCSB-2 & $230^{\prime}$ & 26.24 & $2.096 \mathrm{E}+03$ & $3.018 E+03$ & 3.64 & $<0.001$ & $<0.001$ & $<0.001$ & $<0.001$ \\
\hline MBCSB-2 & $230^{\prime}$ & 25.80 & $1.738 E+03$ & $2.510 \mathrm{E}+03$ & 3.20 & $<0.001$ & $<0.001$ & & \\
\hline MBCSB-2 & $240^{\prime}$ & 26.02 & $3.505 E+03$ & $1.645 E+03$ & 3.42 & $<0.001$ & $<0.001$ & $<0.001$ & $<0.001$ \\
\hline MBCSB-2 & $240^{\circ}$ & 26.52 & $2.622 \mathrm{E}+03$ & $2.344 E+03$ & 3.92 & $<0.001$ & $<0.001$ & & \\
\hline MBCSB-2 & $250^{\circ}$ & 26.08 & $3.614 E+03$ & $2.037 \mathrm{E}+03$ & 3.48 & $<0.001$ & $<0.001$ & $<0.001$ & $<0.001$ \\
\hline MBCSB-2 & $250^{\prime}$ & 25.98 & $3.879 E+03$ & $1.995 E+03$ & 3.38 & $<0.001$ & $<0.001$ & & \\
\hline MBCSB-2 & $260^{\prime}$ & 26.43 & $1.683 E+03$ & $1.916 \mathrm{E}+03$ & 3.83 & $<0.001$ & $<0.001$ & $<0.001$ & $<0.001$ \\
\hline $\mathrm{MBCSB}-2$ & $260^{\circ}$ & 25.98 & $1.810 \mathrm{E}+03$ & N.D. & 3.38 & $<0.001$ & $<0.001$ & & \\
\hline MBCSB-2 & $270^{\circ}$ & 25.63 & N.D. & $1.556 \mathrm{E}+03$ & 3.03 & $<0.001$ & $<0.001$ & $<0.001$ & $<0.001$ \\
\hline MBCSB-2 & $270^{\prime}$ & 26.31 & N.D. & N.D. & 3.71 & $<0.001$ & $<0.001$ & & \\
\hline MBCSB-2 & $280^{\prime}$ & 27.01 & N.D. & N.D. & 4.41 & $<0.001$ & $<0.001$ & $<0.001$ & $<0.001$ \\
\hline MBCSB-2 & $280^{\prime}$ & 26.86 & N.D. & $1.583 E+03$ & 4.26 & $<0.001$ & $<0.001$ & & \\
\hline MBCSB-2 & $290^{\prime}$ & 25.84 & N.D. & $1.513 \mathrm{E}+03$ & 3.24 & $<0.001$ & $<0.001$ & $<0.001$ & $<0.001$ \\
\hline MBCSB-2 & $290^{\prime}$ & 25.60 & N.D. & $1.598 \mathrm{E}+03$ & 3.00 & $<0.001$ & $<0.001$ & & \\
\hline MBCSB-2 & $300^{\prime}$ & 25.33 & N.D. & N.D. & 2.73 & $<0.001$ & $<0.001$ & $<0.001$ & $<0.001$ \\
\hline MBCSB-2 & $300^{\prime}$ & 25.47 & N.D. & N.D. & 2.87 & $<0.001$ & $<0.001$ & & \\
\hline MBCSB-2 & $310^{\prime}$ & 25.76 & $1.063 E+04$ & N.D. & 3.16 & $<0.001$ & $<0.001$ & $<0.001$ & $<0.001$ \\
\hline MBCSB-2 & $310^{\prime}$ & 26.27 & $9.116 \mathrm{E}+03$ & N.D. & 3.67 & $<0.001$ & $<0.001$ & & \\
\hline MBCSB-2 & $320^{\prime}$ & 25.41 & $5.273 E+04$ & N.D. & 2.81 & 0.002 & $<0.001$ & 0.002 & $<0.001$ \\
\hline MBCSB-2 & $320^{\prime}$ & 25.44 & $4.966 E+04$ & N.D. & 2.84 & 0.002 & $<0.001$ & & \\
\hline MBCSB-2 & $330^{\prime}$ & 26.55 & N.D. & N.D. & 3.95 & $<0.001$ & $<0.001$ & $<0.001$ & $<0.001$ \\
\hline MBCSB-2 & $330^{\prime}$ & 27.12 & N.D. & $1.394 E+03$ & 4.52 & $<0.001$ & $<0.001$ & & \\
\hline MBCSB-2 & $340^{\prime}$ & 25.94 & $6.574 E+04$ & N.D. & 3.34 & 0.002 & $<0.001$ & 0.002 & $<0.001$ \\
\hline MBCSB-2 & $340^{\prime}$ & 26.18 & $1.022 E+05$ & N.D. & 3.58 & 0.002 & $<0.001$ & & \\
\hline MBCSB-2 & $350^{\prime}$ & 25.84 & $3.963 E+03$ & N.D. & 3.24 & $<0.001$ & $<0.001$ & $<0.001$ & $<0.001$ \\
\hline$M B C \cap B-2$ & $350^{\prime}$ & 25.74 & $4.610 \mathrm{E}+03$ & N.D. & 3.14 & $<0.001$ & $<0.001$ & & \\
\hline MBCSB-2 & $360^{\prime}$ & 25.91 & $5.740 \mathrm{E}+03$ & $1.594 \mathrm{E}+03$ & 3.31 & $<0.001$ & $<0.001$ & $<0.001$ & $<0.001$ \\
\hline MBCSB-2 & $360^{\prime}$ & 26.03 & $5.270 E+03$ & N.D. & 3.43 & $<0.001$ & $<0.001$ & & \\
\hline
\end{tabular}




\begin{tabular}{|c|c|c|c|c|c|c|c|c|c|}
\hline Well ID & $\begin{array}{l}\text { Depth } \\
\text { feet }\end{array}$ & $\begin{array}{c}\text { Vial } \\
\text { Mass } \\
8 \\
\end{array}$ & $\begin{array}{c}\mathrm{TCE} \\
\text { counts }\end{array}$ & $\begin{array}{r}\text { PCE } \\
\text { counts }\end{array}$ & $\begin{array}{c}\text { Sediment } \\
\text { Mass } \\
g \\
\end{array}$ & $\begin{array}{l}\text { TCE } \\
\text { conc. } \\
\text { ug/g } / g \\
\end{array}$ & $\begin{array}{l}\text { PCE } \\
\text { conc. } \\
\text { ug/g } \\
\end{array}$ & $\begin{array}{c}\text { TCE } \\
\text { avg. conc. } \\
\text { ug/g } \\
\end{array}$ & $\begin{array}{c}\text { PCE } \\
\text { avg. conc. } \\
\text { ug/g } \\
\end{array}$ \\
\hline $\mathrm{MBCSB}-3$ & $200^{\prime}$ & 25.61 & $6.483 E+05$ & $7.031 \mathrm{E}+03$ & 3.01 & 0.015 & $<0.001$ & 0.015 & $<0.001$ \\
\hline MBCSB-3 & $200^{\prime}$ & 25.72 & $6.910 E+05$ & $7.211 \mathrm{E}+03$ & 3.12 & 0.015 & $<0.001$ & & \\
\hline MBCSB-3 & $210^{\prime}$ & 25.63 & $2.627 \mathrm{E}+05$ & $2.756 \mathrm{E}+03$ & 3.03 & 0.006 & $<0.001$ & 0.007 & $<0.001$ \\
\hline MBCSB-3 & $210^{\prime}$ & 25.25 & $3.032 \mathrm{E}+05$ & $3.626 E+03$ & 2.65 & 0.008 & $<0.001$ & & \\
\hline MBCSB-3 & $220^{\prime}$ & 26.24 & $3.340 \mathrm{E}+05$ & $3.405 \mathrm{E}+03$ & 3.64 & 0.006 & $<0.001$ & 0.006 & $<0.001$ \\
\hline MBCSB-3 & $220^{\circ}$ & 26.50 & $3.495 E+05$ & $3.872 E+03$ & 3.90 & 0.006 & $<0.001$ & & \\
\hline MBCSB-3 & $230^{\prime}$ & 25.36 & $1.302 E+05$ & $1.632 E+03$ & 2.76 & 0.003 & $<0.001$ & 0.003 & $<0.001$ \\
\hline MBCSB-3 & $230^{\prime}$ & 24.63 & $6.394 E+04$ & $1.392 \mathrm{E}+03$ & 2.03 & 0.002 & $<0.001$ & & \\
\hline MBCSB-3 & $240^{\prime}$ & 25.97 & $1.930 \mathrm{E}+05$ & $2.069 E+03$ & 3.37 & 0.004 & $<0.001$ & 0.003 & $<0.001$ \\
\hline MBCSB-3 & $240^{\prime}$ & 25.07 & $9.929 \mathrm{E}+04$ & $1.425 E+03$ & 2.47 & 0.003 & $<0.001$ & & \\
\hline MBCSB-3 & $250^{\prime}$ & 25.92 & $1.991 E+06$ & $5.196 E+03$ & 3.32 & 0.041 & $<0.001$ & 0.051 & $<0.001$ \\
\hline MBCSB-3 & $250^{\prime}$ & 26.62 & $3.545 \mathrm{E}+06$ & $8.134 E+03$ & 4.02 & 0.060 & $<0.001$ & & \\
\hline MBCSB-3 & $260^{\prime}$ & 25.10 & $4.964 E+05$ & $2.136 \mathrm{E}+03$ & 2.50 & 0.014 & $<0.001$ & 0.011 & $<0.001$ \\
\hline MBCSB-3 & $260^{\prime}$ & 24.77 & $2.549 E+05$ & $1.324 E+03$ & 2.17 & 0.008 & $<0.001$ & & \\
\hline MBCSB-3 & $270^{\prime}$ & 25.44 & $4.096 E+05$ & $3.930 E+03$ & 2.84 & 0.010 & $<0.001$ & 0.009 & $<0.001$ \\
\hline MBCSB-3 & $270^{\prime}$ & 25.44 & $3.338 \mathrm{E}+05$ & $2.650 \mathrm{E}+03$ & 2.84 & 0.008 & $<0.001$ & & \\
\hline
\end{tabular}




\begin{tabular}{|c|c|c|c|c|c|c|c|c|c|}
\hline Well ID & $\begin{array}{l}\text { Depth } \\
\text { feet }\end{array}$ & $\begin{array}{c}\text { Vial } \\
\text { Mass } \\
g \\
\end{array}$ & $\begin{array}{l}\text { TCE } \\
\text { counts }\end{array}$ & $\begin{array}{c}\text { PCE } \\
\text { counts }\end{array}$ & $\begin{array}{c}\text { Sediment } \\
\text { Mass } \\
\mathrm{B} \\
\end{array}$ & $\begin{array}{l}\text { TCE } \\
\text { conc. } \\
\text { ug/g } \\
\end{array}$ & $\begin{array}{l}\mathrm{PCE} \\
\text { conc. } \\
\mathrm{ug} / \mathrm{g} \\
\end{array}$ & $\begin{array}{c}\text { TCE } \\
\text { avg. conc. } \\
\text { ug/g } \\
\end{array}$ & $\begin{array}{c}\text { PCE } \\
\text { avg. conc. } \\
\text { ug/g } \\
\end{array}$ \\
\hline MBCSB-4 & $200^{\prime}$ & 25.65 & $6.559 E+04$ & $1.153 E+04$ & 3.05 & 0.001 & $<0.001$ & $<0.001$ & $<0.001$ \\
\hline MBCSB-4 & $200^{\prime}$ & 26.01 & $2.966 \mathrm{E}+04$ & $5.551 E+03$ & 3.41 & $<0.001$ & $<0.001$ & & \\
\hline MBCSB-4 & $210^{\prime}$ & 26.00 & $2.506 E+04$ & $1.174 E+05$ & 3.40 & $<0.001$ & $<0.001$ & $<0.001$ & $<0.001$ \\
\hline MBCSB-4 & $210^{\prime}$ & 25.89 & $2.704 E+04$ & $1.318 E+05$ & 3.29 & $<0.001$ & $<0.001$ & & \\
\hline $\mathrm{MBCSB}-4$ & $220^{\prime}$ & 25.34 & $4.242 E+04$ & $1.349 E+0.4$ & 2.74 & $<0.001$ & $<0.001$ & 0.001 & $<0.001$ \\
\hline MBCSB-4 & $220^{\prime}$ & 26.15 & $7.630 \mathrm{E}+04$ & $2.545 E+04$ & 3.55 & 0.001 & $<0.001$ & & \\
\hline MBCSB-4 & $230^{\prime}$ & 25.45 & $9.691 E+05$ & $8.861 E+04$ & 2.85 & 0.020 & $<0.001$ & 0.024 & $<0.001$ \\
\hline MBCSB-4 & $230^{\circ}$ & 25.58 & $1.408 E+06$ & $1.852 E+05$ & 2.98 & 0.028 & 0.001 & & \\
\hline $\mathrm{MBCSB}-4$ & $240^{\prime}$ & 25.75 & $1.476 E+06$ & $4.600 E+05$ & 3.15 & 0.028 & 0.003 & 0.026 & 0.003 \\
\hline MBCSB-4 & $240^{\prime}$ & 25.97 & $1.389 E+06$ & $4.378 E+05$ & 3.37 & 0.025 & 0.003 & & \\
\hline MBCSB-4 & $250^{\prime}$ & 26.38 & $7.619 E+04$ & $1.040 \mathrm{E}+04$ & 3.78 . & 0.001 & $<0.001$ & 0.001 & $<0.001$ \\
\hline MBCSB-4 & $250^{\prime}$ & 26.33 & $6.091 E+04$ & $9.226 E+03$ & 3.73 & $<0.001$ & $<0.001$ & & \\
\hline $\mathrm{MBCSB}-4$ & $260^{\prime}$ & 25.73 & $1.757 \mathrm{E}+03$ & N.D. & 3.13 & $<0.001$ & $<0.001$ & $<0.001$ & $<0.001$ \\
\hline MBCSB-4 & $260^{\prime}$ & 25.42 & $2.146 \mathrm{E}+03$ & $1.336 E+03$ & 2.82 & $<0.001$ & $<0.001$ & & \\
\hline $\mathrm{MBCSB}-4$ & $270^{\prime}$ & 26.15 & N.D. & N.D. & 3.55 & $<0.001$ & $<0.001$ & $<0.001$ & $<0.001$ \\
\hline MBCSB-4 & $270^{\prime}$ & 26.26 & N.D. & N.D. & 3.66 & $<0.001$ & $<0.001$ & & \\
\hline MBCSB-4 & $280^{\prime}$ & 26.10 & N.D. & N.D. & 3.50 & $<0.001$ & $<0.001$ & $<0.001$ & $<0.001$ \\
\hline MBCSB-4 & $280^{\prime}$ & 26.52 & $1.765 E+03$ & N.D. & 3.92 & $<0.001$ & $<0.001$ & & \\
\hline MBCSB-4 & $290^{\prime}$ & 26.79 & $3.608 E+03$ & $1.445 E+03$ & 4.19 & $<0.001$ & $<0.001$ & $<0.001$ & $<0.001$ \\
\hline $\mathrm{MBCSB}-4$ & $290^{\prime}$ & 26.72 & $3.038 E+03$ & N.D. & 4.12 & $<0.001$ & $<0.001$ & & \\
\hline MBCSB-4 & $300^{\prime}$ & 25.83 & N.D. & N.D. & 3.23 & $<0.001$ & $<0.001$ & $<0.001$ & $<0.001$ \\
\hline $\mathrm{MBCSB}-4$ & $300^{\prime}$ & 26.00 & N.D. & $1.310 \mathrm{E}+03$ & 3.40 & $<0.001$ & $<0.001$ & & \\
\hline MBCSB-4 & $310^{\prime}$ & 25.36 & $7.219 E+04$ & $3.099 E+04$ & 2.76 & 0.002 & $<0.001$ & 0.002 & $<0.001$ \\
\hline MBCSB-4 & $310^{\prime}$ & 25.65 & $1.250 \mathrm{E}+05$ & $5.542 E+04$ & 3.05 & 0.002 & $<0.001$ & & \\
\hline MBCSB-4 & $320^{\prime}$ & 25.95 & $1.394 E+05$ & $1.893 E+06$ & 3.35 & 0.002 & 0.011 & 0.003 & 0.013 \\
\hline MBCSB-4 & $320^{\prime}$ & 26.07 & $1.848 \mathrm{E}+05$ & $2.402 E+06$ & 3.47 & 0.003 & 0.014 & & \\
\hline $\mathrm{MBCSB}-4$ & $330^{\prime}$ & 25.43 & $1.232 E+05$ & $2.152 E+06$ & 2.83 & 0.003 & 0.015 & 0.003 & 0.016 \\
\hline $\mathrm{MBCSB}-4$ & $330^{\prime}$ & 25.61 & $1.365 E+05$ & $2.373 E+06$ & 3.01 & 0.003 & 0.016 & & \\
\hline MBCSB-4 & $340^{\prime}$ & 25.88 & $1.893 E+06$ & $4.294 E+06$ & 3.28 & 0.035 & 0.027 & 0.040 & 0.030 \\
\hline MBCSB-4 & $340^{\prime}$ & 25.37 & $2.082 E+06$ & $4.645 E+06$ & 2.77 & 0.045 & 0.034 & & \\
\hline MBCSB-4 & $350^{\prime}$ & 25.47 & $2.292 E+06$ & $3.725 E+06$ & 2.87 & 0.048 & 0.026 & 0.054 & 0.030 \\
\hline MBCSB-4 & $350^{\prime}$ & 25.35 & $2.798 E+06$ & $4.590 E+06$ & 2.75 & 0.061 & 0.034 & & \\
\hline MBCSB-4 & $360^{\prime}$ & 25.57 & $4.691 \mathrm{E}+06$ & $1.546 E+06$ & 2.97 & 0.095 & 0.011 & 0.094 & 0.011 \\
\hline MBCSB-4 & $360^{\prime}$ & 25.68 & $4.813 E+06$ & $1.611 E+06$ & 3.08 . & 0.094 & 0.011 & & \\
\hline
\end{tabular}




\begin{tabular}{|c|c|c|c|c|c|c|c|c|c|}
\hline Well ID & $\begin{array}{l}\text { Depth } \\
\text { feet }\end{array}$ & $\begin{array}{c}\text { Vial } \\
\text { Mass } \\
8 \\
\end{array}$ & $\begin{array}{l}\text { TCE } \\
\text { counts }\end{array}$ & $\begin{array}{c}\text { PCE } \\
\text { counts }\end{array}$ & $\begin{array}{c}\text { Sediment } \\
\text { Mass } \\
? \\
\end{array}$ & $\begin{array}{l}\text { TCE } \\
\text { cone. } \\
\text { ug/g } \\
\end{array}$ & $\begin{array}{l}\mathrm{PCE} \\
\text { conc. } \\
u g / g \\
\end{array}$ & $\begin{array}{c}\text { TCE } \\
\text { avg. conc. } \\
\text { ug/g } \\
\end{array}$ & $\begin{array}{c}\text { PCE } \\
\text { avg. conc. } \\
\text { ug/g }\end{array}$ \\
\hline MBCSB-5 & $200^{\prime}$ & 25.70 & $1.217 \mathrm{E}+05$ & $1.785 E+04$ & 3.10 & 0.003 & $<0.001$ & 0.003 & $<0.001$ \\
\hline MBCSB-5 & $200^{\prime}$ & 25.42 & $1.015 E+05$ & $1.493 E+04$ & 2.82 & 0.003 & $<0.001$ & & \\
\hline MBCSB-5 & $210^{\circ}$ & 25.54 & $6.335 E+03$ & $1.855 E+03$ & 2.94 & $<0.001$ & $<0.001$ & $<0.001$ & $<0.001$ \\
\hline MBCSB-5 & $210^{\prime}$ & 25.29 & $9.356 E+03$ & $3.534 \mathrm{E}+03$ & 2.69 & $<0.001$ & $<0.001$ & & \\
\hline MBCSB-5 & $220^{\prime}$ & 27.02 & $2.809 E+04$ & $1.868 \mathrm{E}+03$ & 4.42 & $<0.001$ & $<0.001$ & $<0.001$ & $<0.001$ \\
\hline MBCSB-5 & $220^{\prime}$ & 26.21 & $1.816 E+04$ & $1.925 \mathrm{E}+03$ & 3.61 & $<0.001$ & $<0.001$ & & \\
\hline MBCSB-5 & $230^{\prime}$ & 25.83 & N.D. & $1.416 \mathrm{E}+03$ & 3.23 & $<0.001$ & $<0.001$ & $<0.001$ & $<0.001$ \\
\hline MBCSB-5 & $230^{\circ}$ & 25.88 & $1.768 E+03$ & $2.432 \mathrm{E}+03$ & 3.28 & $<0.001$ & $<0.001$ & & \\
\hline MBCSB-5 & $240^{\prime}$ & 24.42 & N.D. & N.D. & 1.82 & $<0.001$ & $<0.001$ & $<0.001$ & $<0.001$ \\
\hline MBCSB-5 & $240^{\prime}$ & 23.85 & N.D. & N.D. & 1.25 & $<0.001$ & $<0.001$ & & \\
\hline MBCSB-5 & $250^{\circ}$ & 27.44 & $1.060 \mathrm{E}+06$ & $1.606 \mathrm{E}+05$ & 4.84 & 0.018 & $<0.001$ & 0.013 & $<0.001$ \\
\hline MBCSB-5 & $250^{\prime}$ & 28.11 & $4.828 E+05$ & $5.197 E+04$ & 5.51 & 0.007 & $<0.001$ & & \\
\hline MBCSB-5 & $260^{\prime}$ & 28.22 & $1.095 E+06$ & $3.554 E+04$ & 5.62 & 0.016 & $<0.001$ & 0.017 & $<0.001$ \\
\hline MBCSB-5 & $260^{\prime}$ & 28.00 & $1 .: 37 E+06$ & $3.098 E+04$ & 5.40 & 0.017 & $<0.001$ & & \\
\hline MBCSB-5 & $270^{\prime}$ & 25.44 & $2.276 E+03$ & N.D. & 2.84 & $<0.001$ & $<0.001$ & $<0.001$ & $<0.001$ \\
\hline MBCSB-5 & $270^{\prime}$ & 25.61 & $2.525 E+03$ & N.D. & 3.01 & $<0.001$ & $<0.001$ & & \\
\hline MBCSB-5 & $280^{\circ}$ & 25.70 & N.D. & $1.517 E+03$ & 3.10 & $<0.001$ & $<0.001$ & $<0.001$ & $<0.001$ \\
\hline MBCSB-5 & $280^{\prime}$ & 26.66 & N.D. & $9.750 E+03$ & 4.06 & $<0.001$ & $<0.001$ & & \\
\hline MBCSB-5 & $290^{\prime}$ & 26.02 & N.D. & $1.370 E+03$ & 3.42 & $<0.001$ & $<0.001$ & $<0.001$ & $<0.001$ \\
\hline MBCSB-5 & $290^{\prime}$ & 26.79 & N.D. & N.D. & 4.19 & $<0.001$ & $<0.001$ & & \\
\hline MBCSB-5 & $300^{\prime}$ & 26.81 & N.D. & $5.027 E+04$ & 4.21 & $<0.001$ & $<0.001$ & $<0.001$ & $<0.001$ \\
\hline MBCSB-5 & $300^{\prime}$ & 26.77 & N.D. & N.D. & 4.17 & $<0.001$ & $<0.001$ & & \\
\hline MBCSB-5 & $310^{\prime}$ & 26.61 & $7.312 E+03$ & $3.703 E+04$ & 4.01 & $<0.001$ & $<0.001$ & $<0.001$ & $<0.001$ \\
\hline MBCSB-5 & $310^{\prime}$ & 27.30 & $7.628 \mathrm{E}+03$ & $3.851 E+04$ & 4.70 & $<0.001$ & $<0.001$ & & \\
\hline MBCSB-5 & $320^{\prime}$ & 26.06 & $9.853 \mathrm{E}+03$ & $4.542 E+04$ & 3.46 & $<0.001$ & $<0.001$ & $<0.001$ & $<0.001$ \\
\hline MBCSB-5 & $320^{\prime}$ & 25.68 & $1.466 \mathrm{E}+04$ & $1.077 \mathrm{E}+05$ & 3.08 & $<0.001$ & $<0.001$ & & \\
\hline MBCSB-5 & $330^{\prime}$ & 26.58 & $2.192 \mathrm{E}+03$ & $1.554 E+03$ & 3.98 & $<0.001$ & $<0.001$ & $<0.001$ & $<0.001$ \\
\hline MBCSB-5 & $330^{\prime}$ & 26.27 & $2.283 E+03$ & $1.654 E+03$ & 3.67 & $<0.001$ & $<0.001$ & & \\
\hline MBCSB-5 & $340^{\prime}$ & 25.85 & $1.646 \mathrm{E}+04$ & $1.027 E+05$ & 3.25 & $<0.001$ & $<0.001$ & $<0.001$ & $<0.001$ \\
\hline MBCSB-5 & $340^{\prime}$ & 25.91 & $1.629 E+04$ & $4.676 E+04$ & 3.31 & $<0.001$ & $<0.001$ & & \\
\hline MBCSB-5 & $350^{\circ}$ & 25.91 & $7.867 E+0 S$ & $4.755 E+05$ & 3.31 & 0.020 & 0.003 & 0.020 & 0.004 \\
\hline MBCSB-5 & $350^{\circ}$ & 26.22 & $9.409 \mathrm{E}+05$ & $6.699 E+05$ & 3.62 & 0.021 & 0.004 & & \\
\hline MBCSB-5 & $360^{\prime}$ & 25.87 & $1.939 E+06$ & $2.032 E+06$ & 3.27 & 0.049 & 0.015 & 0.046 & 0.013 \\
\hline MBCSB-5 & $360^{\circ}$ & 25.90 & $1.734 E+06$ & $1.582 E+06$ & 3.30 & 0.043 & 0.011 & & \\
\hline MBCSB-5 & $365^{\prime}$ & 25.91 & $2.834 E+05$ & $9.472 \mathrm{E}+05$ & 3.31 & 0.007 & 0.007 & 0.015 & 0.007 \\
\hline MBCSB-5 & $365^{\prime}$ & 27.03 & $1.199 \mathrm{E}+06$ & $1.374 E+06$ & 4.43 & 0.022 & 0.007 & & \\
\hline
\end{tabular}




\begin{tabular}{|c|c|c|c|c|c|c|c|c|c|}
\hline Well ID & $\begin{array}{c}\text { Depth } \\
\text { feet }\end{array}$ & $\begin{array}{c}\text { Vial } \\
\text { Mass } \\
8 \\
\end{array}$ & $\begin{array}{l}\text { TCE } \\
\text { counts }\end{array}$ & $\begin{array}{c}\text { PCE } \\
\text { counts }\end{array}$ & $\begin{array}{c}\text { Sediment } \\
\text { Mass } \\
g \\
\end{array}$ & $\begin{array}{l}\text { TCE } \\
\text { conc. } \\
\text { ug/g }\end{array}$ & $\begin{array}{l}\text { PCE } \\
\text { conc. } \\
\text { ug/g } \\
\end{array}$ & 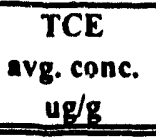 & $\begin{array}{c}\text { PCE } \\
\text { avg. conc. } \\
\text { ug/g } \\
\end{array}$ \\
\hline MBCSB-6 & $200^{\prime}$ & 25.70 & $2.301 E+03$ & $2.539 E+03$ & 3.10 & $<0.001$ & $<0.001$ & $<0.001$ & $<0.001$ \\
\hline MBCSB-6 & $200^{\prime}$ & 26.43 & $2.596 \mathrm{E}+03$ & $2.097 E+03$ & 3.83 & $<0.001$ & $<0.001$ & & \\
\hline $\mathrm{MBCSB}-6$ & $210^{\prime}$ & 28.98 & $1.318 \mathrm{E}+04$ & $2.150 E+03$ & 6.38 & $<0.001$ & $<0.001$ & $<0.001$ & $<0.001$ \\
\hline MBCSB- 6 & $210^{\prime}$ & 28.45 & $6.583 E+03$ & $1.957 \mathrm{E}+03$ & 5.85 & $<0.001$ & $<0.001$ & & \\
\hline MBCSB-6 & $220^{\prime}$ & 27.00 & $2.648 E+03$ & $1.585 E+03$ & 4.40 & $<0.001$ & $<0.001$ & $<0.001$ & $<0.001$ \\
\hline MBCSB-6 & $220^{\prime}$ & 27.24 & $3.324 E+03$ & $1.712 \mathrm{E}+03$ & 4.64 & $<0.001$ & $<0.001$ & & \\
\hline MBCSB-6 & $230^{\prime}$ & 25.97 & N.D. & N.D. & 3.37 & $<0.001$ & $<0.001$ & $<0.001$ & $<0.001$ \\
\hline MBCSB-6 & $230^{\prime}$ & 26.22 & N.D. & N.D. & 3.62 & $<0.001$ & $<0.001$ & & \\
\hline MBCSB-6 & $240^{\prime}$ & 26.17 & N.D. & N.D. & 3.57 & $<0.001$ & $<0.001$ & $<0.001$ & $<0.001$ \\
\hline MBCSB-6 & $240^{\prime}$ & 25.91 & N.D. & N.D. & 3.31 & $<0.001$ & $<0.001$ & & \\
\hline MBCSB-6 & $250^{\prime}$ & 26.26 & N.D. & N.D. & 3.66 & $<0.001$ & $<0.001$ & $<0.001$ & $<0.001$ \\
\hline MBCSB-6 & $250^{\prime}$ & 27.01 & N.D. & N.D. & 4.41 & $<0.001$ & $<0.001$ & & \\
\hline MBCSB-6 & $260^{\prime}$ & 26.21 & N.D. & N.D. & 3.61 & $<0.001$ & $<0.001$ & $<0.001$ & $<0.001$ \\
\hline MBCSB-6 & $260^{\circ}$ & 27.20 & N.D. & N.D. & 4.60 & $<0.001$ & $<0.001$ & & \\
\hline MBCSB-6 & $270^{\prime}$ & 26.32 & N.D. & N.D. & 3.72 & $<0.001$ & $<0.001$ & $<0.001$ & $<0.001$ \\
\hline MBCSB-6 & $270^{\prime}$ & 26.39 & N.D. & N.D. & 3.79 & $<0.001$ & $<0.001$ & & \\
\hline MBCSB-6 & $280^{\prime}$ & 26.39 & N.D. & N.D. & 3.79 & $<0.001$ & $<0.001$ & $<0.001$ & $<0.001$ \\
\hline MBCSB-6 & $280^{\prime}$ & 26.36 & N.D. & N.D. & 3.76 & $<0.001$ & $<0.001$ & & \\
\hline MBCSB-6 & $290^{\prime}$ & 26.35 & N.D. & N.D. & 3.75 & $<0.001$ & $<0.001$ & $<0.001$ & $<0.001$ \\
\hline MBCSB-6 & $290^{\prime}$ & 25.83 & N.D. & N.D. & 3.23 & $<0.001$ & $<0.001$ & & \\
\hline MBCSB-6 & $300^{\prime}$ & 26.60 & N.D. & N.D. & 4.00 & $<0.001$ & $<0.001$ & $<0.001$ & $<0.001$ \\
\hline MBCSB-6 & $300^{\prime}$ & 26.03 & N.D. & N.D. & 3.43 & $<0.001$ & $<0.001$ & & \\
\hline MBCSB-6 & $310^{\prime}$ & 26.19 & N.D. & N.D. & 3.59 & $<0.001$ & $<0.001$ & $<0.001$ & $<0.001$ \\
\hline MBCSB-6 & $310^{\prime}$ & 26.08 & N.D. & N.D. & 3.48 & $<0.001$ & $<0.001$ & & \\
\hline MBCSB-6 & $320^{\prime}$ & 26.03 & N.D. & $1.426 E+03$ & 3.43 & $<0.001$ & $<0.001$ & $<0.001$ & $<0.001$ \\
\hline MBCSB-6 & $320^{\prime}$ & 26.53 & N.D. & N.D. & 3.93 & $<0.001$ & $<0.001$ & & \\
\hline MBCSB-6 & $330^{\prime}$ & 25.11 & N.D. & N.D. & 2.51 & $<0.001$ & $<0.001$ & $<0.001$ & $<0.001$ \\
\hline MBCSB-6 & $330^{\prime}$ & 25.24 & N.D. & N.D. & 2.64 & $<0.001$ & $<0.001$ & & \\
\hline MBCSB-6 & $340^{\prime}$ & 25.43 & N.D. & N.D. & 2.83 & $<0.001$ & $<0.001$ & $<0.001$ & $<0.001$ \\
\hline MBCSB-6 & $340^{\prime}$ & 26.90 & N.D. & N.D. & 4.30 & $<0.001$ & $<0.001$ & & \\
\hline MBCSB-6 & $350^{\prime}$ & 25.97 & N.D. & N.D. & 3.37 & $<0.001$ & $<0.001$ & $<0.001$ & $<0.001$ \\
\hline MBCSB-6 & $350^{\circ}$ & 26.45 & N.D. & N.D. & 3.85 & $<0.001$ & $<0.001$ & & \\
\hline MBCSB-6 & $360^{\prime}$ & 26.78 & N.D. & N.D. & 4.18 & $<0.001$ & $<0.001$ & $<0.001$ & $<0.001$ \\
\hline MBCSB-6 & $360^{\prime}$ & 26.50 & N.D. & N.D. & 3.90 & $<0.001$ & $<0.001$ & & \\
\hline
\end{tabular}




\begin{tabular}{|c|c|c|c|c|c|c|c|c|c|}
\hline Well ID & $\begin{array}{l}\text { Depth } \\
\text { feet }\end{array}$ & $\begin{array}{c}\text { Vial } \\
\text { Mass } \\
8 \\
\end{array}$ & $\begin{array}{c}\text { TCE } \\
\text { counts }\end{array}$ & $\begin{array}{c}\text { PCE } \\
\text { counts }\end{array}$ & $\begin{array}{c}\text { Sediment } \\
\text { Mass } \\
8 \\
\end{array}$ & $\begin{array}{l}\text { TCE } \\
\text { conc. } \\
\text { ug/g } \\
\end{array}$ & $\begin{array}{l}\mathrm{PCE} \\
\text { conc. } \\
\mathrm{ug} / \mathrm{g} \\
\end{array}$ & $\begin{array}{r}\text { TCE } \\
\text { avg. conc. } \\
\text { ug/g } \\
\end{array}$ & $\begin{array}{c}\mathrm{PCE} \\
\text { avg. conc. } \\
\text { ug/g } \\
\end{array}$ \\
\hline MBCSB-7 & $200^{\prime}$ & 25.56 & $2.328 \mathrm{E}+03$ & $2.334 E+03$ & 2.96 & $<0.001$ & $<0.001$ & $<0.001$ & $<0.001$ \\
\hline MBCSB-7 & $200^{\prime}$ & 26.20 & $1.721 E+03$ & $1.548 \mathrm{E}+03$ & 3.60 & $<0.001$ & $<0.001$ & & \\
\hline MBCSB-7 & $205^{\prime}$ & 25.55 & N.D. & N.D. & 2.95 & $<0.001$ & $<0.001$ & $<0.001$ & $<0.001$ \\
\hline MBCSB-7 & $205^{\prime}$ & 25.19 & N.D. & N.D. & 2.59 & $<0.001$ & $<0.001$ & & \\
\hline MBCSB-7 & $210^{\prime}$ & 25.58 & $1.549 \mathrm{E}+03$ & N.D. & 2.98 & $<0.001$ & $<0.001$ & $<0.001$ & $<0.001$ \\
\hline MBCSB-7 & $210^{\circ}$ & 25.80 & $2.170 \mathrm{E}+03$ & N.D. & 3.20 & $<0.001$ & $<0.001$ & & \\
\hline MBCSB-7 & $220^{\prime}$ & 26.07 & N.D. & N.D. & 3.47 & $<0.001$ & $<0.001$ & $<0.001$ & $<0.001$ \\
\hline MBCSB-7 & $220^{\circ}$ & 25.82 & N.D. & N.D. & 3.22 & $<0.001$ & $<0.001$ & & \\
\hline MBCSB-7 & $230^{\prime}$ & 26.64 & $1.496 E+03$ & N.D. & 4.04 & $<0.001$ & $<0.001$ & $<0.001$ & $<0.001$ \\
\hline MBCSB-7 & $230^{\prime}$ & 25.61 & $2.153 E+03$ & N.D. & 3.01 & $<0.001$ & $<0.001$ & & \\
\hline MBCSB-7 & $240^{\circ}$ & 25.67 & $2.104 E+03$ & N.D. & 3.07 & $<0.001$ & $<0.001$ & $<0.001$ & $<0.001$ \\
\hline MBCSB-7 & $240^{\prime}$ & 25.74 & $1.900 \mathrm{E}+03$ & N.D. & 3.14 & $<0.001$ & $<0.001$ & & \\
\hline MBCSB-7 & $250^{\prime}$ & 25.44 & $1.654 \mathrm{E}+03$ & N.D. & 2.84 & $<0.001$ & $<0.001$ & $<0.001$ & $<0.001$ \\
\hline MBCSB-7 & $250^{\circ}$ & 26.25 & $1.709 E+03$ & N.D. & 3.65 & $<0.001$ & $<0.001$ & & \\
\hline MBCSB-7 & $260^{\prime}$ & 27.03 & N.D. & N.D. & 4.43 & $<0.001$ & $<0.001$ & $<0.001$ & $<0.001$ \\
\hline MBCSB-7 & $260^{\prime}$ & 26.65 & N.D. & N.D. & 4.05 & $<0.001$ & $<0.001$ & & \\
\hline MBCSB-7 & $270^{\prime}$ & 25.93 & $2.057 E+03$ & $1.375 E+03$ & 3.33 & $<0.001$ & $<0.001$ & $<0.001$ & $<0.001$ \\
\hline MBCSB-7 & $270^{\prime}$ & 25.98 & $2.477 E+03$ & $1.753 E+03$ & 3.38 & $<0.001$ & $<0.001$ & & \\
\hline MBCSB-7 & $280^{\prime}$ & 26.75 & N.D. & N.D. & 4.15 & $<0.001$ & $<0.001$ & $<0.001$ & $<0.001$ \\
\hline MBCSB-7 & $280^{\prime}$ & 26.14 & N.D. & N.D. & 3.54 & $<0.001$ & $<0.001$ & & \\
\hline MBCSB-7 & $290^{\prime}$ & 26.21 & N.D. & N.D. & 3.61 & $<0.001$ & $<0.001$ & $<0.001$ & $<0.001$ \\
\hline MBCSB-7 & $290^{\circ}$ & 25.88 & N.D. & N.D. & 3.28 & $<0.001$ & $<0.001$ & & \\
\hline MBCSB-7 & $300^{\prime}$ & 26.07 & $1.786 E+03$ & N.D. & 3.47 & $<0.001$ & $<0.001$ & $<0.001$ & $<0.001$ \\
\hline MBCSB-7 & $300^{\prime}$ & 26.26 & N.D. & $2.992 E+03$ & 3.66 & $<0.001$ & $<0.001$ & & \\
\hline MBCSB-7 & $310^{\circ}$ & 26.21 & N.D. & N.D. & 3.61 & $<0.001$ & $<0.001$ & $<0.001$ & $<0.001$ \\
\hline MBCSB-7 & $310^{\prime}$ & 26.37 & N.D. & N.D. & 3.77 & $<0.001$ & $<0.001$ & & \\
\hline MBCSB-7 & $320^{\prime}$ & 25.55 & N.D. & N.D. & 2.95 & $<0.001$ & $<0.001$ & $<0.001$ & $<0.001$ \\
\hline MBCSB-7 & $320^{\prime}$ & 25.92 & N.D. & N.D. & 3.32 & $<0.001$ & $<0.001$ & & \\
\hline MBCSB-7 & $330^{\prime}$ & 26.05 & $2.213 E+03$ & N.D. & 3.45 & $<0.001$ & $<0.001$ & $<0.001$ & $<0.001$ \\
\hline MBCSB-7 & $330^{\circ}$ & 25.78 & $2.088 E+03$ & N.D. & 3.18 & $<0.001$ & $<0.001$ & & \\
\hline MBCSB-7 & $340^{\circ}$ & 25.53 & N.D. & N.D. & 2.93 & $<0.001$ & $<0.001$ & $<0.001$ & $<0.001$ \\
\hline MBCSB-7 & $340^{\circ}$ & 25.67 & N.D. & N.D. & 3.07 & $<0.001$ & $<0.001$ & & \\
\hline MBCSB-7 & $350^{\prime}$ & 26.20 & N.D. & N.D. & 3.60 & $<0.001$ & $<0.001$ & $<0.001$ & $<0.001$ \\
\hline MBCSB-7 & $350^{\circ}$ & 26.49 & N.D. & N.D. & 3.89 & $<0.001$ & $<0.001$ & & \\
\hline MBCSB-7 & $360^{\circ}$ & 25.47 & N.D. & N.D. & 2.87 & $<0.001$ & $<0.001$ & $<0.001$ & $<0.001$ \\
\hline MBCSB-7 & $360^{\prime}$ & 25.69 & N.D. & N.D. & 3.09 & $<0.001$ & $<0.001$ & & \\
\hline MBCSB-7 & $365^{\prime}$ & 26.07 & N.D. & N.D. & 3.47 & $<0.001$ & $<0.001$ & $<0.001$ & $<0.001$ \\
\hline MBCSB-7 & $365^{\prime}$ & 25.74 & N.D. & N.D. & 3.14 & $<0.001$ & $<0.001$ & & \\
\hline
\end{tabular}


Attachment B - Raw Data from the Purge and Trap (MicroSeeps Ltd) Analysis 
.... SAVANKAH RIVER SITE .....

.... CRETACEOUS aqUIFER STUDY .....

..... A/M AREA WELL CORE SAMPLES .....

..... DYHASOILS PURGE \& TRAP .....

\begin{tabular}{|c|c|c|c|c|c|c|c|c|c|}
\hline $\begin{array}{l}\text { SAMPLE } \\
\text { WAME }\end{array}$ & $\begin{array}{r}\text { VINYL } \\
\text { CHLORIDE } \\
(U g / g)\end{array}$ & $\begin{array}{r}\text { METHYLENE } \\
\text { CHLORIDE } \\
(n g / g)\end{array}$ & $\begin{array}{r}\text { TRANS } 1,2 \\
\text { DICHLORO } \\
\text { ETHYLEME } \\
(\mathrm{ng} / \mathrm{g})\end{array}$ & $\begin{array}{r}\text { CHLORO } \\
\text { FORM } \\
\text { (ng/g) }\end{array}$ & $\begin{array}{l}\text { III TRI } \\
\text { CHLORO } \\
\text { ETHANE } \\
\text { (ng/g) }\end{array}$ & $\begin{array}{r}\text { CARBOW } \\
\text { TETRA } \\
\text { CHLORIDE } \\
\text { (ng/g) }\end{array}$ & $\begin{array}{r}\text { TRI } \\
\text { CHLORO } \\
\text { ETHYLENE } \\
(n g / g)\end{array}$ & $\begin{array}{r}\text { TEYRA } \\
\text { CHLORO } \\
\text { ETHYLENE } \\
(\mathrm{ng} / \mathrm{g})\end{array}$ & $\begin{array}{r}\text { FILE } \\
*\end{array}$ \\
\hline AB-SCB-1-1 & $<1$ & $<20$ & $<20$ & $<1$ & 25 & $<1$ & 8 & 2 & 12104 \\
\hline MB-SCB-1-2 & $<1$ & $<20$ & $<20$ & $<1$ & $<1$ & $<1$ & $<1$ & $<1$ & 12105 \\
\hline MB-SCB-1-3 & $<1$ & $<20$ & $<20$ & $<1$ & $<1$ & $<1$ & $<1$ & $<1$ & 12106 \\
\hline$M B-5 C B-1-4$ & $<1$ & $<20$ & $<20$ & $<1$ & $<1$ & $<9$ & $<1$ & $<1$ & T2 107 \\
\hline 148-SCB-1-5 & $<1$ & $<20$ & $<20$ & $<1$ & 14 & $<1$ & 5 & 1 & 12108 \\
\hline 198-sc8-1-6 & $<1$ & $<20$ & $<20$ & $<1$ & $<1$ & $<1$ & $<1$ & $<1$ & T2 109 \\
\hline$M B-5 C B-1-6 A$ & $<1$ & $<20$ & $<20$ & $<1$ & $<1$ & $<1$ & $<1$ & $<1$ & T2 110 \\
\hline MB-SCB-2-1 & $<1$ & $<20$ & $<20$ & $<1$ & $<1$ & $<1$ & $<1$ & $<1$ & 12111 \\
\hline$M B-5 C B-2-1 A$ & $<1$ & $<20$ & $<20$ & $<1$ & $<1$ & $<1$ & $<1$ & $<1$ & $T 2112$ \\
\hline$M B-5 C 8-2-2$ & $<1$ & $<20$ & $<20$ & $<1$ & $<1$ & $<1$ & $<1$ & $<1$ & 12113 \\
\hline MB-SCB-2-3 & $<1$ & $<20$ & $<20$ & $<1$ & $<1$ & $<1$ & $<1$ & $<1$ & 12. 114 \\
\hline MB-SCB-2-4 & $<1$ & $<20$ & $<20$ & $<1$ & $<1$ & $<1$ & $<1$ & $<1$ & 12118 \\
\hline MB-SCB-2-5 & $<1$ & $<20$ & $<20$ & $<1$ & $<1$ & $<1$ & $<1$ & $<1$ & $121: 9$ \\
\hline MB-SCB-2-6 & $<1$ & $<20$ & $<20$ & $<1$ & $<1$ & $<1$ & $<1$ & $<1$ & 12120 \\
\hline$M B-5 C B-2-7$ & $<1$ & $<20$ & $<20$ & $<1$ & $<1$ & $<1$ & $<1$ & $<1$ & T2 121 \\
\hline AB-SCB-2-8 & $<1$ & $<20$ & $<20$ & $<1$ & $<1$ & $<1$ & $<1$ & $<1$ & T2 122 \\
\hline$M B-\operatorname{scB}-2-9$ & $<1$ & $<20$ & $<20$ & $<1$ & $<1$ & $<1$ & $<1$ & $<1$ & 12123 \\
\hline$M B-\operatorname{sc} 8-2-10$ & $<1$ & $<20$ & $<20$ & $<1$ & $<1$ & $<1$ & $<1$ & $<1$ & 12124 \\
\hline MB-scB-2-11 & $<1$ & $<20$ & $<20$ & $<1$ & 14 & $<1$ & $<1$ & $<1$ & 12125 \\
\hline$M B-S C B-2-11 A$ & $<1$ & $<20$ & $<20$ & $<1$ & $<1$ & $<1$ & $<1$ & $<1$ & 12126 \\
\hline$M B-S C B-2-12$ & $<1$ & $<20$ & $<20$ & $<1$ & $<1$ & $<1$ & $<1$ & $<1$ & 12127 \\
\hline$M B-5 C B-2-13$ & $<1$ & $<20$ & $<20$ & $<1$ & $<1$ & $<1$ & $<1$ & $<1$ & 12128 \\
\hline AB-sc8-2- 14 & $<1$ & $<20$ & $<20$ & $<1$ & $<1$ & $<1$ & $<1$ & $<1$ & 12133 \\
\hline MB-SCB-2-15 & $<1$ & $<20$ & $<20$ & $<1$ & $<1$ & $<1$ & 1 & $<1$ & 12134 \\
\hline$M B-S C B-2-16$ & $<1$ & $<20$ & $<20$ & $<1$ & $<1$ & $<1$ & $<1$ & $<1$ & 12135 \\
\hline MB-SCB-4-1 & $<1$ & $<20$ & $<20$ & 4 & $<1$ & $<1$ & $<1$ & $<1$ & 12142 \\
\hline AB-SCB-4-1A & $<1$ & $<20$ & $<20$ & 3 & $<1$ & $<1$ & $<1$ & $<1$ & 12143 \\
\hline MB-SCB-4-2 & $<1$ & $<20$ & $<20$ & 2 & $<1$ & $<1$ & $<1$ & $<1$ & 12144 \\
\hline MB-SCB-4-3 & $<1$ & $<20$ & $<20$ & 2 & $<1$ & $<1$ & $<1$ & $<1$ & 12145 \\
\hline MB-SCB- $4-6$ & $<1$ & $<20$ & $<20$ & 2 & $<1$ & $<1$ & $<1$ & $<1$ & 12149 \\
\hline$M B-5 C B-4-8$ & $<1$ & $<20$ & $<20$ & $<1$ & $<1$ & $<1$ & $<1$ & $<1$ & T2 151 \\
\hline MB-SCE-4-9 & $<1$ & $<20$ & $<20$ & $<1$ & $<1$ & $<1$ & $<1$ & $<1$ & 12152 \\
\hline MB-SCB- $4-10$ & $<1$ & $<20$ & $<20$ & $<1$ & $<1$ & $<1$ & $<1$ & $<1$ & T2 153 \\
\hline$M B-5 C 8-4-11$ & $<1$ & $<20$ & $<20$ & $<1$ & $<1$ & $<1$ & $<1$ & $<1$ & 12154 \\
\hline$M B-5 C B-4-11 A$ & $<1$ & $<20$ & $<20$ & $<1$ & $<1$ & $<1$ & $<1$ & $<1$ & 12155 \\
\hline$M B-S C B-4-12$ & $<1$ & $<20$ & $<20$ & $<1$ & 1 & $<1$ & 1 & $<1$ & 12159 \\
\hline MB-SCB-4- 13 & $<1$ & $<20$ & $<20$ & $<1$ & 1 & $<1$ & 1 & 6 & T2 160 \\
\hline MB-SCB- $4-15$ & $<1$ & $<20$ & $<20$ & 1 & $<1$ & $<1$ & 42 & 15 & 12162 \\
\hline MB-SCB-4-17 & $<1$ & $<20$ & $<20$ & $<1$ & $<1$ & $<1$ & 78 & 4 & 12164 \\
\hline MB-SCB-1-SB1 & $<1$ & $<20$ & $<20$ & $<1$ & 26 & $<1$ & 9 & 2 & 12103 \\
\hline$M B-S C B-2-S B 1$ & $<1$ & $<20$ & $<20$ & $<1$ & $<1$ & $<1$ & $<1$ & $<1$ & 12115 \\
\hline$M B-S C B-2-S B 2$ & $<1$ & $<20$ & $<20$ & $<1$ & $<1$ & $<1$ & $<1$ & $<1$ & 12129 \\
\hline$M B-S C B-4-581$ & $<1$ & $<20$ & $<20$ & $<1$ & 1 & $<1$ & $<1$ & $<1$ & 12161 \\
\hline$M B-S C B-4-5 B 2$ & $<1$ & $<20$ & $<20$ & $<1$ & $<1$ & $<1$ & $<1$ & $<1$ & $T 2158$ \\
\hline
\end{tabular}


MICROSEEPS LTD.

CIIENT: SRS / John Hazlos

LOCATION: $M$ Area

PROJECP(1): AM Cretaclous Aquerfer

PAGE: of:

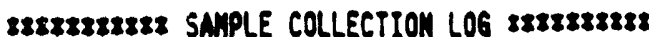

SAMPLER MAME(S): Kal

S.:L Boning MO SCB-1

\begin{tabular}{|c|c|c|c|c|c|c|c|c|c|c|c|}
\hline SAMPLE ID: & DATE & TInE & SEO. 1 & SAMPLE & SMMP & LE TY & & SMPLE & & & \\
\hline & & & & DEPTH & 6 & 51 & 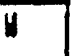 & SIZE & & & \\
\hline 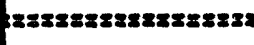 & & & 政 & & 232823\%: & & & & 232823289 & 32x38s: & \\
\hline MB.send SO.L & $7 / 27 / 43$ & $m$ & 1 & $3+80$ & & - & & 40 & 5.0 & 1120 & 5- sal sit \\
\hline M8.san.1-1 & & 1105 & 2 & $310^{\circ}$ & & & & & " & 11 & 5.0 Soil \\
\hline MB.SCA.1.2 & & 1122 & 3 & $320^{\circ}$ & & & & & " & $"$ & 5.0 \\
\hline$M P .3 C_{0.1 .3}$ & & 1140 & 4 & $330^{\circ}$ & & & & & $=$ & 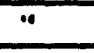 & 5.1 \\
\hline MB.SCB.1.4 & & 1155 & 5 & 340 & & & & & 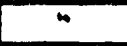 & $\%$ & 5.3 \\
\hline MB.SCR.1.5 & & 1210 & 6 & $350^{\circ}$ & & & & & $\because$ & $\pi$ & 5.0 \\
\hline MB.SCB.1.6 & & 1235 & 7 & $360^{\circ}$ & & & & & " & $\pi$ & 3.4 \\
\hline$M=369.1 .6 A$ & & 1236 & 8 & $360^{\circ}$ & & & & & $" 1$ & $\pi$ & 5.0 \\
\hline & & & & & & & & & & & \\
\hline & & & & & & & & & & & \\
\hline & & & & & & & & & & & \\
\hline & & & & & & & & & & & \\
\hline & & & & & & & & & & & \\
\hline & & & & & & & & & & & \\
\hline & & & & & & & & & & & \\
\hline & & & & & & & & & & & \\
\hline & & & & & & & & & & & \\
\hline & & & & & & & & & & & \\
\hline & & & & & & & & & & & \\
\hline & & & & & & & & & & & \\
\hline & & & & & & & & & & & \\
\hline & & & & & & & & & & & \\
\hline & & & & & & & & & & & \\
\hline & & & & & & & & & & & \\
\hline & & & & & & & & & & & \\
\hline & & & & & & & & & & & \\
\hline & & & & & & & & & & & \\
\hline & & & & & & & & & & & \\
\hline & & & & & & & & & & & \\
\hline & & & & & & & & & & & \\
\hline & & & & & & & & & & & \\
\hline & & & & & & & & & & & \\
\hline & & & & & & & & & & & \\
\hline & & & & & & & & & & & \\
\hline & & & & & & & & & & & \\
\hline & & & & & & & & & & & \\
\hline & & & & & & & & & & & \\
\hline & & & & & & & & & & & \\
\hline & & & & & & & & & & & \\
\hline & & & & & & & & & & & \\
\hline & & & & & & & & & & & \\
\hline & & & & & & & & & & & \\
\hline & & & & & & & & & & & \\
\hline
\end{tabular}


MICROSEEPS LTO.

$$
\begin{aligned}
& \text { CLIENT: SAS/ John Hazbow } \\
& \text { LoCArton: } \frac{M \text { Aveda }}{A / M \text { Crathereovs Aquifen }} \\
& \text { PAGE: } \\
& \text { of: }
\end{aligned}
$$

\begin{tabular}{|c|c|c|c|c|c|c|c|c|c|}
\hline \multirow{2}{*}{ 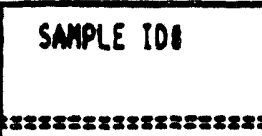 } & \multirow[t]{2}{*}{ OATE } & \multirow[t]{2}{*}{ IIME } & \multirow{2}{*}{ SEO.1 } & \multirow{2}{*}{$\begin{array}{l}\text { SAMPLE } \\
\text { OEPTH }\end{array}$} & \multicolumn{3}{|c|}{ SMMPLE TYPE } & \multirow{2}{*}{$\begin{array}{l}\text { SAPPLE } \\
\text { SIZE }\end{array}$} & \multirow{2}{*}{ COMHENTS } \\
\hline & & & & & 6 & s & 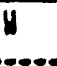 & & \\
\hline$M B-s c r e 2.5 \theta 1$ & 7.8 .9 .93 & 0000 & 1 & 0 & & $\alpha$ & & $40 \mathrm{~mL}$ & $5 \mathrm{ml}$ \\
\hline MB.869.2-1 & & 1359 & 2 & $300^{\circ}$ & & & & & 5.0 \\
\hline 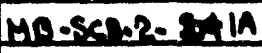 & & 1400 & 3 & 200 & & & & & 5.0 \\
\hline$A B .3 C B .7 \cdot 2$ & & 1410 & 4 & $210^{\circ}$ & & & & 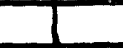 & 5.2 \\
\hline MP.SCB.2.3.3 & \pm & 1433 & 5 & $220^{\circ}$ & & 5 & & 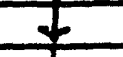 & 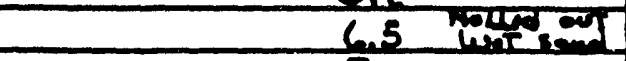 \\
\hline $48.5 c 8.2 .4$ & .299 & 13.5 & 6 & $230^{\circ}$ & & 7 & & T & 5.0 \\
\hline$M B S C Q .2 .5$ & & 1310 & 7 & 240 & & L & & & 5.0 \\
\hline MBscig.2.6 & & 1330 & 8 & $250^{\circ}$ & & E & & & 5.0 \\
\hline $\operatorname{Mr} .5 \mathrm{SCB} \cdot 2 \cdot 7$ & & 1410 & 9 & $260^{\circ}$ & & I & & & 5.0 \\
\hline MBsect 2.8 & & 1450 & 10 & $270^{\prime}$ & & I & & 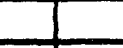 & 5.0 \\
\hline MO.SG8.2.99 & & 1515 & II & $280^{\circ}$ & & I & & & 5.0 \\
\hline$M B . \operatorname{seg} \cdot 2 \cdot 10$ & & 1530 & 12 & $290^{\circ}$ & & & & & 50 \\
\hline$M B-S C B \cdot 2 \cdot 11$ & & 1550 & 13 & $300^{\circ}$ & & I & - & 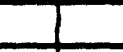 & 5.0 \\
\hline 18-5ch-2-111 & & 1551 & 16 & $300^{\circ}$ & & & & & Fabl wet \\
\hline$M 9.588 .2 .58 .2$ & 3.3 .93 & 700 & 15 & .0. & & & & & 5,01120 anles \\
\hline $2 S C B-2 \cdot 12$ & & 920 & 16 & $310^{\circ}$ & & $t$ & & & $5.0 \mathrm{sic}$ \\
\hline $48.5 C 0.2 .13$ & & 935 & 17 & $320^{\circ}$ & & T & & 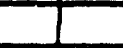 & 5.1 \\
\hline Mnsess.2.14 & & 1045 & 18 & $330^{\circ}$ & & & & & 32 \\
\hline n. $\operatorname{sen} .2 .15$ & & 1100 & 19 & $340^{\circ}$ & & $L$ & & & 5.0 \\
\hline MBSEs:2.142 & & 1125 & 20 & $350^{\circ}$ & & I & & & 5.0 \\
\hline Mpeces.2.17 & 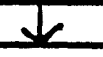 & 1150 & दा & 360 & & \pm & & $\sqrt{k}$ & 5.1 \\
\hline & & & & & & & & & \\
\hline & & & & & & & & & \\
\hline & & & & & & & & & \\
\hline & & & & & & & & & \\
\hline & & & & & & & & & \\
\hline & & & & & & & & & \\
\hline & & & & & & & & & \\
\hline & & & & & & & & & \\
\hline & & & & & & & & & \\
\hline & & & & & & & & & \\
\hline & & & & & & & & & \\
\hline & & & & & & & & & \\
\hline & & & & & & & & & \\
\hline & & & & & & & & & \\
\hline & & & & & & & & & \\
\hline & & & & & & & & & \\
\hline & & & & & & & & & \\
\hline & & & & & & & & & \\
\hline & & & & & & - & & & \\
\hline & & & & & & & & & \\
\hline
\end{tabular}

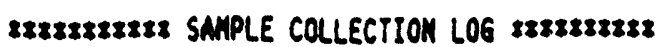

SAPPLER MANE(S): KaR 
MICROSEEPS LTD.
CLIENT: SRS / Jahn Haz Low

LOCATION: M. SREA

PROJECT(8): Soib Coxe Boning

PAGE:

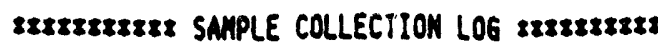

SAMPLER MAME(S): Ka\&

\begin{tabular}{|c|c|c|c|c|c|c|c|c|c|}
\hline SAMPLE IOS & DATE & TIME & SEO.1 & SAMPLE & SAMPI & LET & & SAMPLE & COMMENTS \\
\hline 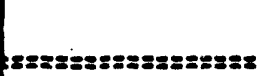 & & & & OEPTH & \begin{tabular}{r|}
6 \\
$323 x 34:$
\end{tabular} & 5 & $\begin{array}{l}4 \\
2228:\end{array}$ & $\begin{array}{c}\text { SIIEE } \\
222232535 x\end{array}$ & Alst urabs inchude $5 \mathrm{mb}$ HeO \\
\hline MO.SC8.4.58.1 & $0.5 \cdot 93$ & 730 & 1 & .0 & & $\checkmark$ & & Sonk & $5.0 \mathrm{H}^{20} \mathrm{cmbr}$ \\
\hline MB.sen-4-1 & & 1234 & 2 & $200^{\circ}$ & & $!$ & & & soil \\
\hline MB.seg. $1 A$ & & 1235 & 3 & $200^{\circ}$ & & - & & & $5.3 \mathrm{Seit}$ \\
\hline MR.sepr.4.2 & & 1250 & 4 & $210^{\circ}$ & & 1 & & & 3.0 Snil \\
\hline$A B \cdot \operatorname{SeA} B .4 .3$ & & 1400 & 5 & $220^{\circ}$ & & $T$ & & & 5.0 \\
\hline He.ses.4.4 & & 1415 & 6 & $230^{\circ}$ & & 1 & & & 5.1 \\
\hline MB.5CQ.4.5 & & 1425 & 7 & $240^{\circ}$ & & $!$ & & & 5.0 \\
\hline MB.5en.4.6e & & 1445 & 8 & $250^{\circ}$ & & $I$ & & & 5.0 \\
\hline Heses.4.7 & & 1505 & 9 & $260^{\circ}$ & & & & & 5.0 \\
\hline$M B . S C 8.4 .8$ & & 1520 & 10 & $270^{\circ}$ & & & & & 5.3 \\
\hline Mescesa.9 & & 1535 & II & $280^{\circ}$ & & & & & 5.2 \\
\hline$M P .5 S R \cdot 5 \cdot 10$ & $v$ & 1550 & 12 & $290^{\circ}$ & & I & & & 5.1 \\
\hline MB.sca.tell & $8 \cdot 4.99$ & 915 & 13 & $300^{\circ}$ & & & & & 5.1 \\
\hline MPSSB A.11A & & 916 & 14 & $306^{\circ}$ & & & & & S.1 \\
\hline HB.SCB.4.5B-2 & & 917 & 15 & .0 & & & & & 5.01120 \\
\hline $18.5 e 8.4 \cdot 12$ & & 935 & 16 & $310^{\circ}$ & & & & & 5.0 \\
\hline $14 . \operatorname{scs} 4.13$ & & 950 & 17 & $320^{\circ}$ & & & & & 5.0 \\
\hline MP.ScP.4.M & & 1015 & 18. & $330^{\circ}$ & & & & & 3.0 \\
\hline MBses $4 \cdot 15$ & & 1020 & 19 & $340^{\circ}$ & & & & & $6.510001)$ \\
\hline MPossed-1he & & 1100 & 20 & $350^{\circ}$ & & & & & 5.0 \\
\hline Mescas.17 & \pm & 1130 & 21 & $360^{\circ}$ & & 12 & & \pm & 30 \\
\hline m.ss..4.18 & & & & & & & & & \\
\hline & & & & & & & & & \\
\hline & & & & & & & & & \\
\hline & & & & & & & & & \\
\hline & & & & & & & & & \\
\hline & & & & & & & & & \\
\hline & & & & & & & & & \\
\hline & & & & & & & & & \\
\hline & & & & & & & & & \\
\hline & & & & & & & & & \\
\hline & & & & & & & & & \\
\hline & & & & & & & & & \\
\hline & & & & & & & & & \\
\hline & & & & & & & & & \\
\hline & & & & & & & & & \\
\hline & & & & & & & & & \\
\hline & & & & & & & & & \\
\hline & & & & & & & & & \\
\hline & & & & & & & & & \\
\hline & & & & & & & & & \\
\hline & & & & & & & & & \\
\hline
\end{tabular}

USE A MEH SHEET WHEN RESTARTING SEOUENCE I 
Attachment C - Headspace (SRTC) Analysis Method Looney et al. (1993) 


\title{
EVALUATION OF HEADSPACE METHOD FOR VOLATILE CONSTITUENTS IN SOILS AND SEDIMENTS
}

\author{
B. B. Looney, C. A. Eddy, and W. R. Sims \\ Westinghouse Savannah River Company \\ Aiken, SC 29808
}

\begin{abstract}
Summary
Detection and delineation of volatile organic contaminants (VOCs) in sediments and soils underlying a hazardous waste site is often a complex problem. The number and quality of analyses used in characterization studies can be compromised by the difficulties and costs associated with quantitative analysis of volatile analytes. A headspace analysis method was developed to facilitate the accurate and rapid delineation of the vertical and horizontal distribution of VOCs in the subsurface, and to reduce the sample handling, laboratory preparation, and analytical complexity associated with most existent sampling and analysis schemes. The headspace method consists of the following four steps:
\end{abstract}

- Subsample the core immediately after retrieval using a small tube/plunger system. - Place the subsample into a $22.5 \mathrm{~mL}$ headspace vial.

- Add $5 \mathrm{ml}$ of suspending solution and cap with a teflon lined septum.

- Analyze an aliquot of the headspace using a gas chromatograph.

This method was developed and modified as a result of multiple site investigations and has been applied to over 2000 samples from both saturated and unsaturated sediments. Data collected during these studies indicated that distilled water suspending solution is similar to an ionic $\left(\mathrm{Na}_{2} \mathrm{SO}_{4} / \mathrm{H}_{2} \mathrm{PO}_{4}\right)$ solution and that sonication of the samples does not enhance the recovery of VOCs. Sealed samples exhibited stabi'; concentrations for more than 20 days. To further evaluate the headspace method, 92 pairs of samples were analyzed to allow direct comparison of the headspace method to a modified EPA solvent extraction method typically applied to environmental samples. Despite the precautions incorporated into the solvent extraction method, the results indicated that sample transfers in the field and laboratory resulted in substantial volatilization of VOCs. The headspace method minimized these losses and generated results rapidly, facilitating informed decision making during site characterization.

\section{Background}

Barcelona (1989) suggests that sample collection and handling activities can contribute to systematic errors in environmental data. These errors are often relatively large compared to the random and systematic errors typically associated with the instrumental analysis. Perhaps the most difficult sample collection and handling error to delineate and control is negative bias (measured value less than true value). In the case of VOCs, this error is principally caused by volatilization of the analyte during sample collection, storage, and handling. In samples from the saturated zone, displacement of pore water by drilling fluids 
or drainage of the core can contribute to negative bias. Recent research (Seigrist and Jenssen, 1990; Urban et al., 1989) indicates that the typical methods (containerization of disturbed samples, followed by refrigerated storage and solvent extraction) may lead to substantial volatilization loss; the investigators recommended controlled research and development of alternate procedures.

Analytical methods for VOCs in water samples can logically be grouped into the following three categories:

- solvent extraction

- static headspace methods

- dynamic headspace methods (purge and trap)

Each of these categories relies on a partitioning of the contaminant from the water into an alternate phase prior to instrumental analysis. In the case of solvent extraction, the alternate phase is typically a liquid organic solvent, while in the headspace categories, the alternate phase is a volume of gas. The success of an analytical method depends on the relative affinity of the VOC for the alternate phase, the compatibility of the extracting phase with the analytical instrumentation, and the ability to reproducibly contact the phases and handle the extract. For solvent extraction and static headspace methods, an aliquot of the extract is generally introduced into a gas chromatograph (GC) equipped with an appropriate detector or mass spectrometer. In purge and trap analysis, gas is bubbled through the sample at a constant rate for a specified time. Contaminant vapors are collected on an adsorbent trap; following the purge, the contaminants are thermally desorbed into the GC.

Existing analysis methods for soil and sediments are predominantly in the solvent extraction class; however, both static headspace and purge and trap methods are documented in the literature (McNally and Grob, 1985). Successful application of the gas phase extraction methods to soils and sediments relies on effective and reproducible partitioning from the solid to the gas phase for dry samples, and from the solid and liquid phases to the gas phase for wet samples.

The relative simplicity and minimal sample handling suggest that the static headspace method may be a relatively attractive technique for analysis of volatile constituents in soil and sediment samples. Static headspace methods are most applicable to samples with mineral or low organic matrices. Additionally, these methods require relatively constant conditions for reliable proportionality between original sample concentration and the mass of each VOC introduced into the GC. In particular, the properties of any suspending solution (e.g., ionic strength, $\mathrm{pH}$, etc.) and the physical conditions in the vial (e.g., temperature, pressure, phase volumes, etc.) should be selected to minimize sorption and maximize the conditional Henry's Law partitioning from the solution to the gas phase.

Reports in the literature document successful application of static headspace methods to water, wastewater, industrial effluents, soil, sediments, and sewage (McNally and Grob, 1985). In cases where reproducible results are documented, headspace methods are often preferred because they are simpler and faster, and therefore less expensive than either solvent extraction or purge and trap methods. Since sample handling can be minimized and analyses are generated rapidly, results of this screening approach can be incorporated during the site characterization process. 


\section{Methods and Study Design}

The sediments for the headspace sampling and analysis studies were collected from borings at sites in the vicinity of the metallurgical manufacturing facility in M-Area at the Savannah River Site (SRS). Solvents -- trichloroethylene (TCE) and tetrachloroethylene (PCE) -were used in this facility during the late 1950s to the early 1980s to degrease the fuel and target tubes prior to use in other facilities at SRS. Concentrations of VOCs in the partially saturated and saturated sediments vary vertically and horizontally beneath the site. Proper site characterization and long term remediation system design requires adequate delineation of this variation.

The boring locations in M Area were selected based on results from a shallow soil gas survey, combined with process records, groundwater data and past core data. The overall method development study consisted of two parts. First, following an initial period of method development, a series of samples was collected and analyzed to optimize the conditions for sampling and analysis. Second, a series of paired samples was collected to compare the headspace method to a solvent extraction method typically used for soilsediment analysis. A brief discussion of the headspace method is provided below, followed by specific details associated with each phase of the study.

\section{Drilling and Coring}

Continuous borings were drilled to an approximate depth of 200 feet at each location using two drilling methods. Within the vadose zone (130 - 140 foot depth), samples were collected using 4.25-inch inside diameter hollow-stem augers and a standard 2-inch inside diameter, split-spoon sampler. Below the water table, the boreholes were stabilized using a (bentonite-based) mud rotary system, and continuous samples were collected ahead of the borehole using a CP wireline system and Christensen Core Barrel. All subsamples for VOC analysis were collected as quickly as possible after the core was retrieved.

\section{Headspace Sampling-Analysis Procedure}

The headspace sediment subsample (3-5 grams) was collected immediately from the open split-spoon using an open-ended plastic disposable syringe and extruded into a $22.5 \mathrm{~mL}$ borosilicate vial. Using as pipet, $5 \mathrm{~mL}$ of suspending solution were added to the subsample and the vial was sealed by crimping an aluminum cap around a teflon-lined butyl rubber septum. The sample vial was labeled and placed in an ice chest cooled to approximately $4^{\circ}$ $\mathrm{C}$ for later analysis at an onsite laboratory. The subsample corer (syringe) was decontaminated between sampling events by brushing and rinsing with isopropanol followed by a distilled water wash.

The onsite laboratory consisted of a headspace analyzer connected to a Hewlett Packard (HP) 5890A Gas Chromatograph (GC). Details of the headspace analysis procedure used are given below. Prior to field sampling, we determined the average weight of a sealed headspace vial containing five milliliters of suspending solution. Upon receipt of the headspace sample vials from the field, the capped vials containing the sediment samples were weighed. The amount of sediment in each vial was determined by subtracting the average weight from the sample weight. Each vial with the sediment subsample was analyzed using the HP GC equipped with an electron capture detector, an HP 19395 headspace sarnpler, an HP 3392 networking integrator, and a $60 \mathrm{~m}$ widebore capillary 
column coated with a nonpolar silicone phase. The flow and oven temperature conditions recommended by the manufacturers were used. The instrument was calibrated using vials containing known quantities of VOCs, suspending solution, and (in some cases) clean representative sediments. The conditions in the vials (headspace volume, suspending solution volume, and temperature) were standardized as much as possible to maintain the proportionality between the sample concentration and VOC mass in the headspace aliquot. The heated $\left(70^{\circ} \mathrm{C}\right)$ bath in the headspace sampler maximizes the transfer into the vapor phase. The data for each peak was entered into a spreadsheet and the concentration of contaminant in the original sample was estimated using the response factors from the calibration. All values were reported in units of micrograms of VOC per gram of bulk sediment $(\mu \mathrm{g} / \mathrm{g})$. Approximately 30 to 50 samples were analyzed each day.

\section{Headspace Method Optimization Study}

The purpose of this phase of the project was to identify the most effective operating conditions for the three phase static headspace procedure. Specifically, the nature of the suspending solution and the new for physical agitation were analyzed. In each case, a reference condition was identified and the relationship between the reference and alternate conditions were evaluated by comparing the relative recoveries for a large number of sample pairs. Because of documented superiority of adding salt when analyzing water samples (Gottauf, 1966), a Na2 $\mathrm{SO}_{4} / \mathrm{H}_{2} \mathrm{PO}_{4}$ buffer solution was selected for the reference suspending solution ( $200 \mathrm{~mL}$ distilled water, $10 \mathrm{~g}$ sodium sulfate, and $0.3 \mathrm{~mL}$ concentrated phosphoric acid) and was compared with the distilled water. The reference physical agitation method was sonication, which was compared with the alternate method of no agitation (i.e., the vials were placed directly into heated headspace bath). Additionally, a time-series study was performed to determine the stability of the sediment samples sealed in headspace vials.

\section{Comparison of Headspace Method to Solvent Extraction}

The two separate laboratories utilized in the study to analyze the sediment subsamples were an onsite laboratory operated by Savannah River Laboratory personnel and a close support laboratory (CSL) operated by independent subcontract personnel. The onsite laboratory analyzed the headspace sediment subsamples and the CSL analyzed the sediment subsamples collected by the independent subcontractor. Both laboratories used standard chain-of-custody procedures and collected quality assurance/quality control (QAVC) sediment subsamples to comply with the SRS QA requirements. These requirements included the analysis of duplicate samples, matrix spikes, and trip blanks. All analyses were performed within the required holding time. The method selected by the subcontractor for the CSL was typical of those applied at waste sites in the United States (EPA method 3550). The method generally consisted of containerization of disturbed samples followed by refrigerated storage, sample transfer, and solvent extraction.

During this study, water was used for the headspace suspending solution and the headspace samples were not sonicated. The results from the headspace analysis and CLS were used to determine screen intervals for vapor extraction wells installed as part of a vadose zone remediation program. 


\section{Results and Discussion}

The data suggest that a headspace analysis approach provides rapid and reproducible analytical results for analysis of VOCs in many common soils and sediments. The parameter optimization phase of the study indicated that a distilled water suspending solution is similar to a $\mathrm{Na}_{2} \mathrm{SO}_{4} / \mathrm{H}_{2} \mathrm{PO}_{4}$ suspending solution and that sonication of the sample does not improve the transfer of contaminant into the headspace from the solid/liquid phases. The time-series data suggested that samples are relatively stable following collection; the replicate vials generated similar concentrations for the entire time-series period of 20 days. Elimination of the buffer solution and sonication step, based on the parameter optimization phase, yields a sampling/analytical scheme that is rapid and simple to implement.

In a second phase of the study, the headspace method was directly compared to a modified EPA solvent extraction method. Despite the precautions incorporated into the solvent extraction method, the analytical results indicated that sample transfers in the field and laboratory resulted in significant volatilization of VOCs from the sediment samples prior to analysis. The headspace method appears to provide more representative data on the samples. The headspace analysis method generally resulted in a higher value for the measured concentration of both TCE and PCE. The two primary exceptions to this general trend are samples with very high concentrations of contaminants and samples where both methods were below detection limits. For example, in the samples from one of the cores, there are five examples where the two methods are the same for TCE. All of these examples are found where the analytical results are below detection limits for both methods. Similarly, in this core, the results from the solvent extraction method are greater in only 4 of 33 examples. All 4 examples result from overloading of the GC during the headspace analysis (the samples can not be diluted). These same trends may be observed in all of the other cores. In the comparison study, the headspace method indicated the presence of contamination in each of the silty, clayey, and poorly graded layers throughout the vadose zone. The solvent extraction method generated below detection results for most of these zones. Additionally, the headspace method indicated low (but measurable) concentrations in the well-graded sands, while the solvent extraction method indicates below detection results in almost all of these layers.

The paired data were ranked and ordered for statistical analysis. In this form, a Wilcoxon Signed Rank Test was applied to determine if the two methods yielded statistically similar results. This hypothesis was rejected at greater than the $99 \%$ confidence level, signifying that the two populations are different. Thus, the statistical test indicated that there is greater than a $99 \%$ probability that the two methods are statistically different (i.e., the headspace method generates higher values).

As discussed above, one limitation of the headspace method is that the sample can not be diluted; thus, very high concentrations are truncated by an upper limit of detection. In most cases, this truncation may not be of practical significance because it occurs at relatively high concentrations (e.g., 100,000 ng/g). This truncation can be essentially eliminated by splitting the column effluent to a flame ionization detector (FID) in parallel with a halogen specific detector. In this configuration, the less sensitive FID extends the range of the analysis by several orders of magnitude. 


\section{Conclusions}

The results indicate that the headspace method minimized loss of volatiles associated with sample handling and provided large amounts of closely spaced data. From an analytical standpoint, at sites with low sediment organic carbon and relatively volatile constituents, there are several advantages of the headspace method over solvent extraction methods. Some of these advantages include the following:

- reduced sample handling effort and time in the field

- no solvent extraction required (the Henry's Law mass transfer in the headspace vial requires no operator effort)

- elimination of multiple sample transfers and minimization of the opportunities for volacilization of analyte

The headspace sediment sample is sealed in its final form ready for analysis within a few seconds of collection and is never directly handled again during weighings or transfers. Once in the laboratory, approximately 50 samples can be analyzed in a normal working day on a single instrument. Headspace analysis is cost effective; we have calculated the fully loaded costs of the analysis to be $\$ 50$ - $\$ 100$ per sample. In addition, the headspace results can be generated rapidly and transferred to the field so that informed decisions can be made during site characterization.

The information contained in this article was developed during the course of work under Contract No. DE-AC09-89SR18035 with the U. S. Department of Energy. By acceptance of this paper, the publisher and/or recipient acknowledges the U.S. Governments right to retain a nonexclusive, royalty-free license in and to any copyright covering this paper along with the right to reproduce, and to authorize others to reproduce all or part of the copyrighted paper. 


\section{REFERENCES}

Barcelona, M. J., 1989. Report in Principles of Environmental Sampling. L. H. Kieth editor, American Chemical Society, Washington DC, 3-23.

Gottauf, M., 1966. Verbesserte quantitative gaschromatographische Spurenanalyse flüchtiger organischer verbindungen in wasser. Fresenius' Zeitschrift für Analytische Chemie, 218, 175-184.

Hachenberg, H and A. P Schmidt, 1977. Gas Chromatograsphic Headspace Analysis, Heyden Publishers, Philidelphia PA.

Ioffe, B. V., and A. G. Vitenberg, 1984. Headspace Analysis and Related Methods in Gas Cromatography. John Wiley and Sons, New York.

Kerfoot, H. B., 1991. Groundwater, 29, pp 678-684.

Kolb, B., 1980. Applied Headspace Gas Chromatography. Heyden Publishers, Philidelphia PA.

McNally, M. E. and R. L. Grob, 1985. Current applications of static and dynamic headspace analysis: part 1: environmental applications. American Laboratory, January 1985, 20-33.

Siegrist, R. L. and P. D. Jenssen, 1990. Evaluation of sampling method effects on volatile organic compound measurement in contaminated soils. Environmental Science and Technology, 24:9, 1387-1392.

Urban, M. J., J. S. Smith, E. K. Schultz and'R. K. Dickson, 1989. Report in Fifth Annual Waste Testing and Quality Assurance Symposium; U. S. Environmental Protection Agency, Washington DC, II87-II101.

EPA, 1986. Test Methods for Evaluating Solid Waste, Physical/Chemical Methods. SW. 846. U. S. Environmental Protection Agency, Office of Solid Waste and Emergency Response, Washington, D. C. 


\section{BIOGRAPHICAL SKETCHES}

B. B. Looney received his $\mathrm{PhD}$ in Environmental Engineering from the University of Minnesota in 1983. He is currently employed as a research engineer in the Savannah River Laboratory and is an adjunct professor in the Environmental Systems Engineering Program at Clemson University. His interests and responsibilities include, developing and testing new methods for environmental characterization and remediation, risk assessment, and modeling. Westinghouse Savannah River Company, 773-42A, Aiken SC 29808.

C. A. Eddy is completing her PhD in geology at University of Califomia, Davis. She is currently employed as a research geologist at the Savannah River Laboratory. Her interests include geology and analytical geochemistry. Westinghouse Savannah River Company, 773-42A, Aiken SC 29808.

W. R. Sims received his MS degree in Geology from the University of Akron in 1987. He is currently a hydrogeologist in the Environmental Restoration Department at the Savannah River Site. His responsibilities and interests include RCRA/CERCLA waste site assessments, vadose zone and groundwater characterization and remediation, and regulatory support for groundwater corrective actions. Westinghouse Savannah River Company, Environmental , Aiken SC 29808 
Attachment D - Purge and Trap (MicroSeeps Ltd) Analysis Method EPA Method 5035 (draft) 
METHOD 5035

MODIFIED PURGE-AND-TRAP AND EXTRACTION FOR VOLATILE ORGANICS IN SOIL AND WASTE SAMPLES

\subsection{SCOPE AND APPLICATION}

1.1 This method describes a modified purge-and-trap process for the analysis of low concentrations of volatile organic compounds (VOCS) in soils/sediments and solid waste. Guidance is also provind for sample preparation of soils, solid waste and non-aqueous $1 /$ with high concentrations of volatile organics. The gas chromatographic eterminative steps are found i.1 Methods 8010,8015, 8020,8021 and 8030 (Tat V). The method is also applicable to GC/MS Methods 8240, 8260, and 8266 .

1.2 The low soil method differs from the loy $1 /$ seditim nethod in the original Method 5030 because the hermetic seal of saple vial ver broken from time of sampling to time of analysis. Sinf the sample is nev rposed to the atmosphere after sampling, the loss of $\mathrm{NOCs}$ is negligible. fore, concentration data obtained using Method 50 would be ypected to be 9 gher and more representative of the soil contami, at ti, sampling, than that obtained using the original low soil metrod sub mpling a portion of sample from the sample vial in the laboratory). The cable concentration range of the low soil method is dependent on the det ative method, matrix, and compound. However, it will generally fall in the to $200 \mathrm{~g} / \mathrm{kg}$ range. The estimated quantitation limit nae for high concent. an analysis of soil and waste samples will be in the
dependent on interferences.

\subsection{Method 5035 can be use for}

organic compounds that have boiling points below $200^{\circ} \mathrm{C}$ and at soluble of slightly soluble in water. Volatile, water-soluble compounds $c$ be incluoded in this analytical technique; however, quantit? mits (by GC, GC/MS) are approximately ten times higher because of poo purgith ficiency. method is also limited to compounds that elute as shy, peaks in a GC colum acked with graphitized carbon lightly coated wity rbowax a coated cap hary column. Such compounds include low molecular we, ha acetates, acryl. etimentides.

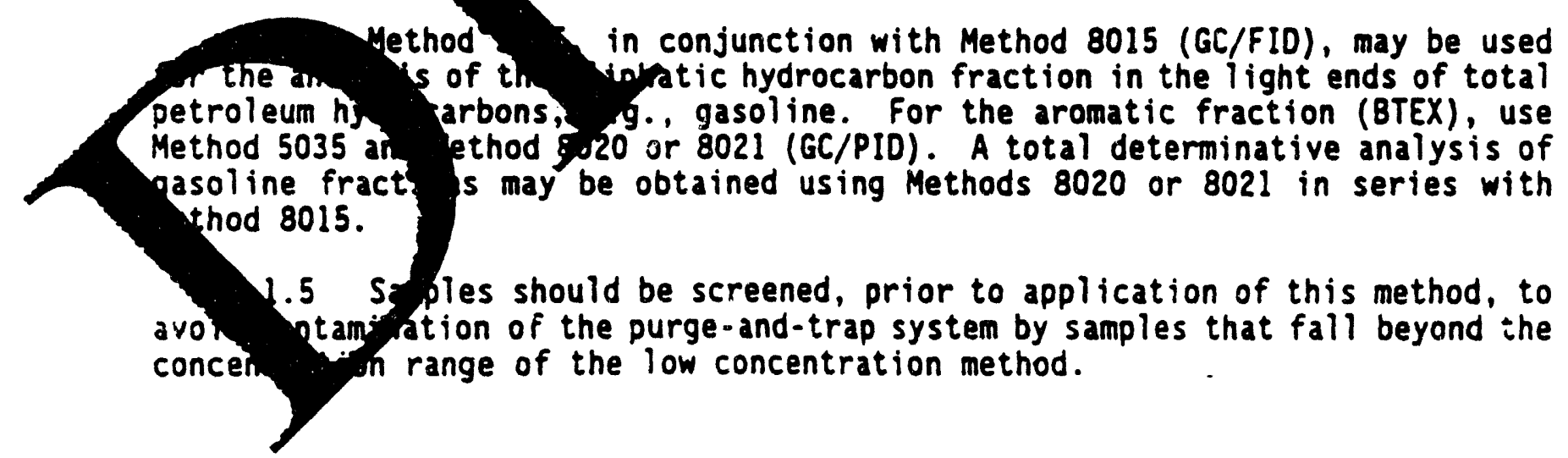




\subsection{SUMMARY OF METHOD}

2.1 Low Concentration Method: Volatile organic compounds (VOCs) are determined from a $5 \mathrm{~g}$ soil sample by placing the sample, at time of collection, into a specially designed, fritted, $40-\mathrm{mL}$ vial. A stirring bar is added and, if desired, preservative may be added as well. The vial is then sealed and shipped to a laboratory or appropriate analysis site. The entire vial is then placed, unopened, into the instrument carousel. Immediately before analysis, water, surrogate standards and internal standards are automatically added without breaking the hermetic seal on the sample vial. The slurry is preheated to $4 C^{\circ} \mathrm{C}$, then purged by passing an inert gas through the bottom of vial while mechanical agitation is being provided by the magnetic stiry ing bar. Purged components then travel via a transfer line to a trap. When $p$ ging is complete, the trap is heated and backflushed with helium to desor trapped sample components into a gas chromatographic (GC) column nteri. to a mass spectrometer (MS) or a specific detector, depending on fe deter. tive method selected.

2.2 High Concentration Method: If the mple introduction tec in Section 2.1 is not applicable, a portion of sample dispersed in water miscible solvent to dissolve the volatile g. ic const ents. An al lavot of the solution is combined with water in a specr. desined purging chamber. It is then analyzed by purge-and-trap GC following cer purge-and-trap method (Method 5030).

\subsection{INTERFERENCES}

3.1 Impurities in the purg gas organit compounds out-gassing from the plumbing ahead of the tra accour fiajority of contamination problems. The analytical system mus be dt nstrated to be free from contamination under the conditions of analysi by running laboratory reagent blanks. The use -polytetrafl, roethylene (non-PTFE) plastic coating, non-PTFE thread or flow cor rollers with rubber components in the purging device ast be a ded, since s h/materials out-gas organic compounds which will b encentra of in the tra during the purge operation. These compounds wrin. ult in Interferences or false positives in the determinative step.

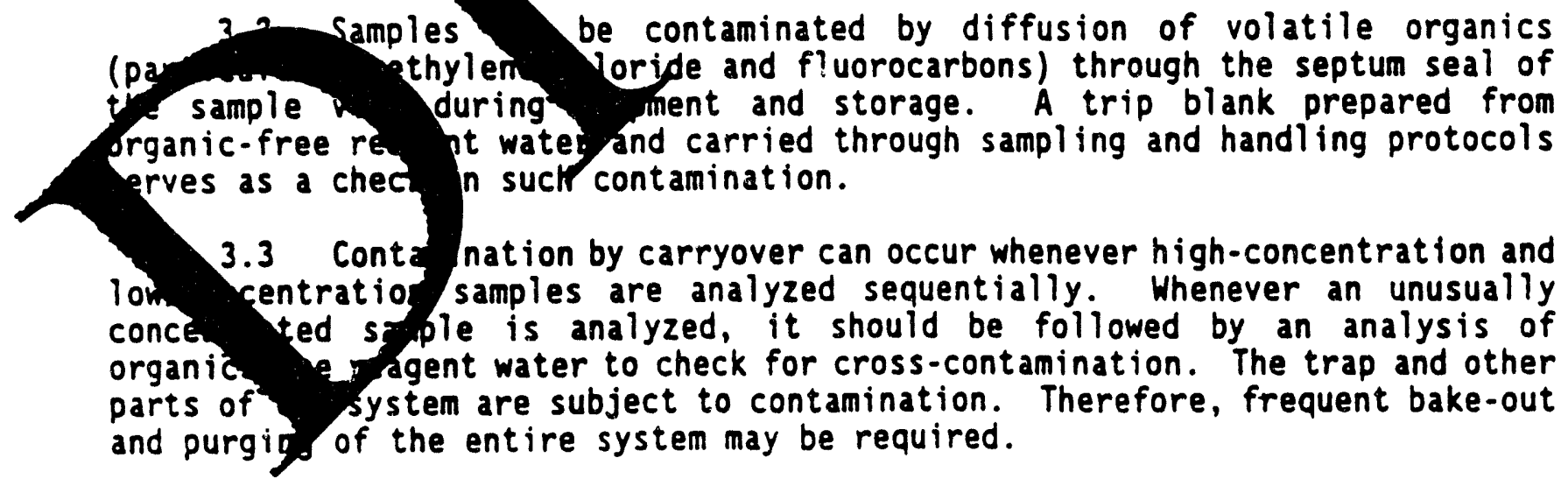


3.4 The laboratory where volatile analysis is performed should be completely free of solvents. Special precautions must be taken to determine methylene chloride. The analytical and sample storage area should be isolated from all atmospheric sources of methylene chloride, otherwise random background levels will result. Since methylene chloride will permeate through PTFE tubing, all GC carrier gas lines and purge gas plumbing should be constructed of stainless steel or copper tubing. Laboratory workers' clothing previously exposed to methylene chloride fumes during common liquid/liquid extraction procedures can contribute to sample contamination. The presence of other organic solvents in the laboratory where volatile organics are analyzed will also lead to random background levels and the same precautions must be tyen.

\subsection{APPARATUS AND MATERIALS}

4.1 Sample Containers/Purge Device - 40-ml Clear special frit (Figure 1) available from Oynatech Pre. sion Sam. Each vial must be equipped with o TFE-faced Corporation which demonstrate minimal bleed at elevated typeratures. Prior. "se, wash vials and septa with detergent and rinse with ap and distilled water. low the vials and septa to.air dry at room temper ure, plac in a $105^{\circ} \mathrm{C}$ ov r for one hour, then remove and allow to cool in anown free of organics. Be sure the PTFE side of each septum is towar. sa ple.

4.2 Purge-and-Trap System - The systelind for purging and trapping consists of two pieces of equipment linked togethe. form a hybrid system. The first piece of equipment perfor as the automated sa device while the other piece of ant contains th. ap and functions as the desorber. Systems are commercia ly from sey_ral sources that meet all of the following specifications.

NOTE: The equipment used to ay lop this ethod was a Dynatech PTA-30 W/S Autosamnler (Dynatech ecision Sampling Corporation, 8275 West E1

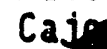

2.1 The

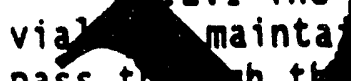

pass th.

capable

ourging der Baton Roug LA 70815).

rging device $A$ be capable of accepting the $40-\mathrm{mL}$ soil ing the vial at $40^{\circ} \mathrm{C}$ while the inert gas is allowed to fencively purging it. The device must also be crourom $\mathrm{mL}$ of organic-free reagent water into the ithout venting the headspace of the vial. It must also be ing the sample during purging. The analytes being purged mus allow. maint. of an avated temperature. After passing through the transfer line, th analyts are then allowed to concentrate on a trap.

4. 2 The trap used to develop this method was 25 centimeters long, had an inside diameter of 0.105 inches and was packed with Carbopa/Carbosieve (Supelco, Inc.). Traps that demonstrate similar udrop obic and retention properties may be used. The trap must strate sufficient adsorption and desorption characteristics to meet t. method MOLs of all the target analytes for a given Project and the QC equirements in Method 8000 and the Determinative Method. The most difficult are the gases and especially dichlorodifluoromethane. Also,

$$
5035-3
$$


demonstrate that the trap is capable of desorbing the late eluting target analytes.

NOTE: Check the response of the brominated compounds when using these alternative charcoal traps (especially Vocarb 4000), as some degradation has been noted relating to the higher desorption temperatures (especially temperatures above 240 . $250^{\circ} \mathrm{C}$ ). 2-Chloroethyl vinyl ether is degraded on Vocarb 4000 but performs adequately when Vocarb 3000 is used. The primary criteria, as stated above, is that all target analytes meet the MOL requirements for a given project.

4.2.2.1 The desorber for the above trap ast be capable of rapidly heating the trap to $245^{\circ} \mathrm{C}$ prior to the $\mathrm{c}$. ning of the flow of desorption gas. Several comercial des otin ourge-and-trap units) are available.

4.2.3 The standard trap used in previoy of purge-and - methods is also acceptable. This trap is $25 \mathrm{~cm}$ lone and has an inside d teryof at least $0.105 \mathrm{in.}$ Starting from the nlet, the trap cont the following amounts of adsorbents: 1/3, 2,6-diph yylene oxide o ymer, $1 / 3$ of silica gel, and $1 / 3$ of cocony rcoal. sis recommented that $1.0 \mathrm{~cm}$ of methyl silicone-coated pack. be iserted at the inlet to extend the life of the trap. If it is necessary to analyze for dichlorodifluoromethane or other fluorocarba. similar volatility, the charcoal can be eliminated and the polymer in sed to fill $2 / 3$ of the trap. If only compounds beling above $35^{\circ} \mathrm{C}$ are analyzed, both the silica gel and charcoal can piminated and the polymer increased to fill the entire trap. Before ining this tras should be conditioned overnight at $180^{\circ} \mathrm{C}$ by backfluping $w$, iners gas flow of at least 20 $\mathrm{mL} / \mathrm{min}$. Vent the trap effluent to th hoou, to the analytical column. Prior to daily use, the trap sho lo we condit, oned for 10 min at $180^{\circ} \mathrm{C}$ with backflushing. The trap may be ented to the analytical column during daily condit however, e column must be run through the temperature progra, fior to anal is of samples.

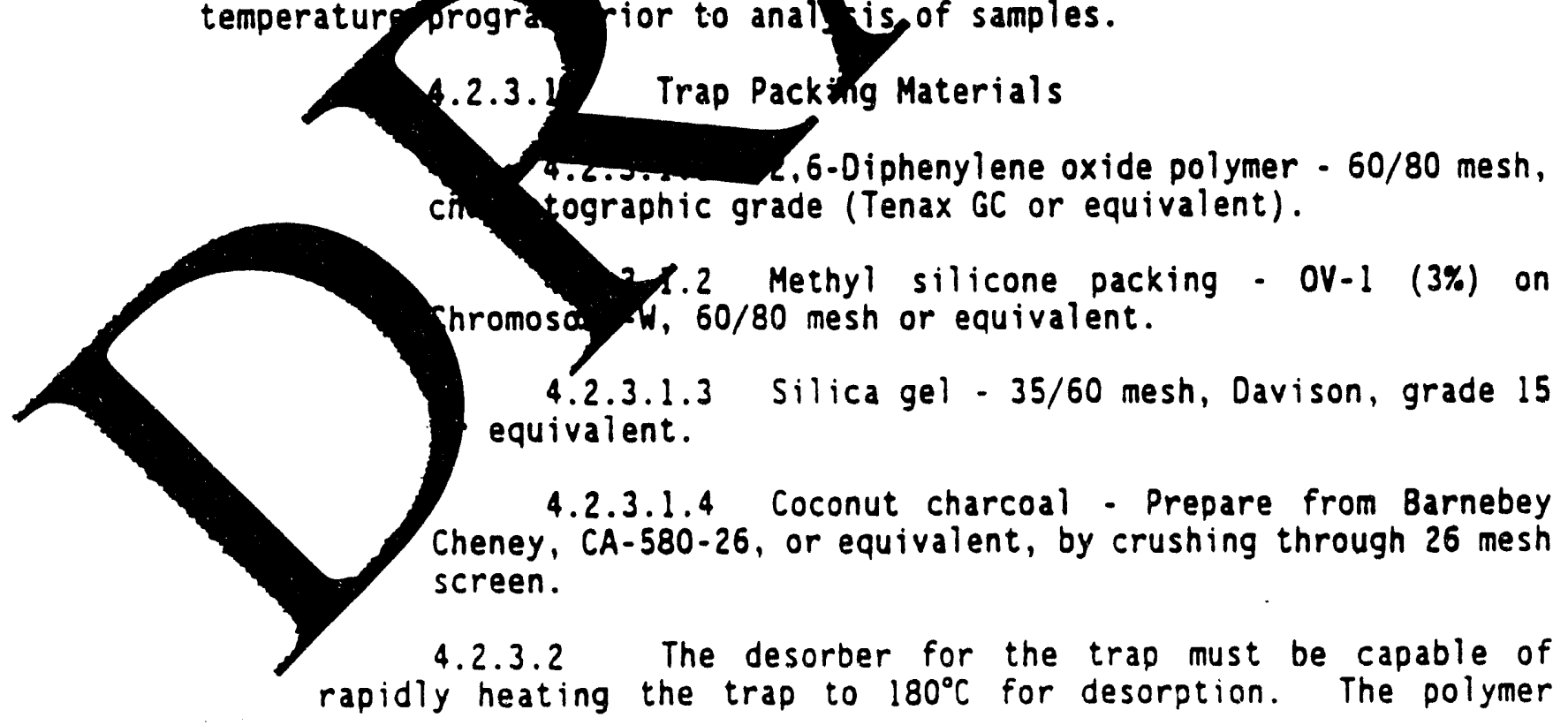


section of the trap should not be heated higher than $180^{\circ} \mathrm{C}$, and the remaining sections should not exceed $220^{\circ} \mathrm{C}$ during the bake-out mode.

4.2.3.3 Prior to initial use, condition the trap overnight at $180^{\circ} \mathrm{C}$ in the purge mode with an inert gas flow of at least 20 $\mathrm{mL} / \mathrm{min}$. Prior to daily use, condition the trap for $10 \mathrm{~min}$ while backflushing at $180^{\circ} \mathrm{C}$ with the GC column at $220^{\circ} \mathrm{C}$.

\subsection{Syringe and Syringe Valves}

4.3.1 Two $25-\mathrm{mL}$ glass hypodermic syringes Luer-Lok (or equivalent) tip (other sizes are acceptable dependis on sample volume used).

4.3.2 Three 2-way syringe valves with Ly end.

4.3.3 One 25- L micro syringe with 2 peh $\times 0.0$ inch ID, 22 bevel needle (Hamilton $\$ 702 N$ or equivale 1 .

4.3.4 Micro syringes - 10,100 C.

4.3.5 Syringes - $0.5,1.0$, dillu, ga chigh with shut-off valve.

\subsection{Miscellaneous}

4.4.1 Glass vials- $60 \mathrm{~mL}$, septum se. to collect samples for screening, dry weight de cination, and high, entration analysis (if
needed).

\subsubsection{Top-loading ba ince}

4.4 .3 Glass scintillat. vials - $20 \mathrm{~mL}$, with screw-caps and Teflon liners or al culture tubes with screw-caps and Teflon liners.

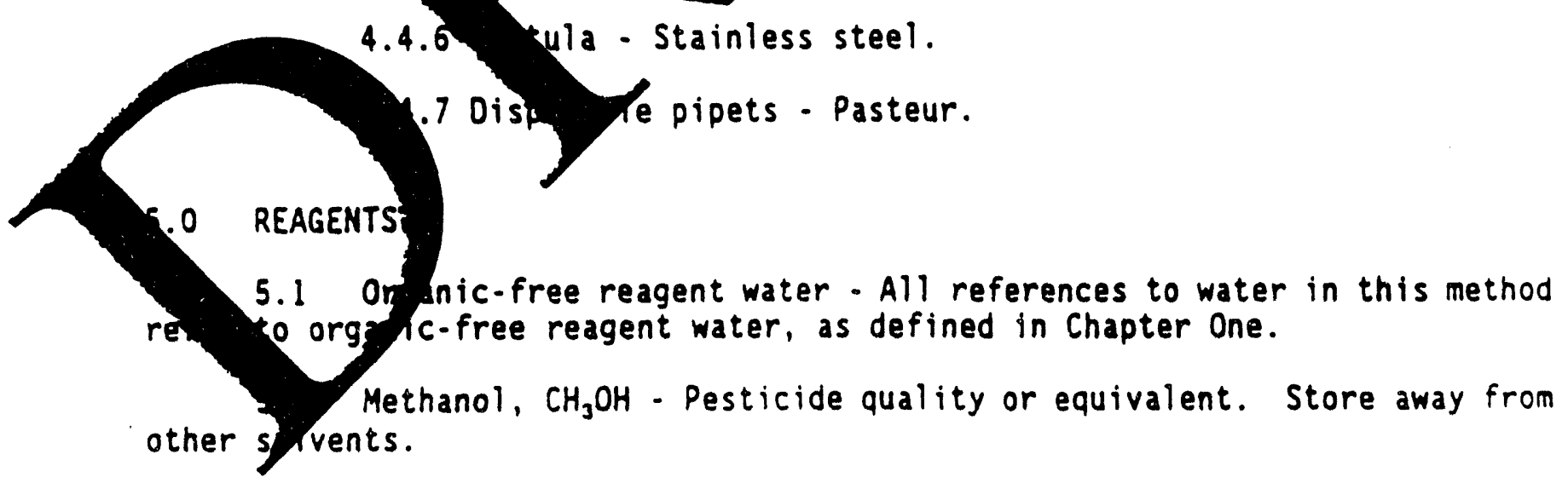
GC autosampler. 
5.3 Polyethylene glycol, $\mathrm{H}\left(\mathrm{OCH}_{2} \mathrm{CH}_{2}\right)_{n} \mathrm{OH}$ - Free of interferences at the detection limit of the target analytes.

5.4 See the determinative method and Method 3500 for guidance on internal and surrogate standards.

6.0 SAMPLE COLLECTION, PRESERVATION, AND HANOLING

\subsection{Sample Collection}

6.1.1 Weigh the assembled soil sample vial containg the stirring bar to $0.1 \mathrm{~g}$. Ship the tared sampling vial to the sag ing site with the seals intact. Open the large chamber containing the ng bar, and add about 5 grams $(2$ - 3 centimeters) of soil on top 9 the sh ing bar (wear gloves whenever handling the tared containers (mmedi $x$ seal and store at $4^{\circ} \mathrm{C}$. (Samples may be weighed in the f d f a means vailable to weigh to $0.1 \mathrm{~g} .1$ Do not interchange seal and tirring bars hother soil vials. It is advisable to collect plicate samples in th. fial tared sample/purge vials in case reanals sis of th sample is requ red.

6.1.2 Collect additional duplrat. iquot of each sample in $60 \mathrm{~mL}$ glass vials (septum sealed) for screening. Weight determination, and high concentration analysis (if needed).

\subsection{Sample Storage}

6.2.1 Store samples at must be free of organic solve t vapu.

6.2.2 All samples should pe nalyzed thin 14 days of collection. Samples not analyzed within s period must be noted and data are

7.0 considered mi ralues.

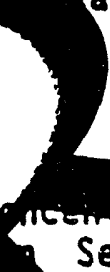

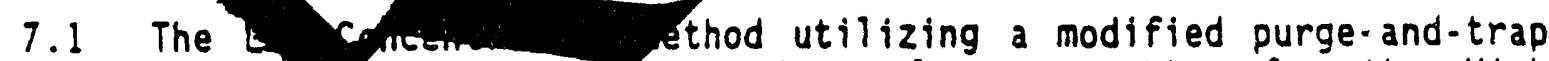
technique is fourt Section 7.2 and sample preparation for the High Conce Hethod found in section 7.3. The gas chromatographic de Miminatim are and in Methods 8010,8015,8020,8021 and 8030 (Table 1. The metho al so ap. table to GC/MS Methods 8240,8260 and 8266 . For the analys is of gasa. $e$, use kethod 8020 or 8021 with GC/PID for BTEX in series with thod 8015 with e GC/FID detector for hydrocarbons.

Y.2 Low $C$ icentration Method for Soil/Sediment and Solid Waste Amenable to $\mathrm{b}$ ipdified arge-and-Trap Method (Approximate concentration range of 0.5 to 200 . $\mathrm{kg}$ - Fe concentration range is dependent upon the determinative method and the Avity of each analyte.)

7.2.1 Initial calibration: Prior to using this introduction technique for any GC or GC/MS method, the system must be calibrated. General calibration procedures are discussed in Method 8000, while the 
determinative methods and Method 3500 provide specific information on calibration and preparation of standards. Normally, external standard calibration is preferred for the GC methods because of possible interference problems with internal standards. If interferences are not a problem, based on historical data, internal standard calibration is acceptable. The GC/MS methods normally -tilize internal standard calibration. The GC/MS methods require instrument tuning prior to proceeding with calibration.

7.2.1.1 Assemble a purge-and-trap device that meets the specification in Section 4.2 and is intep ced to a gas chromatograph or a gas chromatograph/mass sumeter system. Before initial use, a Carbopack/Carbosi trap should be conditioned overnight at $245^{\circ} \mathrm{C}$ by backflue with an inert gas flow of at least $20 \mathrm{~mL} /$ minute. (If oth ing materials are substituted for the Carbooack/Carbosiey foito manufacturers recommendations for conditioning. See ction 4.2. for guidance on conditioning the trap.) Vent the apeffluent a room, not to the analytical column. Prior o dany use, the $h$ shald be conditioned for 10 minutes at 24 with backflushing. Irap may be vented to the analytical column d ing daily cositioning; however, the column must b throur temperature program prior to analysis of samples.

7.2.1.2 To prepare a c. Tation standard, inject an appropriate volume of a primary d ion standard (containing analytes and surraates) to an aliquot a yc-free reagent water in a volumetric f. gas tight syr, or to $10 \mathrm{~mL}$ of this solution in a soi and inject in appropriate amount of internal standards the the rese reagent water. Be sure that the same amount of in rnal aliure added to each standard and sample. The volume of organic, ree reagent water used for calibration must be same volume used for sample analysis (norma2 $10 \mathrm{~mL}$ ). The furrogate and internal standard solutions mus with a syr ge needle long enough to ensure addition br ow the face of the Ar. Prior to purging, heat the sample

91 to $40^{\circ}$ for 1.5 minu $s$ (the analytes in Method 8030 normally ire an ser nurae temperature).

E: The device on the autosampler that introduces the solution containing the internal standards and urragates must be disabled during calibration. Aqueous ndards ire not stable and should be discarded after ne hour unless transferred :0 a sample bottle (or gas tight syringe) with no hea:: :e and sealed immediately.

7.2.1.3 Carry out the purge-and-trap procedure as out lined in ction 7.2 .4 .4 .

7.2.1.4 Calculate response factors (RF) or calibration ractors (CF) for each analyte of incarest using the procedure described in Method 3000. 
7.2.1.5 The average CF (external standards) or RF (internal standards must be calculated for each compound. For GC/MS analysis, a system performance check must be made before this calibration curve is used (see Methods $8240 / 8260 / 8266$ ). If the purge-and-trap procedure is used with Method $8010 / 8021$, evaluate the response for the following four compounds: chloromethane; 1,1-dichloroethane; bromoform; and 1,1,2,2-tetrachloroethane. They are used to check for proper purge flow and to check for degradation caused by contaminated lines or active sites in the system.

7.2.1.5.1 Chloromethane: This compoy is the most likely compound to be lost if the purge flo is too fast.

7.2.1.5.2 Bromoform: This cor is one of the compounds most likely to be purged ve pd if the purge flow is too slow. Cold spots and $r$ actim ites in the transfer lines may adversely affert sponse.

7.2.1.5.3 Tetrachlore hane and 1,1-dichth th the: These compounds are degraded contaninated transfer wes in purge-and-trap systems vor actif sites in frapping materials.

7.2.1.6 The analytes in 8020 normally are not as negatively affected by small change, purge flow or system contamination. When analyzing for very eluting compounds with Method 8021 (i.e., s-trichlorobenzene, etc.), cross contani, and memory fects from a high concentration sample standard are a comon problem. Extra rinsing of the pur char malys is normally corrects this. Moisture effects re gen a ph lem with Method 8030 because of the high temperature ge. The newer purge-and-trap systems often ginne this prol em with better bakeout of the system purge-and-t p process. Also, the charcoal traps
sture and de rease the problem.

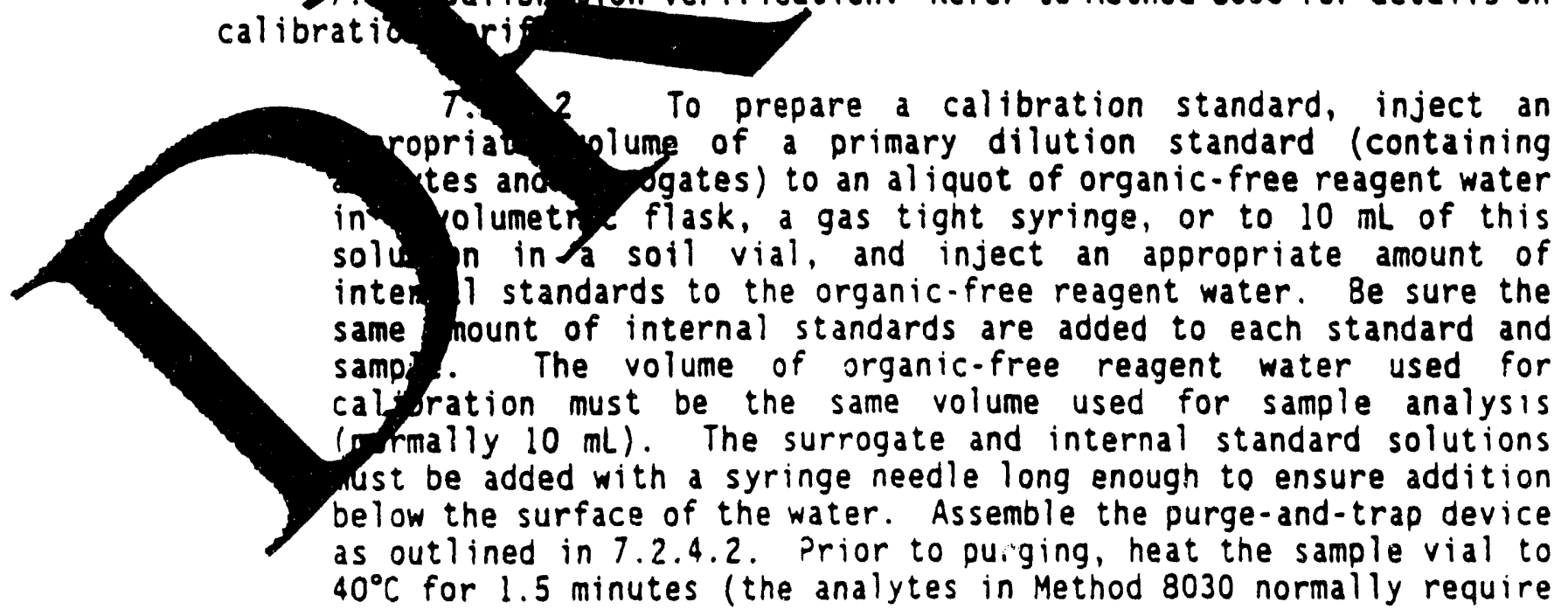


an $85^{\circ} \mathrm{C}$ purge temperature). Follow the guidance for the purge-andtrap procedure in section 7.2.4.4. GC or GC/MS calibration verification criteria must be met as specified in Method 8000 before analyzing samples.

NOTE: The device on the autosampler that introduces the solution containing the internal standards and surrogates must be disabled during calibration. Aqueous standards are not stable and should be discarded after one hour unless transferred to a sample bottle (or gas tight syringe) with no headspace and s immediately.

\subsubsection{Sample screening}

7.2.3.1 It is highly recommep. $t$. 11 samples be screened prior to the purge-and-trap GC $r$ GC/MS lysis. These samples may contain percent quantiti on purgeabl ganics that will contaminate the purge-and-t ap system ther requjing extensive cleanup and instrument owntime. See section or for suggested screening techniques ose the s ceening data to stermine whether to use the Low Concer. tion modj purge-and crap or to prepare samples by the High co. trati method.

7.2.3.2 Two suggested sc ing techniques are: the use of an automated headspace sampler in. ced to a gas chromatograph (GC) equipped with a photo ionizas detector (PID) and an electrolytic conduc aty detector (HECO) series; or, extraction of the sample with une (Method 3,0) and analysis of the extract on a GC equ pped EID and/or an ECD. Use the Low Concentration modifie purge the estimated concentration falls within the cal "at in rang of the selected determinative method. If the concen cion excreds the calibration range, then preparates by Concentration method (Section 7.3).

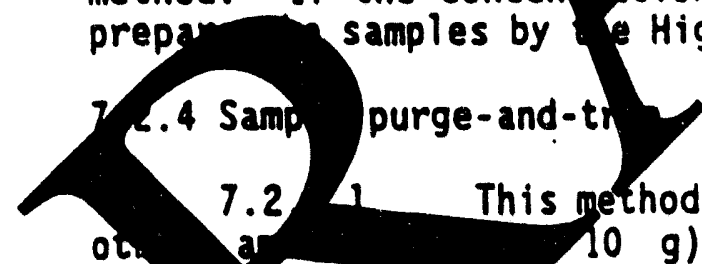
heriu. Gly seareu at the sampling site, and MUST remain so to

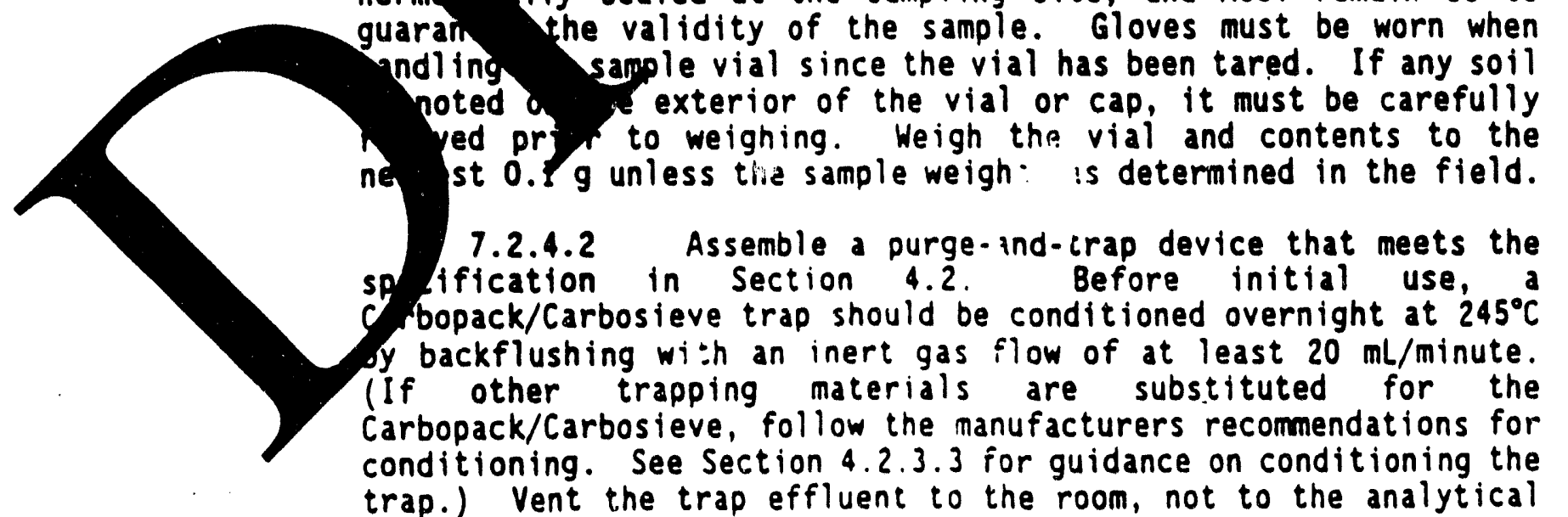




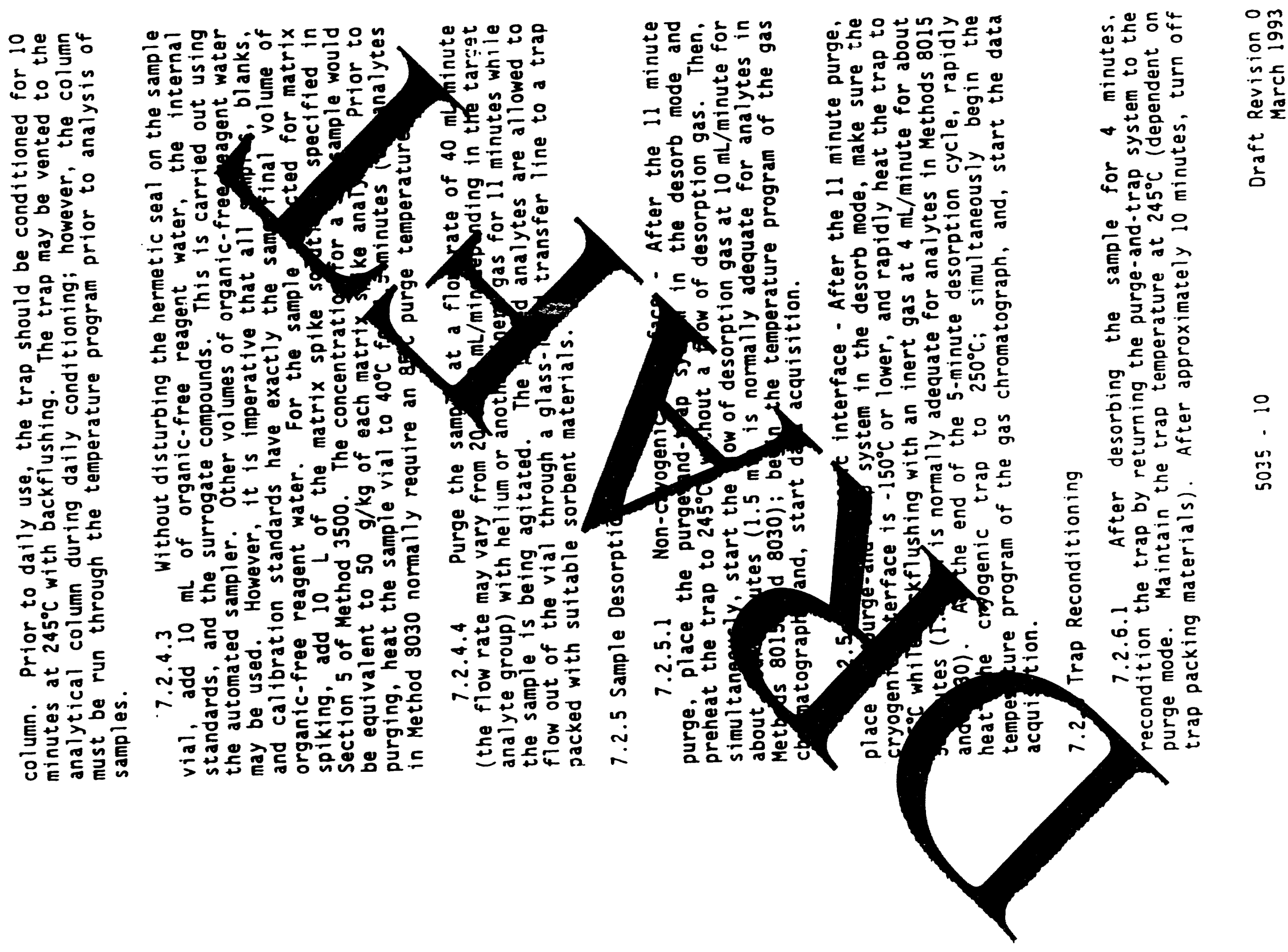


the trap heater and halt the purge flow through the trap. When the trap is cool, the next sample can be analyzed.

\subsubsection{Data Interpretation}

7.2.7.1 Perform qualitative and quantitative analysis on the data following the guidance given in the determinative method and Method 8000 . If concentrations of any target analyte exceeds the calibration range of the analyte, it will be necessary to reanalyze the sample by the Htgh Concentration Method.

\subsubsection{Determination of $\%$ Ory Weight}

7.2.8.1 Weigh 5-10 g of the sample into a tared crucible.

NOTE: It is highly recommended thet samples dry weight determination be withdra" an the $60 \mathrm{~mL}$ val until it is certain that no Mytical samples wh neded for High Concentrat 1 analys. This is to nimize loss of volatiles to avoid ple contaming ion from the laboratory

7.2.8.2 Determine the $\%$ d ight of the sample by drying overnight at $105^{\circ} \mathrm{C}$. Allow to cool th thiccator before weighing:

* dry weight $\frac{\text { of } \frac{d r y}{\text { sample }} \times}{\text { sumple }} x$<smiles>CC1C[C@H](C)[C@@H](C)C1</smiles>

HARNING: The trying be contained in a hood or vent 1 gnific Maboratory contamination may resui rom a bavily contaninated hazardous waste mple.

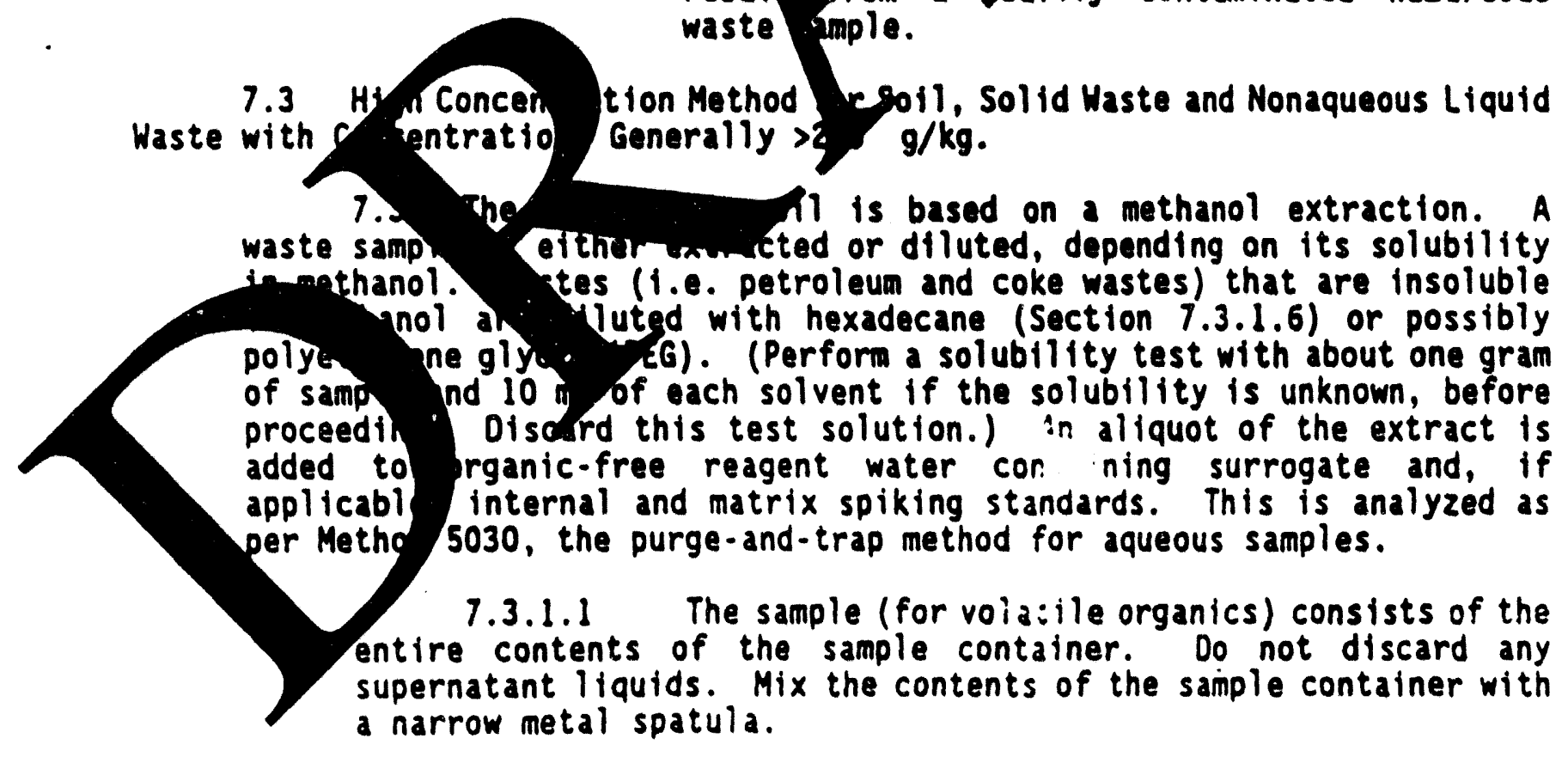


7.3.1.2 For soil and solid waste that is insoluble in methanol, weigh $\mathrm{g}$ (wet weight) of sample into a tared $20 \mathrm{~mL}$ vial. Use a top-loading balance. Note and record the actual weight to 0.1 gram and determine the percent dry weight of the sample using the procedure in Section 7.1.8. Quickly add $9.0 \mathrm{~mL}$ of methanol; then add $1.0 \mathrm{ml}$ of the surrogate spiking solution to the vial. Cap and shake for 2 min.

7.3.1.3 For waste that is soluble in methanol or PEG weigh $1 \mathrm{~g}$ (wet weight) into a tared scintillation vial or cylture tube or a $10 \mathrm{~mL}$ volumetric flask. (If a vial or tube is y it must be calibrated prior to use. Pipet $10.0 \mathrm{~mL}$ of meth of into the vial and mark the bottom of the meniscus. Disf 4 this solvent.) quickly add $1.0 \mathrm{~mL}$ of surrogate spiking sol th to the vial or flask and dilute to $10.0 \mathrm{~mL}$ with the appropp te sc. Shake the vial to mix the contents. For certain oif liquids, following methanol dilution/extraction has proventinctive. $g$ of olly liquid with $10 \mathrm{~mL}$ of methanol (2 Nnute shake) which. ults in the target analytes being extracted nto the methanol along the majority 0 . the oily waste (some the oil by still be flo ing on the surface). If oil is floatithe sur transfer to $2 \mathrm{~mL}$ to a clean GC vial using a Paste. ipet Ensure that no oil is transferred to the vial. Add 10 . of the methanol extract to $5 \mathrm{~mL}$ of organic-free reagent water purge-and-trap analysis. Prior to using this technique, test it poiking a $1 \mathrm{~g}$ aliquot of the ofly waste with matrix spike mix of the analytes of concern $(10$ - 50 b the matrix spike andard dissolved in methanol). Shake the inderspe the spike throughout the oll prior to addin the methanol extraction solvent. Compare the data with ingle wo of olly waste presented in Method 8260 . If recover is ot with the limits presented for the majority of compounds, the hexidecane dilution technique in Section 6 .

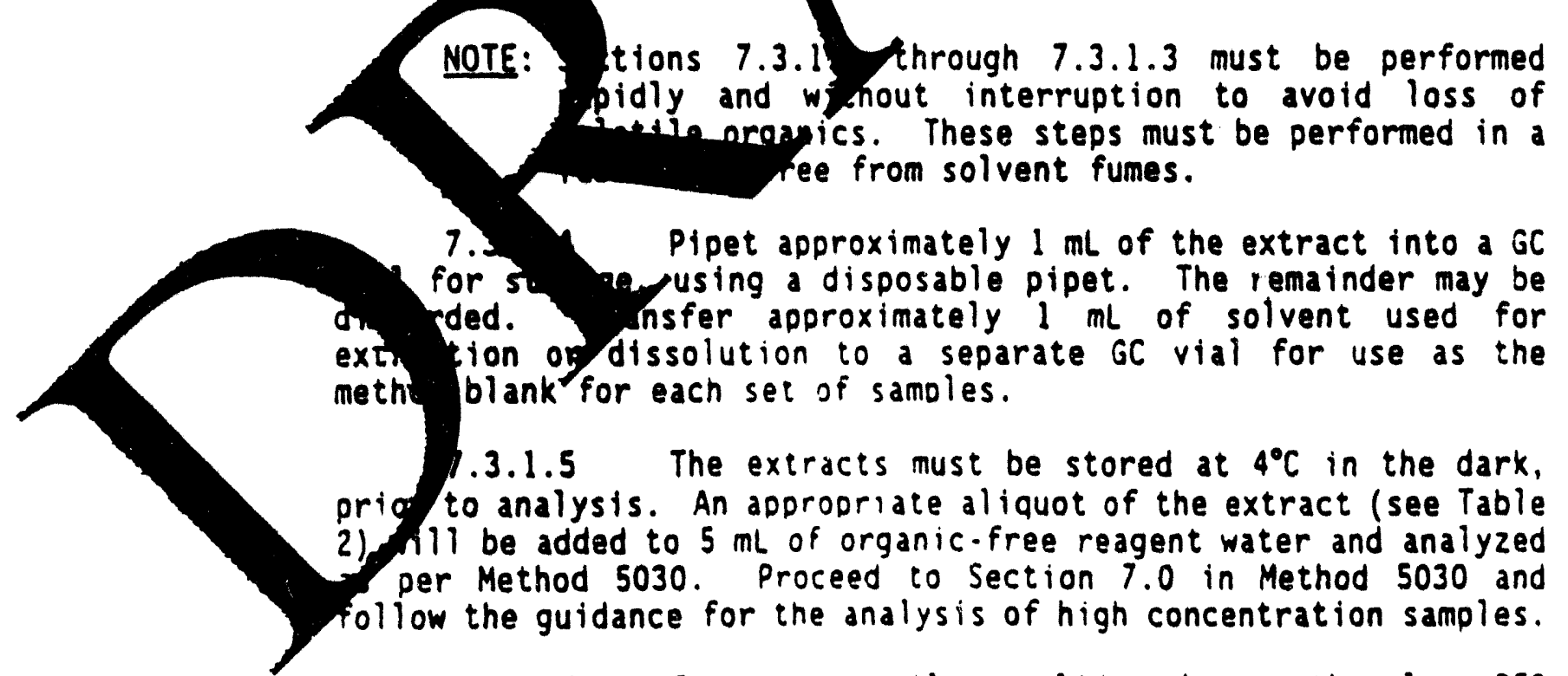

7.3.1.6 For wasie, soil or solids, where methanol or PEG are not effective solvents (e.g.. those jamples consisting primarily 
of petroleum or coking waste) dilute or extract with hexadecane following the guidance in Method 3585 (Waste Dilution for Volatiles).

\subsection{QUALITY CONTROL}

8.1 Refer to Chapter One for specific quality control procedures and Method 5000 for sample preparation QC procedures.

8.2 Before processing any samples, the analyst should demmstrate through the analysis of an organic-free reagent water method blank that lassware and reagents are interference free. Each time a set of sampl is extracted, or there is a change in reagents, a method blank should be pr ssed as a safeguard against chronic laboratory contamination. The blank s" ol hould be carried through all stages of the sample preparation and mear emen.

8.3 Standard quality assurance practices so used wh is method. Field duplicates should be collected to validat the precision of sampling techntque. Each analysis batch of 20 or les samples must contain. agent blank; either a matrix spike/matrix spike duplicat or a matrix ike and duplicate sample analysis; and a lab ry cont sample, wess the determinative method provides other guifania.

8.4 Surrogate standards should be added the appropriate determinative method.

\subsection{METHOD PERFORMANCE}

9.1 Single laboratory acco acy pin data were obtained for the method analytes in three soll matri ss and, a 11 collected 10 feet below the surface of a hazardous landftll, $c$ ad C-Horizon, and a surface garden soll. Each sample was an with the palytes at a concentration of $20 \mathrm{ng} / 5 \mathrm{~g}$, which is equivent $\mathrm{g} / \mathrm{kg}$. Thes data are listed in tables found in Method 8260 .

9.2

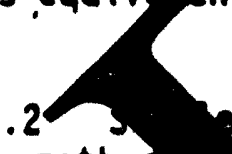

certain method.

12

ricy and

1 samples when specified in

extraction solve. comon spik

'acting oily liquid using methanol as the The data is presented in a table in Method 8260. The of oily liquit. worst set of data based on recovery data from many sources

9.3 Met detection limits (MDL) for soil were calculated by analyzing sand, the rix with the least matrix effec:. Replicate. $5 \mathrm{~g}$ samples were fo jed with $10 \mathrm{ng}$ of each of the method analytes. After an equilibration per aach imple was analyzed according to Section 11, and quantitated using fluor. as the internal standard. The results, in nanograms recovered from each 5 \& imple, are listed in Table 6. Using these data, Mols were calculated 


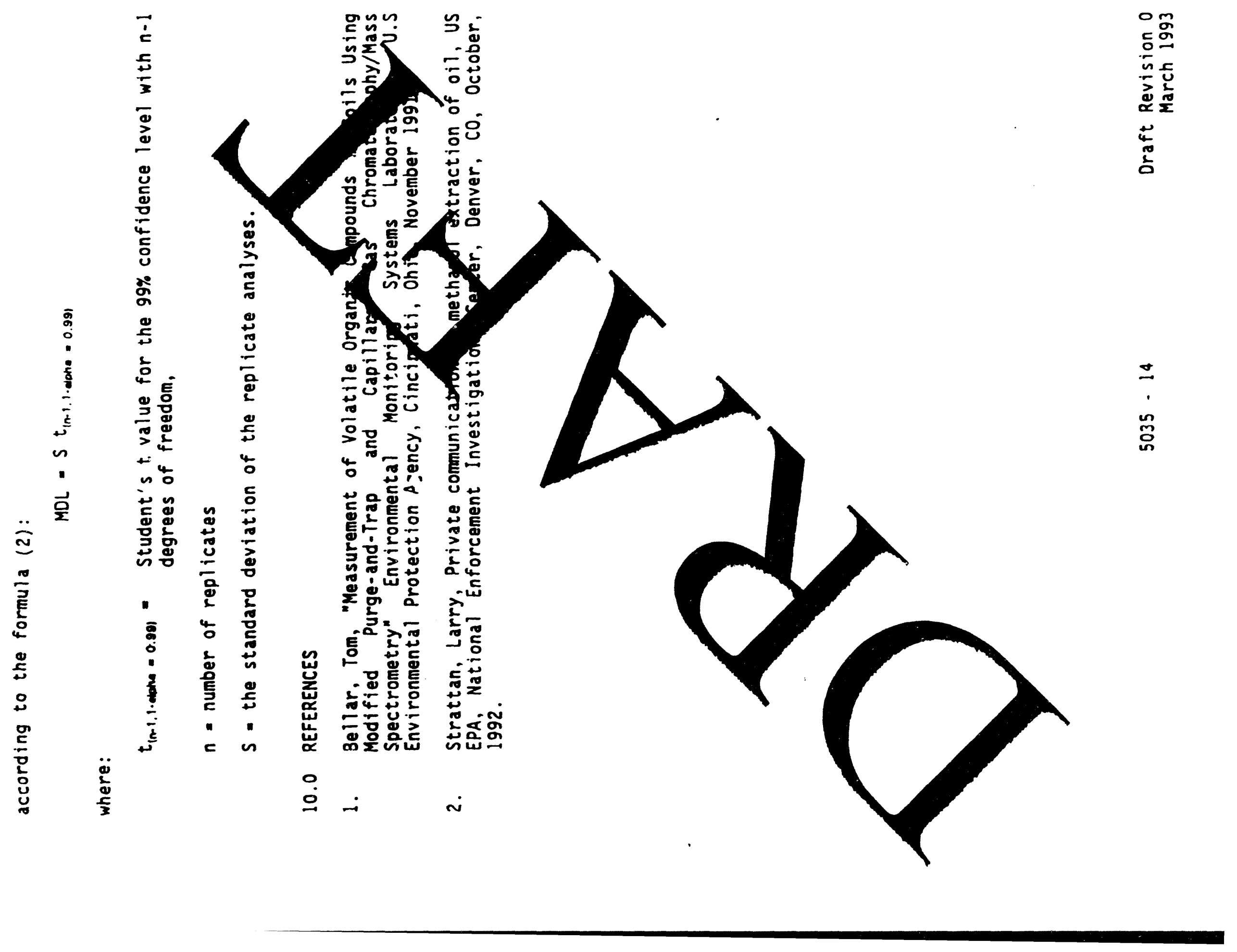


TABLE 1

DETERMINATIVE METHODS INTERFACED TO METHOD 5035

METHOD

METHOD NAME

8010

8015

8020

8021

8030

8240

8260

8266

Halogenated Volatile Organics

Nonhalogenated Volatile Organics Using GC/FID

Aromatic Volatile Organics by Gas Chromatography

Halogenated and Aromatic Volatiles by GC with Detectors in Series: Capillary Column

Acrolein and Acrylonitrile by Gas Chromato aphy

Volatile Organics by GC/MS: Packed Coly

Volatile Organics by GC/MS: Capillary

Volatile Organics by GC/MS: Capily y Co

with Isotope Dilution

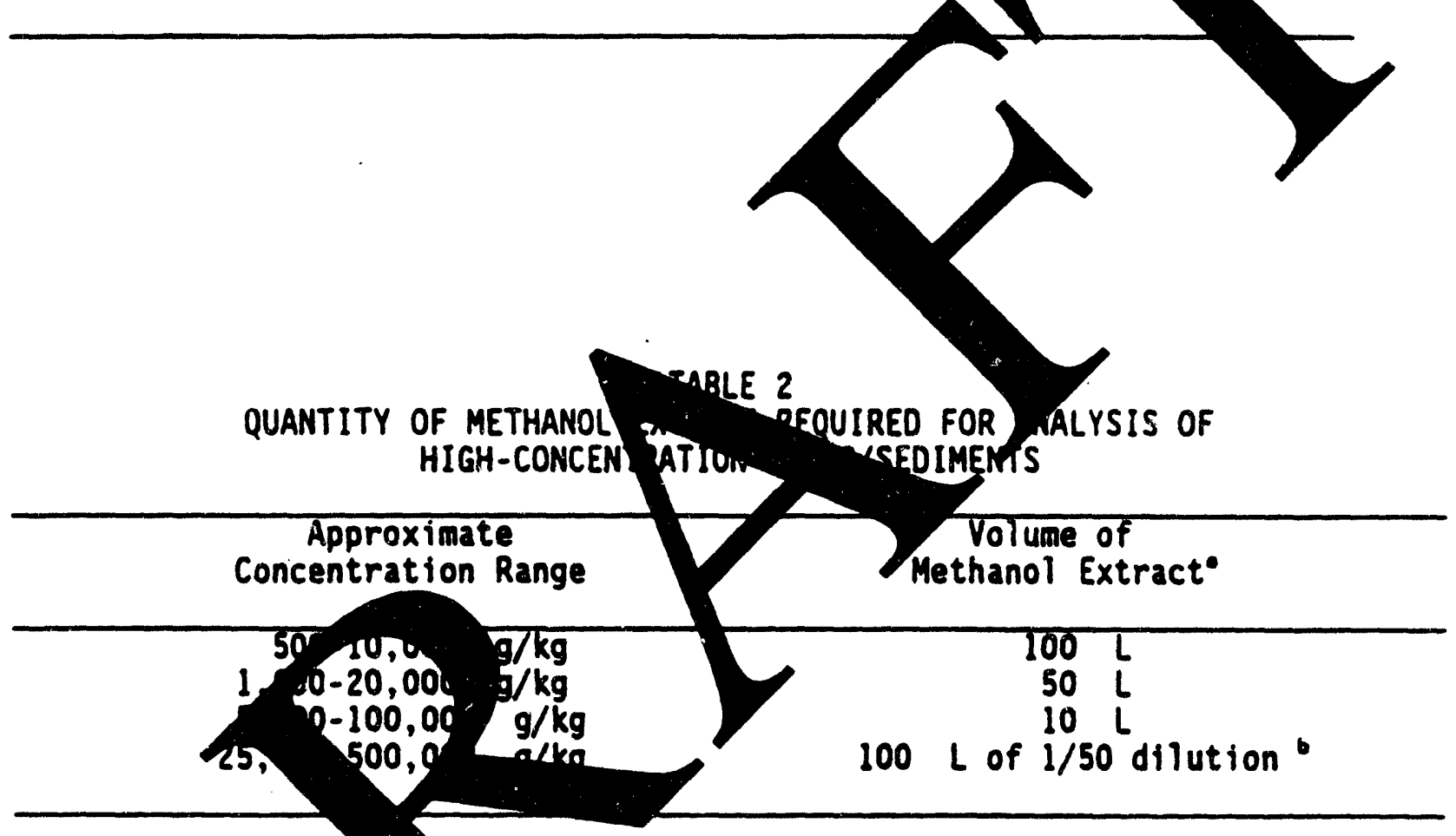

Calcul Yution factor for concentrations exceeding this table.

The vol of metlin added to $5 \mathrm{~mL}$ of water being purged should be kept constant. perefor, add to the $5 \mathrm{~mL}$ syringe whatever volume of methanol is necess to maintain a volume of $100 \mathrm{~L}$ added to the syringe.

Dilute an hiquot of the methanol extract and then take $100 \mathrm{~L}$ for palysis. 


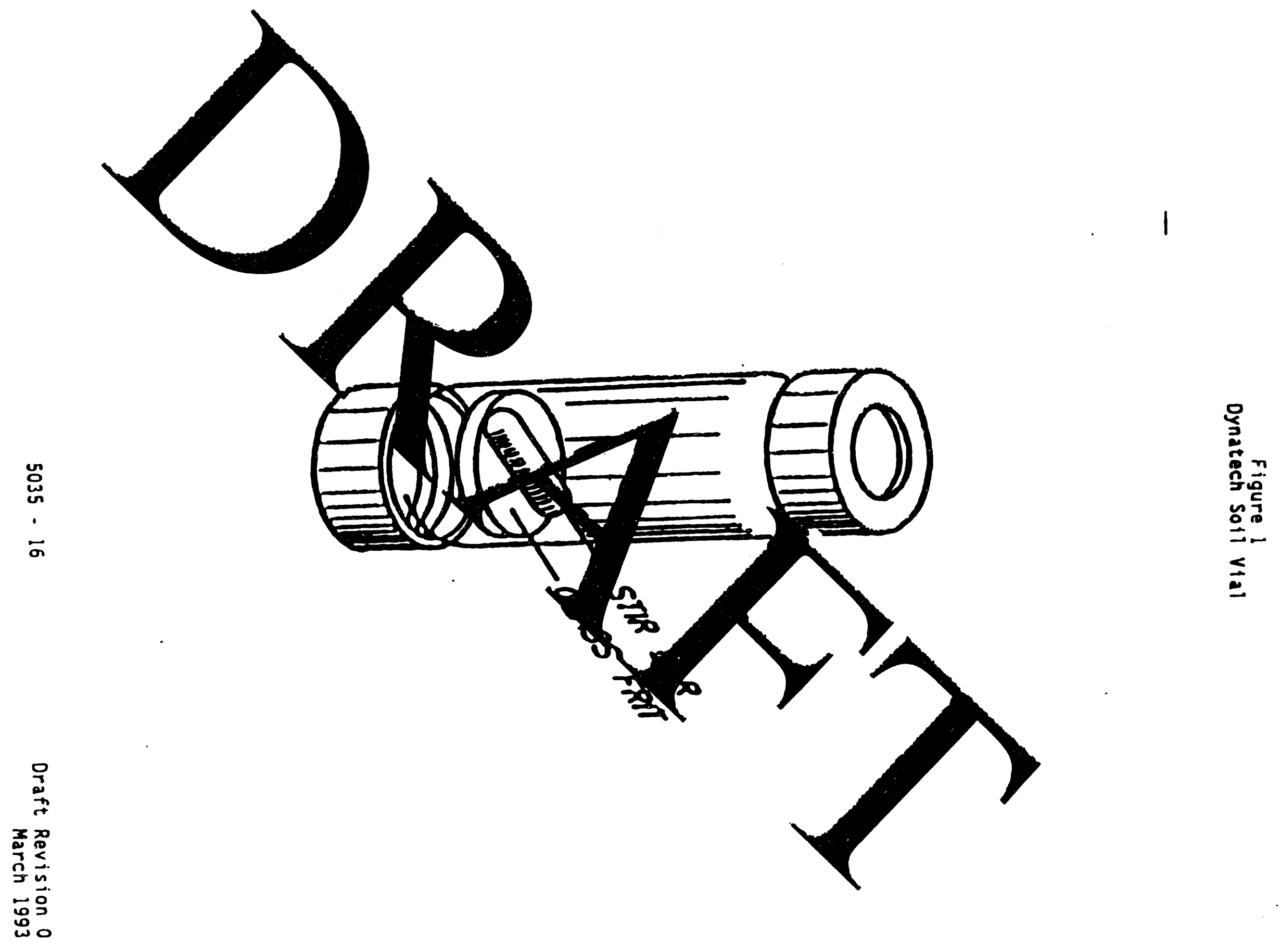




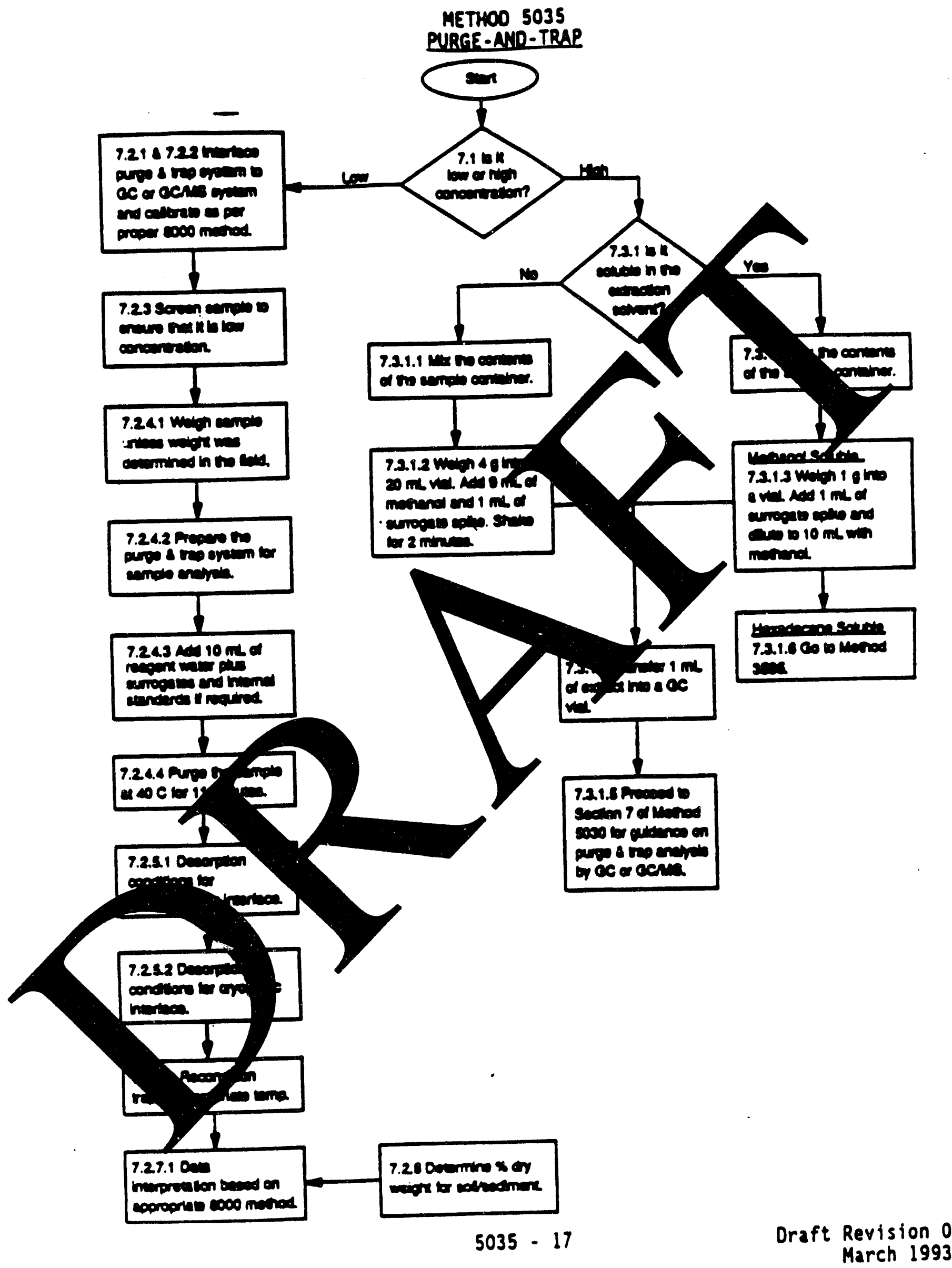


Attachment E - SRTC Calibration Curves and Response Data 


\section{Calibration Data for Depth Discrete Samples Collected from MBCSB-1}

Sample (sediment) concentrations are related to calibration concentrations using the equation:

Sediment concentration $[\mathrm{ug} / \mathrm{g}]=$

(Standard concentration [ug/L] x 0.007.5 [L]) / sediment mass [g]. 


\begin{tabular}{|c|c|c|c|c|c|c|}
\hline Samples & TCE area & PCE aren & \multicolumn{2}{|l|}{ Samp. Info } & $\mathrm{TCE}_{\mu \Omega} / \mathrm{L}$ & PCE $\mu \mathrm{g} / \mathrm{L}$ \\
\hline 0 & $0.000 E+\infty 0$ & $0.000 \mathrm{E}+00$ & \multicolumn{2}{|l|}{0} & 0.0 & 0.0 \\
\hline so & $5.091 E+06$ & $8.276 E+06$ & \multicolumn{2}{|c|}{ STANDARD TCE/PCE MDX } & 62.0 & 31.0 \\
\hline 100 & $1.101 E+07$ & $1.844 E+07$ & \multicolumn{2}{|c|}{ STANDARD TCE/PCE MDX } & 122.0 & 61.0 \\
\hline 150 & $2.063 E+07$ & $3.771 E+07$ & \multicolumn{2}{|c|}{ STANDARD TCE/PCE MDX } & 182.0 & 91.0 \\
\hline 200 & $2.849 E+07$ & $4.715 E+07$ & \multicolumn{2}{|c|}{ STANDARD TCE/PCE MDX } & 242.0 & 121.0 \\
\hline 250 & $3.608 E+07$ & $5.177 E+07$ & \multicolumn{2}{|c|}{ STANDARD TCE/PCE MDX } & 300.0 & 150.0 \\
\hline 300 & $5.375 E+07$ & $6.441 E+07$ & \multicolumn{2}{|c|}{ STANDARD TCE/PCE MIX } & 581.0 & 291.0 \\
\hline 750 & $6.114 E+07$ & $7.102 E+07$ & \multicolumn{2}{|c|}{ STANDARD TCE/PCE MDX } & 845.0 & 423.0 \\
\hline 1000 & $6.449 E+07$ & $7.431 E+07$ & \multicolumn{2}{|c|}{ STANDARD TCE/PCE MLX } & 1094.0 & 547.0 \\
\hline & TCE & & \multicolumn{3}{|c|}{ PCE } & \\
\hline slope & $1.127 E-05$ & & slope & $3.382 \mathrm{E}-06$ & & \\
\hline y intercept & $0.000 \mathrm{E}+00$ & & $y$ intercept & $0.000 E+00$ & & \\
\hline$\sqrt{2}$ & 0.99653 & & r2 & 0.96373 & & \\
\hline AREA & FIT VALUES & ACTUAL VALUES & AREA & FIT VALUES & ACTUAL VALUES & \\
\hline $0.000 E+\infty 0$ & 0.0 & 0.0 & $0.000 \mathrm{E}+00$ & 0.0 & 0.0 & \\
\hline $5.091 E+06$ & 57.4 & 62.0 & $8.276 E+06$ & 28.0 & 31.0 & \\
\hline $1.101 E+07$ & 124.1 & 122.0 & $1.844 E+07$ & 62.4 & 61.0 & \\
\hline $2.063 E+07$ & & 182.0 & $3.771 E+07$ & & 91.0 & \\
\hline $2.849 E+07$ & & 242.0 & $4.715 E+07$ & & 121.0 & \\
\hline $3.608 E+07$ & & 300.0 & $5.177 E+07$ & & 150.0 & \\
\hline $5.375 E+07$ & & 581.0 & $6.441 E+07$ & & 291.0 & \\
\hline $6.114 E+07$ & & 845.0 & $7.102 E+07$ & & 423.0 & \\
\hline $6.449 E+07$ & & 1094.0 & $7.431 E+07$ & & $\$ 47.0$ & \\
\hline
\end{tabular}




\section{MSB-CB। ECD/TCE}

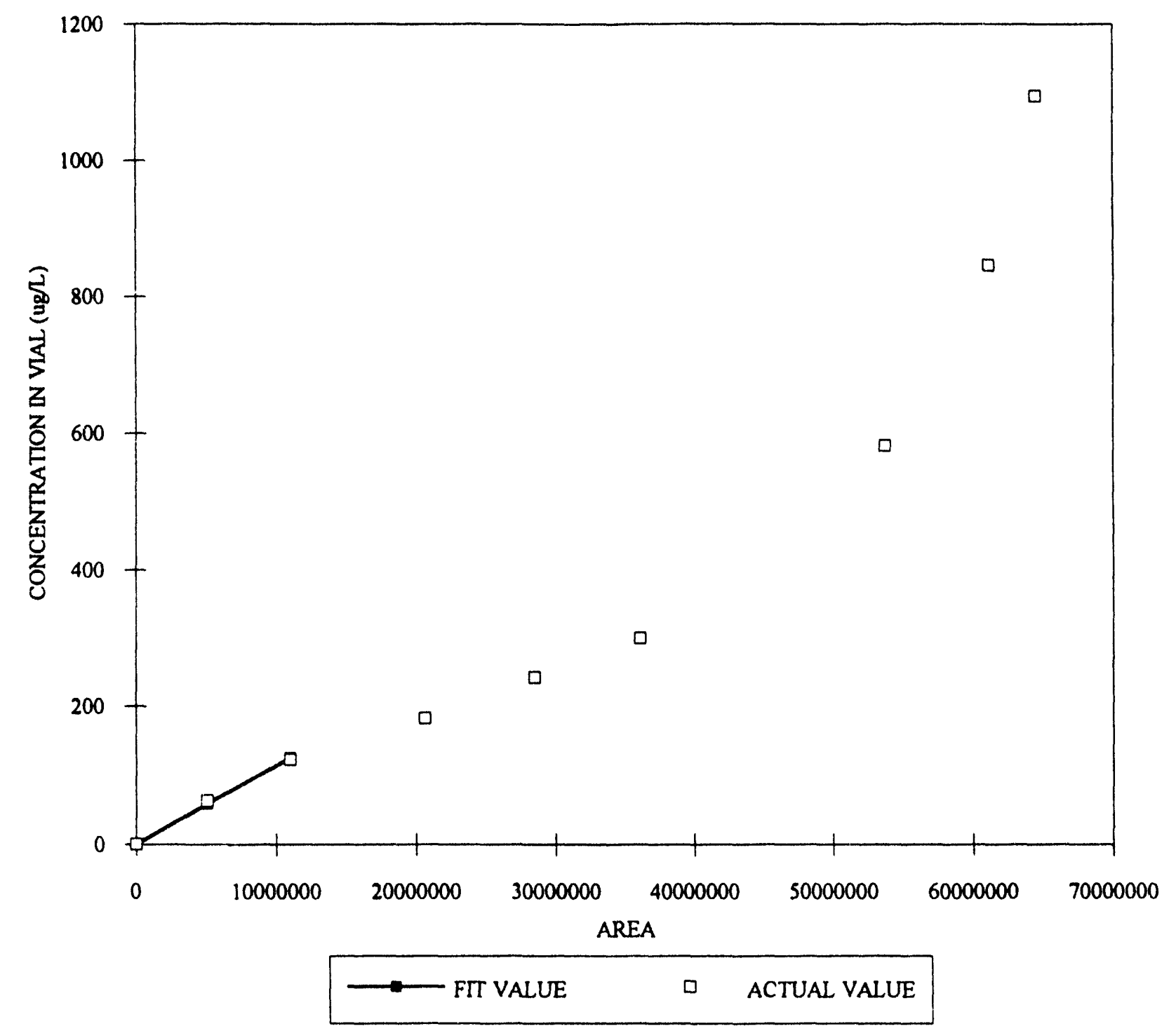


MSB-CB। ECD/PCE

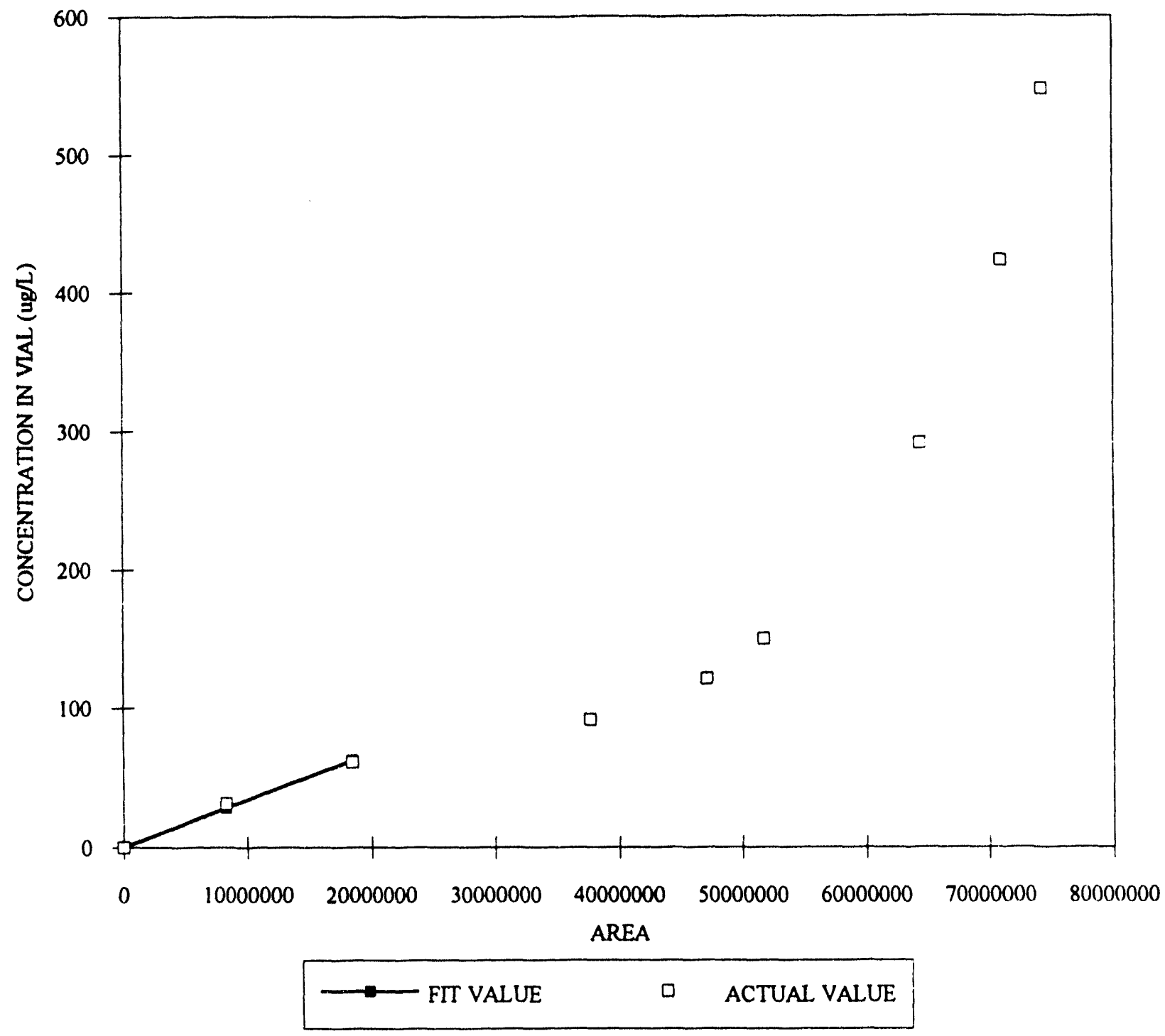




\section{Calibration Data for Depth Discrete Samples Collected from MBCSB-2}

Sample (sediment) concentrations are related to calibration concentrations using the equation:

Sediment concentration $[\mathrm{ug} / \mathrm{g}]=$

(Standard concentration [ug/L] x 0.0075 [L]) / sediment mass [g]. 
MSBCB2E.CAL

\begin{tabular}{|c|c|c|c|c|c|c|}
\hline Saniples & TCE area & PCE area & \multicolumn{2}{|l|}{ Semp. Info } & TCE $\mu g \Lambda$ & PCE $\mu \mathrm{R} / \mathrm{L}$ \\
\hline 0 & $3.196 E+03$ & $3.846 E+03$ & \multicolumn{2}{|l|}{0} & 0.0 & 0.0 \\
\hline so & $4.822 E+06$ & $7.795 E+06$ & \multicolumn{2}{|c|}{ STANDARD TCE/PCE MDX } & 62.0 & 31.0 \\
\hline 100 & $1.080 E+07$ & $1.803 E+07$ & \multicolumn{2}{|c|}{ STANDARD TCE/PCE MDX } & 122.0 & 61.0 \\
\hline 150 & $2.081 E+07$ & $3.819 E+07$ & \multicolumn{2}{|c|}{ STANDARD TCE/PCE MDX } & 182.0 & 91.0 \\
\hline 200 & $2.886 \mathrm{E}+07$ & $4.741 E+07$ & \multicolumn{2}{|c|}{ STANDARD TCE/PCE MDX } & 242.0 & 121.0 \\
\hline 250 & $3.769 E+07$ & $5.249 E+07$ & \multicolumn{2}{|c|}{ STANDARD TCE/PCE MDX } & 300.0 & 150.0 \\
\hline 500 & $3.438 E+07$ & $6.491 E+07$ & \multicolumn{2}{|c|}{ STANDARD TCE/PCE MD } & 581.0 & 291.0 \\
\hline 750 & $6.169 E+07$ & $7.146 E+07$ & \multicolumn{2}{|c|}{ STANDARD TCE/PCE MDX } & 845.0 & 423.0 \\
\hline \multirow[t]{2}{*}{1000} & $6.539 E+07$ & $7.547 E+07$ & \multicolumn{2}{|c|}{ STANDARD TCE/PCE MLX } & 1094.0 & 547.0 \\
\hline & TCE & & & PCE & & \\
\hline slope & 1.15SE-0S & & slope & $3.477 \mathrm{E}-06$ & & \\
\hline$y$ intercept & $0.000 E+00$ & & $y$ intercept & $0.000 \mathrm{E}+00$ & & \\
\hline $\mathbf{r}$ & 0.97357 & & $r^{2}$ & 0.99030 & & \\
\hline AREA & FIT VALUES & ACTUAL VALUES & AREA & FIT VALUES & ACTUAL VALUES & \\
\hline $3.196 \mathrm{E}+03$ & 0.0 & 0.0 & $5.846 \mathrm{E}+03$ & 0.0 & 0.0 & \\
\hline $4.822 E+06$ & 55.7 & 62.0 & $7.795 E+06$ & 27.1 & 31.0 & \\
\hline $1.080 E+07$ & 124.8 & 122.0 & $1.803 E+07$ & 62.7 & 61.0 & \\
\hline $2.081 E+07$ & & 182.0 & $3.819 E+07$ & 132.8 & & \\
\hline $2.886 E+07$ & & 242.0 & $4.741 E+07$ & 164.8 & & \\
\hline $3.769 E+07$ & & 300.0 & $5.249 E+07$ & 182.5 & & \\
\hline $3.438 E+07$ & & $\$ 81.0$ & $6.491 E+07$ & 225.7 & & \\
\hline $6.169 E+07$ & & 845.0 & $7.146 E+07$ & 248.4 & & \\
\hline $6.539 E+07$ & & 1094.0 & $7.547 E+07$ & 262.4 & & \\
\hline
\end{tabular}


MSB-CB2 ECD/TCE

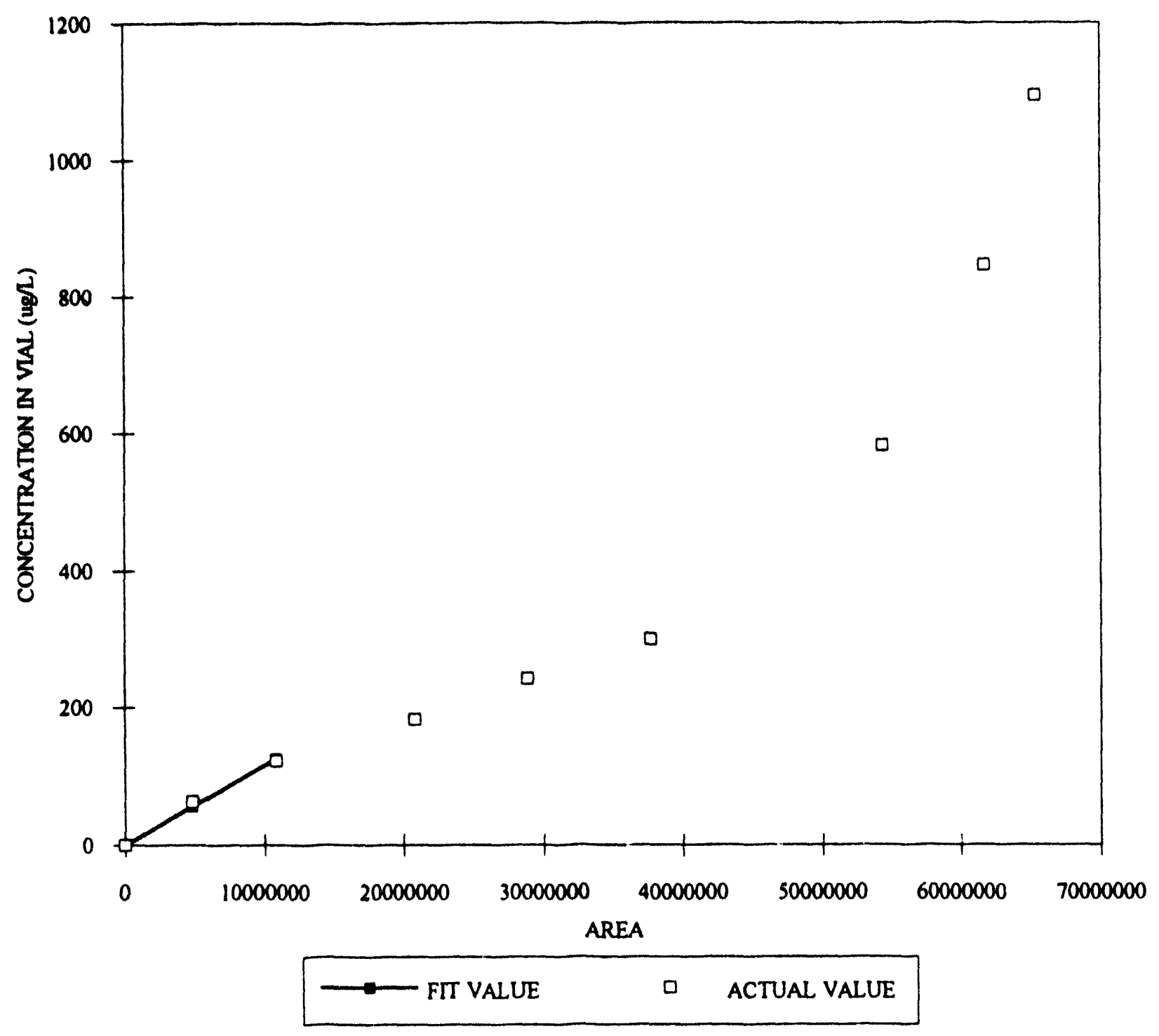


MSB-CB2 ECD/PCE

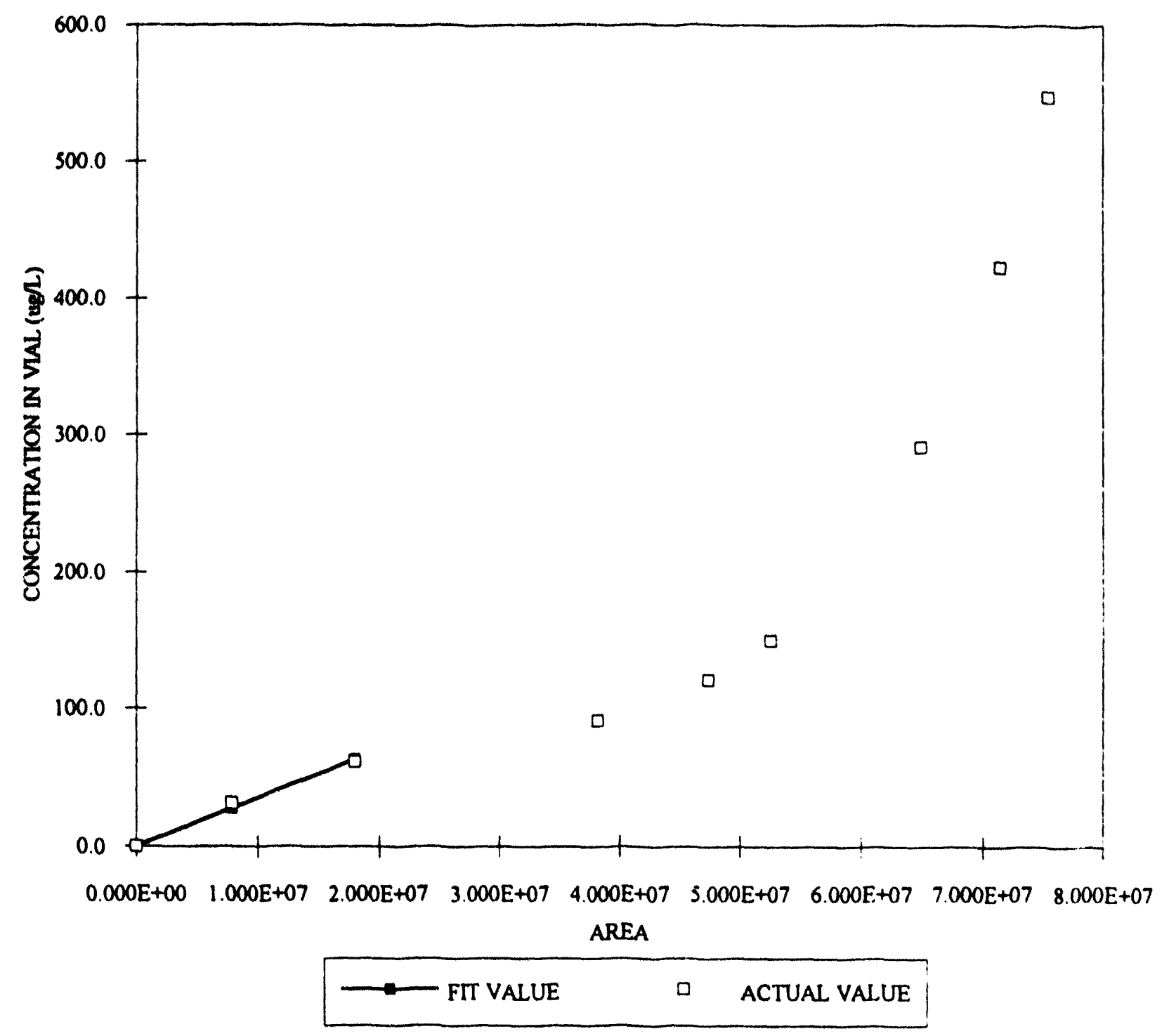




\section{Calibration Data for Depth Discrete Samples Collected from MBCSB-3}

Sample (sediment) concentrations are related to calibration concentrations using the equation:

Sediment concentration $[\mathrm{ug} / \mathrm{g}]=$

(Standard concentration [ug/L] x 0.0075 [L]) / sediment mass [g]. 


\begin{tabular}{|c|c|c|c|c|c|c|}
\hline Samples & TCE area & PCE area & \multicolumn{2}{|l|}{ Samp. Info } & TCE $\mu \Omega / L$ & PCE $\mu \mathrm{R} / \mathrm{L}$ \\
\hline 0 & $3.003 E+03$ & $2.195 E+03$ & \multicolumn{2}{|l|}{0} & 0.0 & 0.0 \\
\hline 25 & $2.941 E+06$ & 4.995E+06 & \multicolumn{2}{|c|}{ STANDARD TCE/PCE MDX } & 31.0 & 15.0 \\
\hline 100 & $1.347 E+07$ & $2.508 E+07$ & \multicolumn{2}{|c|}{ STANDARD TCE/PCE MDX } & 122.0 & 61.0 \\
\hline 200 & $3.011 E+07$ & $5.053 E+07$ & \multicolumn{2}{|c|}{ STANDARD TCE/PCE MDX } & 242.0 & 1210 \\
\hline 250 & $3.728 E+07$ & $5.429 E+07$ & \multicolumn{2}{|c|}{ STANDARD TCE/PCE MIX } & 300.0 & 150.0 \\
\hline 500 & $3.470 E+07$ & $6.666 E+07$ & \multicolumn{2}{|c|}{ STANDARD TCE/PCE MDX } & 581.0 & 291.0 \\
\hline 1000 & $6.621 E+07$ & $7.706 \mathrm{E}+07$ & \multicolumn{2}{|c|}{ STANDARD TCE/PCE MDX } & 1094.0 & $\$ 47.0$ \\
\hline & TCE & \multicolumn{5}{|c|}{ PCE } \\
\hline slope & $9.122 E-06$ & & slope & $2.454 E-06$ & & \\
\hline$y$ metercept & $0.000 \mathrm{E}+00$ & & $y$ intercept & $0.000 E+00$ & & \\
\hline+2 & 0.99773 & & $r 2$ & 0.99613 & & \\
\hline AREA & FIT VALUES & ACTUAL VALUES & AREA & FIT VALUES & ACTUAL VALLES & \\
\hline $3.005 E+03$ & 0.0 & 0.0 & $2.195 E+03$ & 0.0 & 0.0 & \\
\hline $2.941 E+06$ & 26.8 & 31.0 & 4.995E+06 & 12.3 & 15.0 & \\
\hline $1.347 E+07$ & 122.9 & 122.0 & $2.508 E+07$ & 61.5 & 61.0 & \\
\hline $3.011 E+07$ & & 242.0 & $5.053 E+07$ & & 121.0 & \\
\hline $3.728 E+07$ & & 300.0 & $5.429 E+07$ & & 150.0 & \\
\hline $3.470 E+07$ & & 581.0 & $6.666 \mathrm{E}+07$ & & 291.0 & \\
\hline $6.621 E+07$ & & 1094.0 & $7.706 E+07$ & & 547.0 & \\
\hline
\end{tabular}


MSB-CB3 ECD/TCE

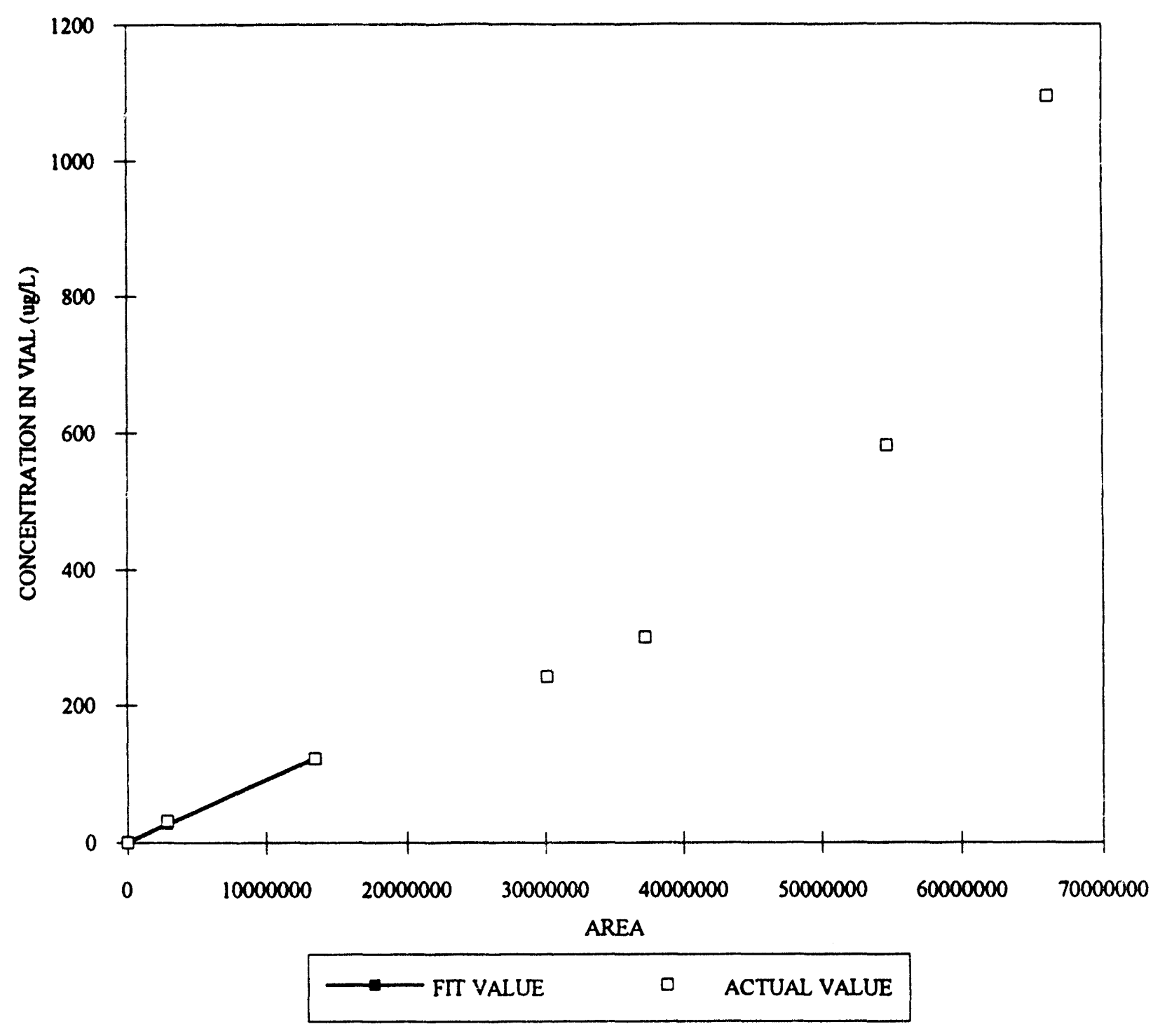


'.

MSB-CB3 ECD/PCE

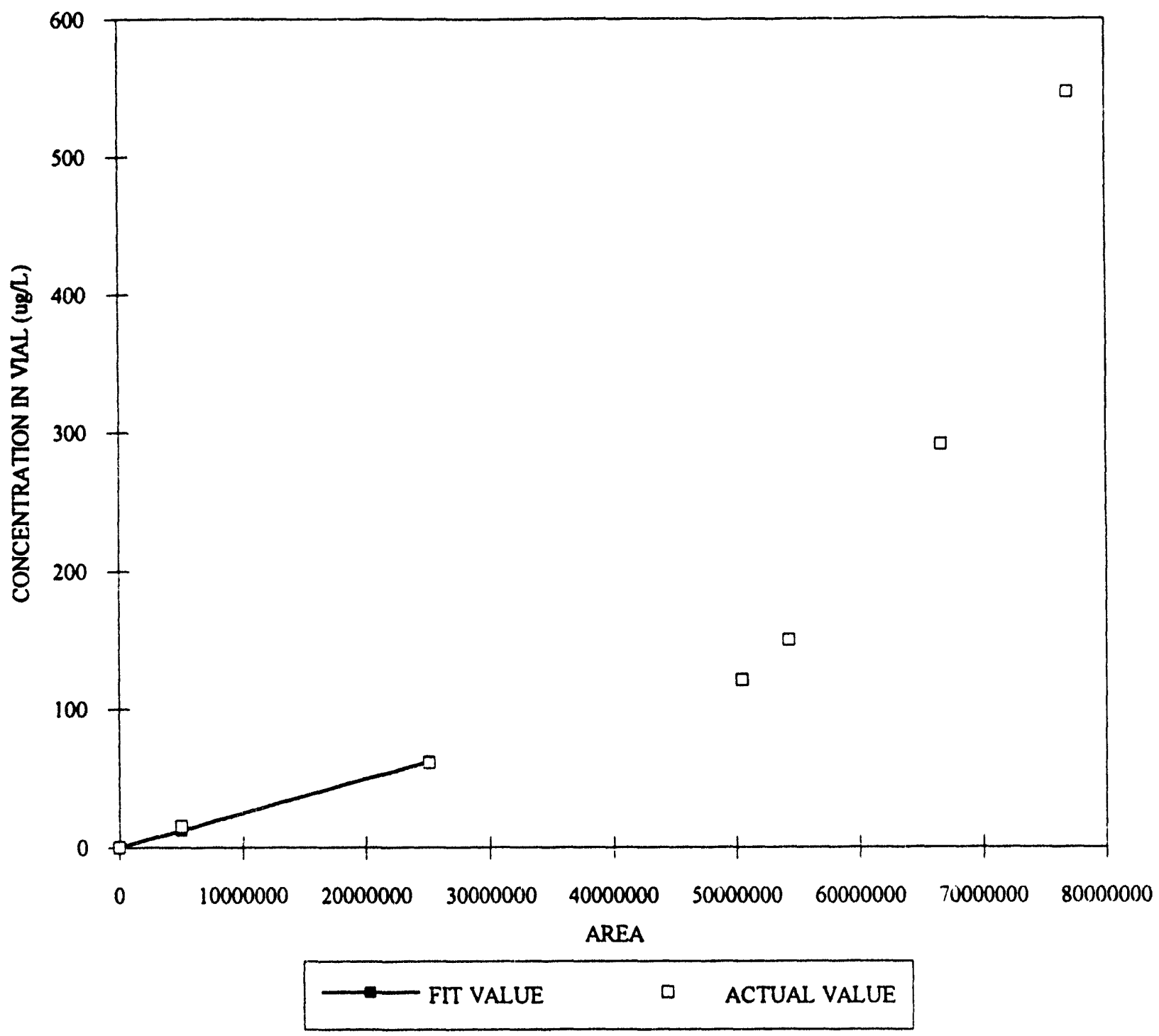


Calibration Data for Depth Discrete Samples Collected from MBCSB-4

Sample (sediment) concentrations are related to calibration concentrations using the equation:

Sediment concentration $[\mathrm{ug} / \mathrm{g}]=$

(Standard concentration [ug/L] x 0.0075 [L]) / sediment mass [g]. 
MSBCB4E.CAL

\begin{tabular}{|c|c|c|c|c|c|c|}
\hline Samples & TCE area & PCE area & Samp. Info & & $\mathrm{TCE} \mu \mathrm{g} / \mathrm{L}$ & PCE $\mu \mathrm{g} / \mathrm{L}$ \\
\hline 0 & $4.282 E+03$ & $1.877 E+03$ & 0 & & 0.0 & 0.0 \\
\hline 50 & $6.930 E+06$ & $1.138 E+07$ & STANDARD TCE & CE MDX & 62.0 & 31.0 \\
\hline 100 & $1.308 E+07$ & $2.253 E+07$ & STANDARD TCE & CE MDX & 122.0 & 61.0 \\
\hline 150 & 2.395E+07 & 4.335E+07 & STANDARD TCE & CE MLX & 182.0 & 91.0 \\
\hline 200 & $3.089 E+07$ & $4.930 \mathrm{E}+07$ & STANDARD TCI & PCE MIX & 242.0 & 121.0 \\
\hline 250 & $3.723 E+07$ & $5.252 E+07$ & STANDARD TCI & PCE MIX & 300.0 & 150.0 \\
\hline 500 & $3.460 E+07$ & $6.518 \mathrm{E}+07$ & STANDARD TC & PCE MLX & 581.0 & 291.0 \\
\hline 750 & $6.166 E+07$ & $7.160 E+07$ & STANDARD TC & CE MDX & 845.0 & 423.0 \\
\hline 1000 & $6.532 \mathrm{E}+07$ & $7.507 E+07$ & STANDARD TCI & PCE MIX & 1094.0 & 547.0 \\
\hline & TCE & & & PCE & & \\
\hline slope & $7.995 E-06$ & & slope & $2.711 E-06$ & & \\
\hline$y$ intercept & $0.000 \mathrm{E}+00$ & & y intercept & $0.000 E+00$ & & \\
\hline $\mathbf{r}$ & 0.99928 & & $r 2$ & 0.99998 & & \\
\hline AREA & FIT VALUES & ACTUAL VALUES & AREA & FIT VALUES & ACTUAL VALUES & \\
\hline $4.282 E+03$ & 0.0 & 0.0 & $1.877 E+03$ & 0.0 & 0.0 & \\
\hline $6.930 E+06$ & 55.4 & 62.0 & $1.138 E+07$ & 30.8 & 31.0 & \\
\hline $1.308 E+07$ & 104.6 & 122.0 & $2.253 \mathrm{E}+07$ & 61.1 & 61.0 & \\
\hline $2.395 E+07$ & & 182.0 & $4.335 E+07$ & & 91.0 & \\
\hline $3.089 E+07$ & & 242.0 & $4.930 \mathrm{E}+07$ & & 121.0 & \\
\hline $3.723 E+07$ & & 300.0 & $5.252 E+07$ & & 150.0 & \\
\hline $5.460 E+07$ & & 581.0 & $6.518 \mathrm{E}+07$ & & 291.0 & \\
\hline $6.166 E+07$ & & 845.0 & $7.160 E+07$ & & 423.0 & \\
\hline $6.532 E+07$ & & 1094.0 & $7.507 E+07$ & & 547.0 & \\
\hline
\end{tabular}


MSB-CB4 ECD/TCE

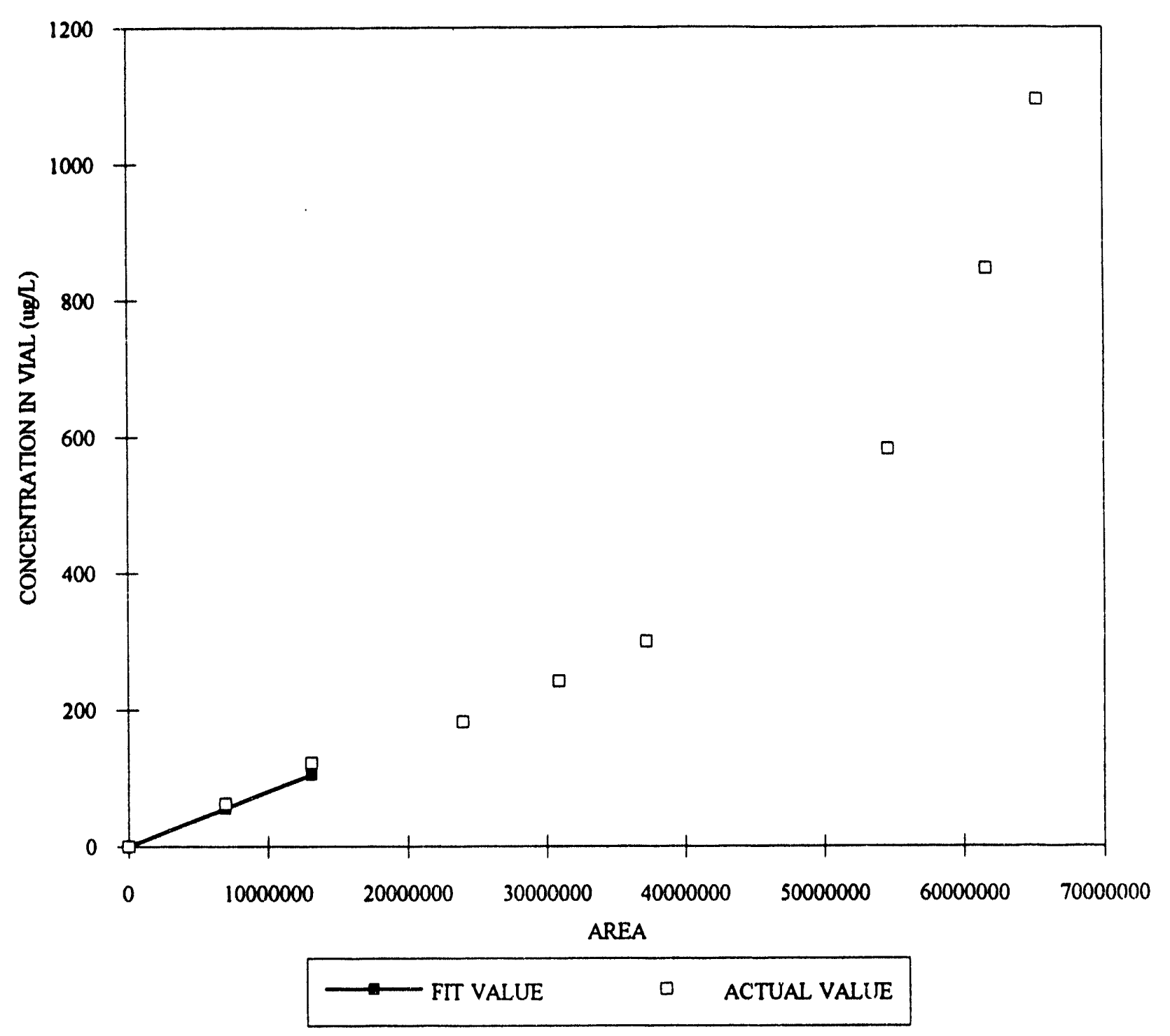


MSB-CB4 ECD/PCE

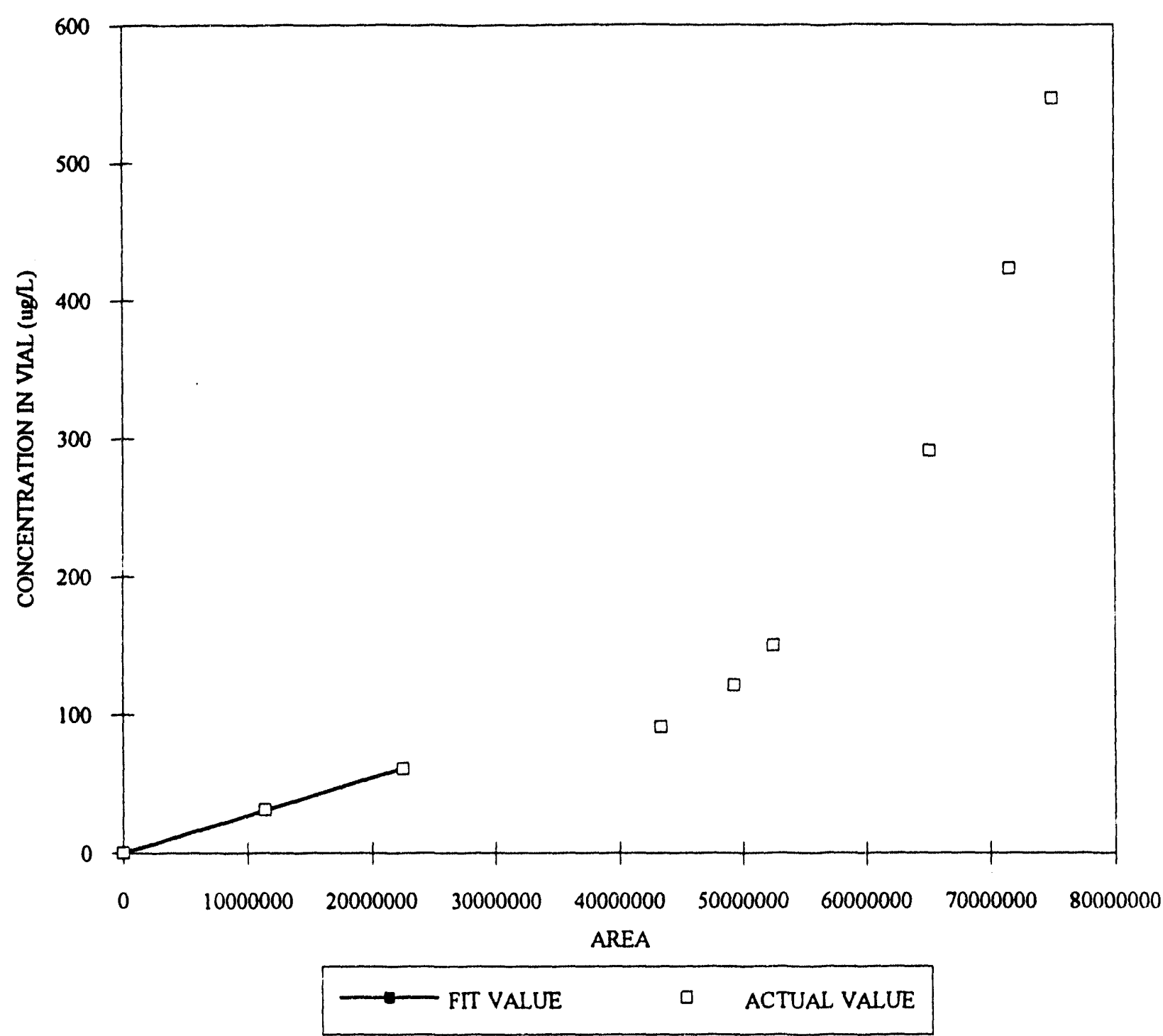




\section{Calibration Data for Depth Discrete Samples Collected from MBCSB-5}

Sample (sediment) concentrations are related to calibration concentrations using the equation:

Sediment concentration $[\mathrm{ug} / \mathrm{g}]=$

(Standard concentration $[\mathrm{ug} / \mathrm{L}] \times 0.0075[[\mathrm{~L}]) /$ sediment mass $[\mathrm{g}]$. 
MSBCB5E.CAL

\begin{tabular}{|c|c|c|c|c|c|c|}
\hline Samples & TCE area & PCE area & Samp. Info & & TCE $\mu g / L$ & $\mathrm{PCE}_{\mu \mathrm{g} / \mathrm{L}}$ \\
\hline 0 & $4.034 E+03$ & $2.640 E+03$ & 0 & & 0.0 & 0.0 \\
\hline so & $5.340 \mathrm{E}+06$ & $9.033 E+06$ & STANDARD TCE & CE MDX & 62.0 & 31.0 \\
\hline 100 & $1.124 \mathrm{E}+07$ & $1.965 E+07$ & STANDARD TCE & CE MLX & 122.0 & 61.0 \\
\hline 150 & $2.201 E+07$ & $4.161 E+07$ & STANDARD TCE & PE MDX & 182.0 & 91.0 \\
\hline 200 & $3.029 E+07$ & $4.940 E+07$ & STANDARD TCE & PCE MDX & 242.0 & 121.0 \\
\hline 250 & $3.895 E+07$ & $5.400 E+07$ & STANDARD TCE & PCE MLX & 300.0 & 150.0 \\
\hline 500 & $5.508 E+07$ & $6.633 E+07$ & STANDARD TCI & PCE MLX & 581.0 & 291.0 \\
\hline 750 & $6.248 E+07$ & $7.287 E+07$ & STANDARD TCI & PCE MDX & 845.0 & 423.0 \\
\hline \multirow[t]{2}{*}{1000} & $6.646 \mathrm{E}+07$ & $7.698 \mathrm{E}+07$ & STANDARD TCI & PCE MDX & 1094.0 & 547.0 \\
\hline & TCE & & & PCE & & \\
\hline slope & $1.099 \mathrm{E}-05$ & & slope & $3.162 \mathrm{E}-06$ & & \\
\hline$y$ intercept & $0.000 E+00$ & & $y$ intercept & $0.000 \mathrm{E}+00$ & & \\
\hline$r^{2}$ & 0.99821 & & $\mathbf{r}$ & 0.99613 & & \\
\hline AREA & FIT VALUES & ACTUAL VALUES & AREA & FIT VALUES & ACTUAL VALUES & \\
\hline $4.034 E+03$ & 0.0 & 0.0 & $2.640 E+03$ & 0.0 & 0.0 & \\
\hline $\begin{array}{l}5.340 E+06 \\
1.124 E+07\end{array}$ & 58.7 & 62.0 & $9.033 \mathrm{E}+06$ & 28.6 & 31.0 & \\
\hline $\begin{array}{l}1.124 E+07 \\
2.201 E+07\end{array}$ & 123.6 & 122.0 & $1.965 E+07$ & 62.1 & 61.0 & \\
\hline $\begin{array}{l}2.201 E+07 \\
3.029 E+07\end{array}$ & & 182.0 & $4.161 E+07$ & & 91.0 & \\
\hline $\begin{array}{l}3.029 E+07 \\
3.895 E+07\end{array}$ & & 242.0 & $4.940 \mathrm{E}+07$ & & 121.0 & \\
\hline $\begin{array}{l}3.895 E+07 \\
5.508 E+07\end{array}$ & & 300.0 & $5.400 E+07$ & & 150.0 & \\
\hline $\begin{array}{l}5.508 E+07 \\
6.248 E+07\end{array}$ & & 581.0 & $6.633 \mathrm{E}+07$ & & 391.0 & \\
\hline $\begin{array}{l}6.248 E+07 \\
6.646 E+07\end{array}$ & & 845.0 & $7.287 E+07$ & & 423.0 & \\
\hline & & 1094.0 & $7.698 E+07$ & & 547.0 & \\
\hline
\end{tabular}


MSB-CBS ECD/TCE

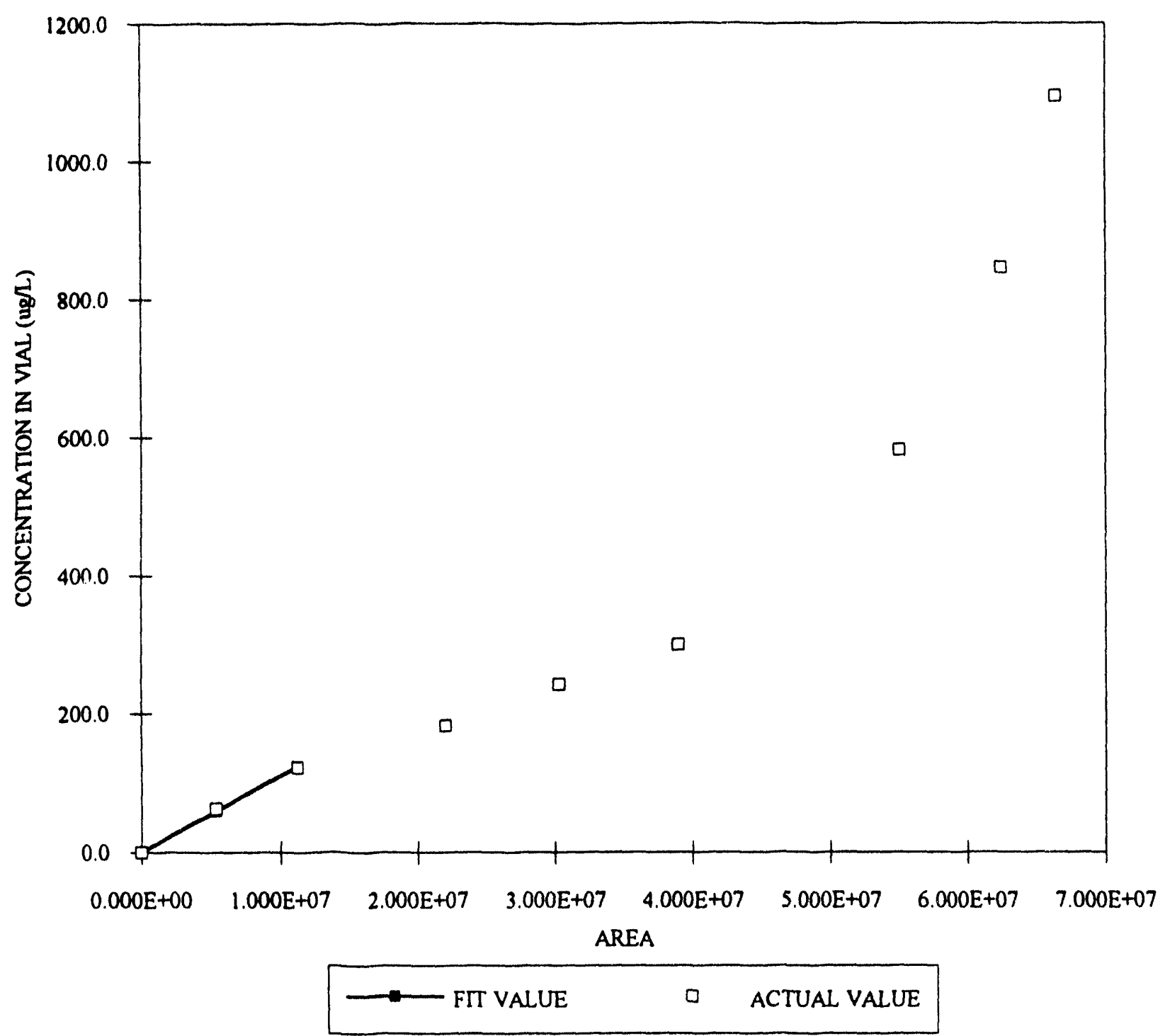


.91

MSB-CBS ECD/PCE

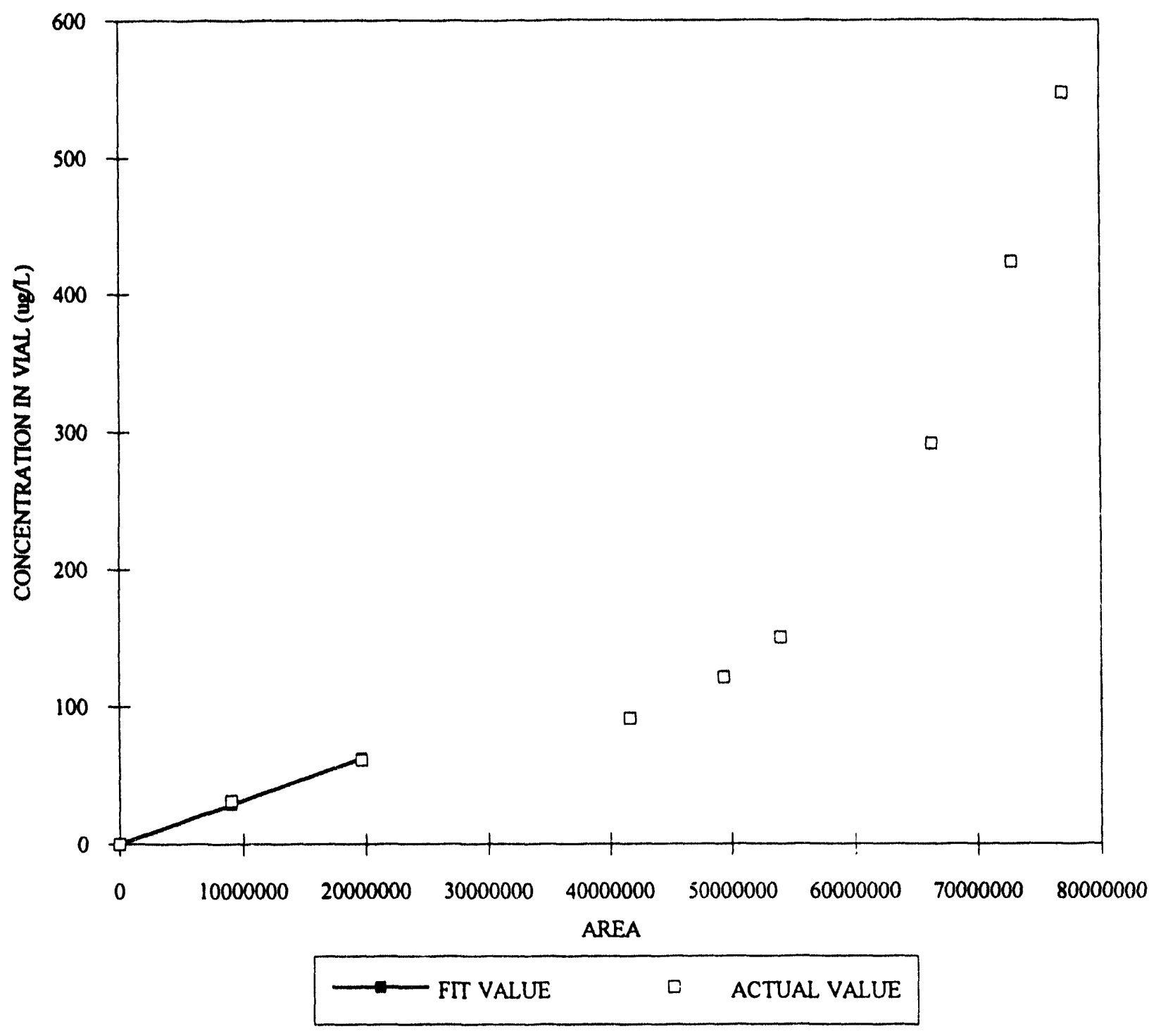




\section{Calibration Data for Depth Discrete Samples Collected from MBCSB-6}

Sample (sediment) concentrations are related to calibration concentrations using the equation:

Sediment concentration $[\mathrm{ug} / \mathrm{g}]=$

(Standard concentration [ug/L] $\times 0.0075[\mathrm{~L}]$ ) / sediment mass [g]. 
MSBCB6E.CAL

\begin{tabular}{|c|c|c|c|c|c|c|}
\hline Semples & TCE aren & PCE area & \multicolumn{2}{|l|}{ Samp. Info } & TCE MRR & PCE $\mu \mathrm{g} / \mathrm{L}$ \\
\hline 0 & $3.220 E+03$ & $4.047 E+03$ & \multicolumn{2}{|l|}{0} & 0.0 & 0.0 \\
\hline so & $9.117 E+06$ & $8.581 E+06$ & \multicolumn{2}{|c|}{ STANDARD TCE/PCE MDX } & 62.0 & 31.0 \\
\hline 100 & $1.105 \mathrm{E}+07$ & $1.916 \mathrm{E}+07$ & \multicolumn{2}{|c|}{ STANDARD TCE/PCE MDX } & 122.0 & 61.0 \\
\hline 150 & $2.159 E+07$ & $4.093 E+07$ & \multicolumn{2}{|c|}{ STANDARD TCE/PCE MDX } & 182.0 & 91.0 \\
\hline 200 & $2.941 E+07$ & $4.872 E+07$ & \multicolumn{2}{|c|}{ STANDARD TCE/PCE MDX } & 242.0 & 121.0 \\
\hline 250 & $3.842 E+07$ & $5.383 E+07$ & \multicolumn{2}{|c|}{ STANDARD TCE/PCE MDX } & 300.0 & 150.0 \\
\hline 300 & $3.475 E+07$ & $6.598 E+07$ & \multicolumn{2}{|c|}{ STANDARD TCE/PCE MDX } & 981.0 & 291.0 \\
\hline 750 & $6.249 E+07$ & $7.283 E+07$ & \multicolumn{2}{|c|}{ STANDARD TCE/PCE MDX } & 843.0 & 423.0 \\
\hline \multirow[t]{2}{*}{1000} & $6.628 E+07$ & $7.674 E+07$ & \multicolumn{2}{|c|}{ STANDARD TCE/PCE MDX } & 1094.0 & 547.0 \\
\hline & TCE & & & PCE & & \\
\hline slope & $1.123 E-03$ & & slope & $3.236 E-06$ & & \\
\hline y intercept & $0.000 E+00$ & & y intercept & $0.000 E+00$ & & \\
\hline r2 & 0.99666 & & $\mathbf{r}$ & 0.99395 & & \\
\hline AREA & FIT VALUES & ACTUAL VALUES & AREA & FIT VALUES & ACTUAL VALUES & \\
\hline $3.220 \mathrm{E}+03$ & 0.0 & 0.0 & $4.047 E+03$ & 0.0 & 0.0 & \\
\hline $9.117 E+06$ & 57.5 & 62.0 & $8.981 E+06$ & 27.9 & 31.0 & \\
\hline $1.105 E+07$ & 124.1 & 122.0 & $1.916 E+07$ & 62.4 & 61.0 & \\
\hline $2.159 E+07$ & & 182.0 & $4.093 E+07$ & & 91.0 & \\
\hline $2.941 E+07$ & & 242.0 & $4.872 E+07$ & & 121.0 & \\
\hline $3.842 E+07$ & & 300.0 & $3.383 E+07$ & & 150.0 & \\
\hline $5.475 E+07$ & & 581.0 & $6.598 E+07$ & & 291.0 & \\
\hline $6.249 E+07$ & & 845.0 & $7.283 E+07$ & & 423.0 & \\
\hline $6.628 E+07$ & & 1094.0 & $7.674 E+07$ & & $\$ 47.0$ & \\
\hline
\end{tabular}


MSB-CB6 ECD/TCE

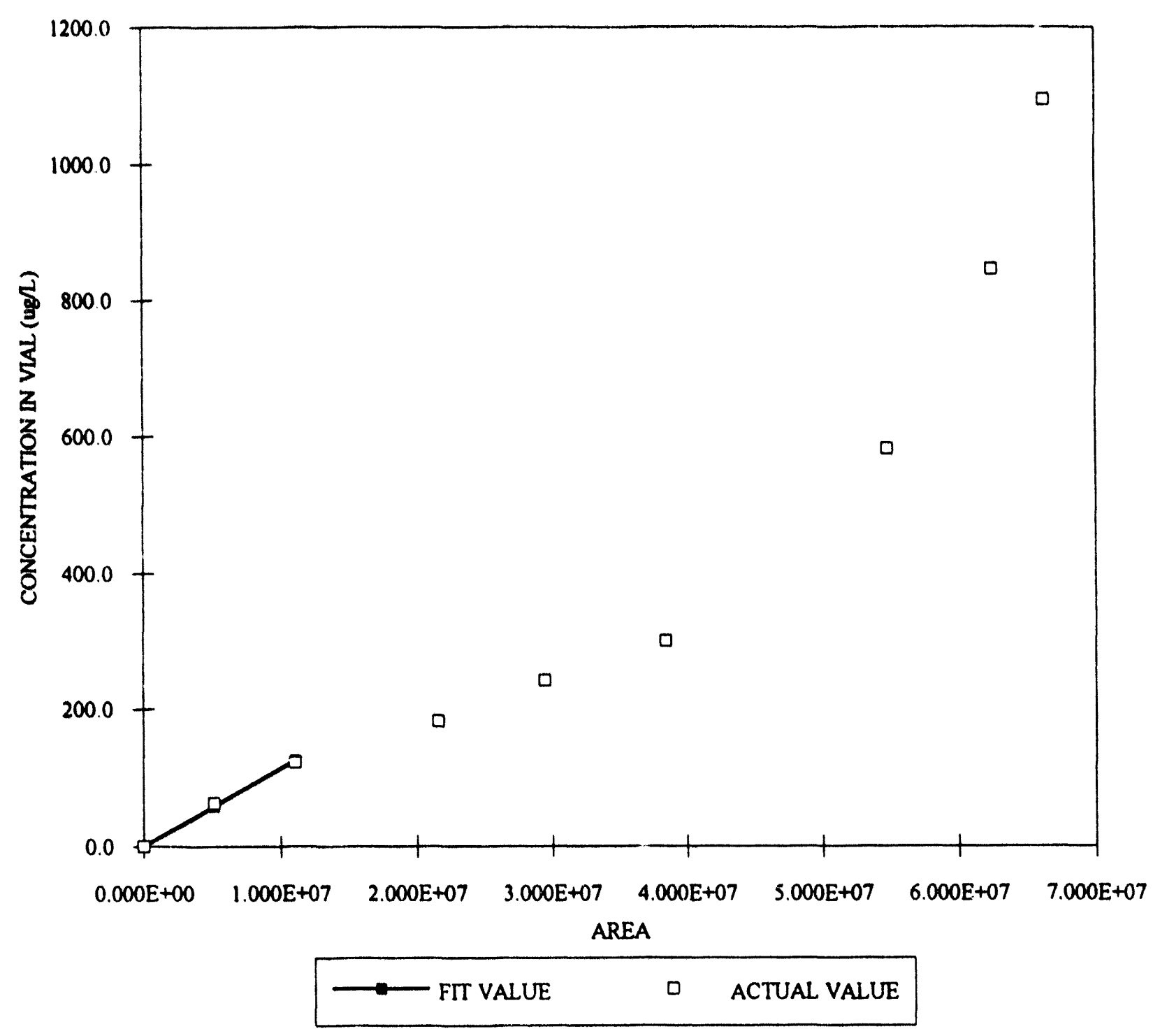


MSB-CB6 ECD/PCE

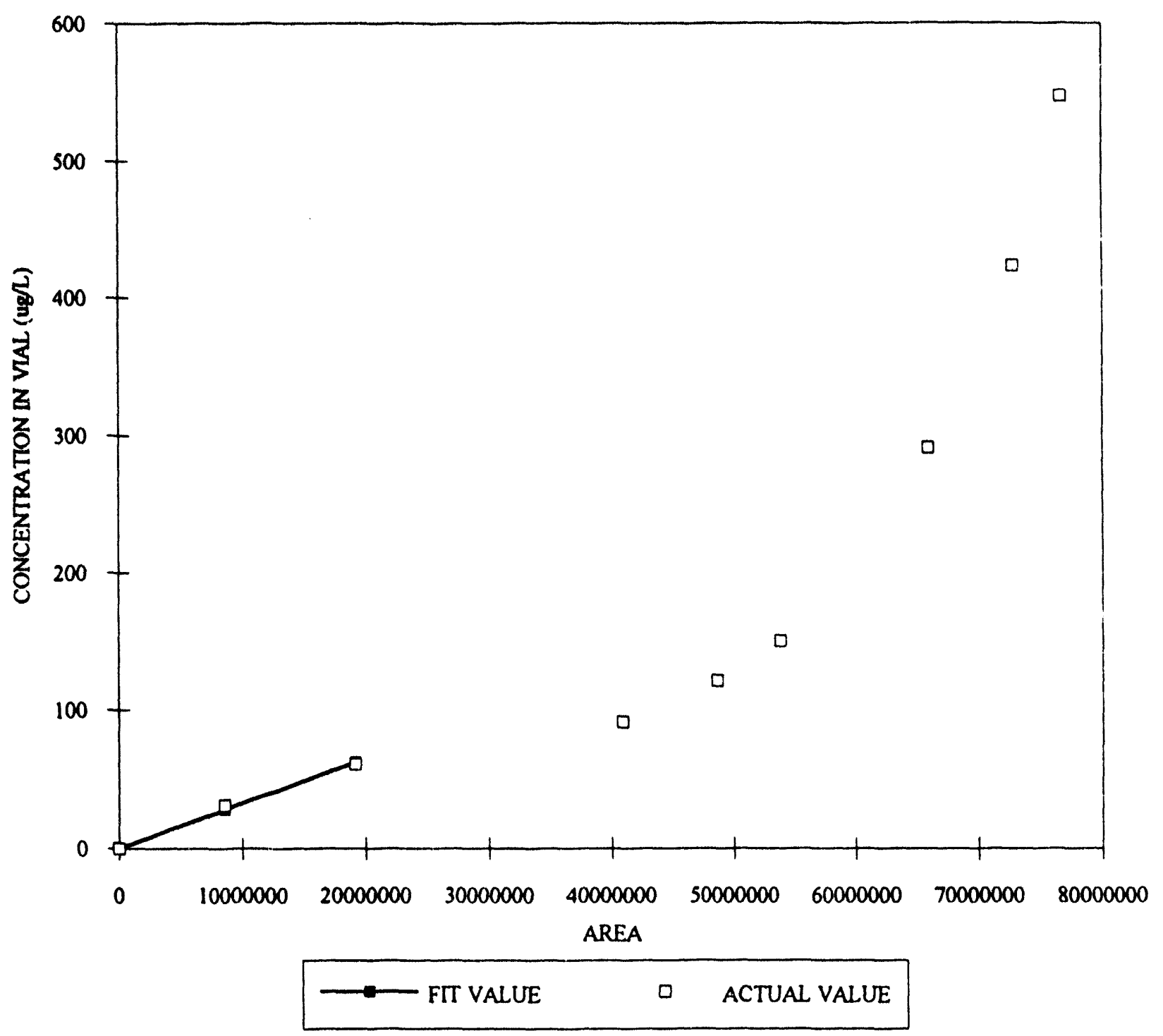




\section{Calibration Data for Depth Discrete Samples Collected from MBCSB-7}

Sample (sediment) concentrations are related to calibsation concentrations using the equation:

Sediment concentration $[\mathrm{ug} / \mathrm{g}]=$

(Standard concentration [ug/L] x $0.0075[\mathrm{~L}]$ ) / sediment mass $[\mathrm{g}]$. 


\begin{tabular}{|c|c|c|c|c|c|c|}
\hline Samples & TCE area & PCE area & Samp. Info & & TCE $\mu g / L$ & PCE $\mu_{g} / L$ \\
\hline 0 & $3.640 \mathrm{E}+03$ & $1.732 E+03$ & 0 & & 0.0 & \\
\hline so & $5.433 E+06$ & $9.131 E+06$ & STANDARD TCE & CE MDX & 62.0 & 31.0 \\
\hline 100 & $1.164 E+07$ & $2.026 E+07$ & STANDARD TCI & CE MDX & 122.0 & 61.0 \\
\hline 150 & $2.184 E+07$ & $4.123 E+07$ & STANDARD TCI & CE MDX & 182.0 & 91.0 \\
\hline 200 & $2.987 E+07$ & $4.912 E+07$ & STANDARD TC & CE MDX & 242.0 & 121.0 \\
\hline 250 & $3.916 E+07$ & $3.390 E+07$ & STANDARD TC & CE MDX & 300.0 & 150.0 \\
\hline 500 & $5.460 E+07$ & $6.569 E+07$ & STANDARD TC & CE MDX & $\$ 81.0$ & 291.0 \\
\hline 750 & $6.210 E+07$ & $7.248 E+07$ & STANDARD TC & CE MDX & 845.0 & 423.0 \\
\hline 1000 & $6.611 E+07$ & $7.634 E+07$ & STANDARD TC & CE MDX & 1094.0 & 547.0 \\
\hline & TCE & & & PCE & & \\
\hline slope & $1.065 \mathrm{E}-05$ & & slope & $3.075 E-06$ & & \\
\hline$y$ intercept & $0.000 E+\infty 0$ & & $y$ intercept & $0.000 E+\infty 0$ & & \\
\hline r2 & 0.99720 & & r2 & 0.99449 & & \\
\hline AREA & FIT VALUES & ACTUAL VALUES & AREA & FIT VALUES & ACTUAL VALUES & \\
\hline $3.640 E+03$ & 0.0 & 0.0 & $1.732 E+03$ & 0.0 & 0.0 & \\
\hline $5.433 E+06$ & 57.9 & 62.0 & $9.131 E+06$ & 28.1 & 31.0 & \\
\hline $1.164 E+07$ & 123.9 & 122.0 & $2.026 E+07$ & 62.3 & 61.0 & \\
\hline $2.184 E+07$ & & 182.0 & $4.123 E+07$ & & 91.0 & \\
\hline $2.987 E+07$ & & 242.0 & $4.912 E+07$ & & 121.0 & \\
\hline $3.916 E+07$ & & 300.0 & $5.390 E+07$ & & 150.0 & \\
\hline $5.460 E+07$ & & 581.0 & $6.569 E+07$ & & 291.0 & \\
\hline $6.210 \mathrm{E}+07$ & & 845.0 & $7.248 E+07$ & & 423.0 & \\
\hline $6.611 E+07$ & & 1094.0 & $7.634 E+07$ & & 547.0 & \\
\hline
\end{tabular}




\section{MSB-CB7 ECD/TCE}

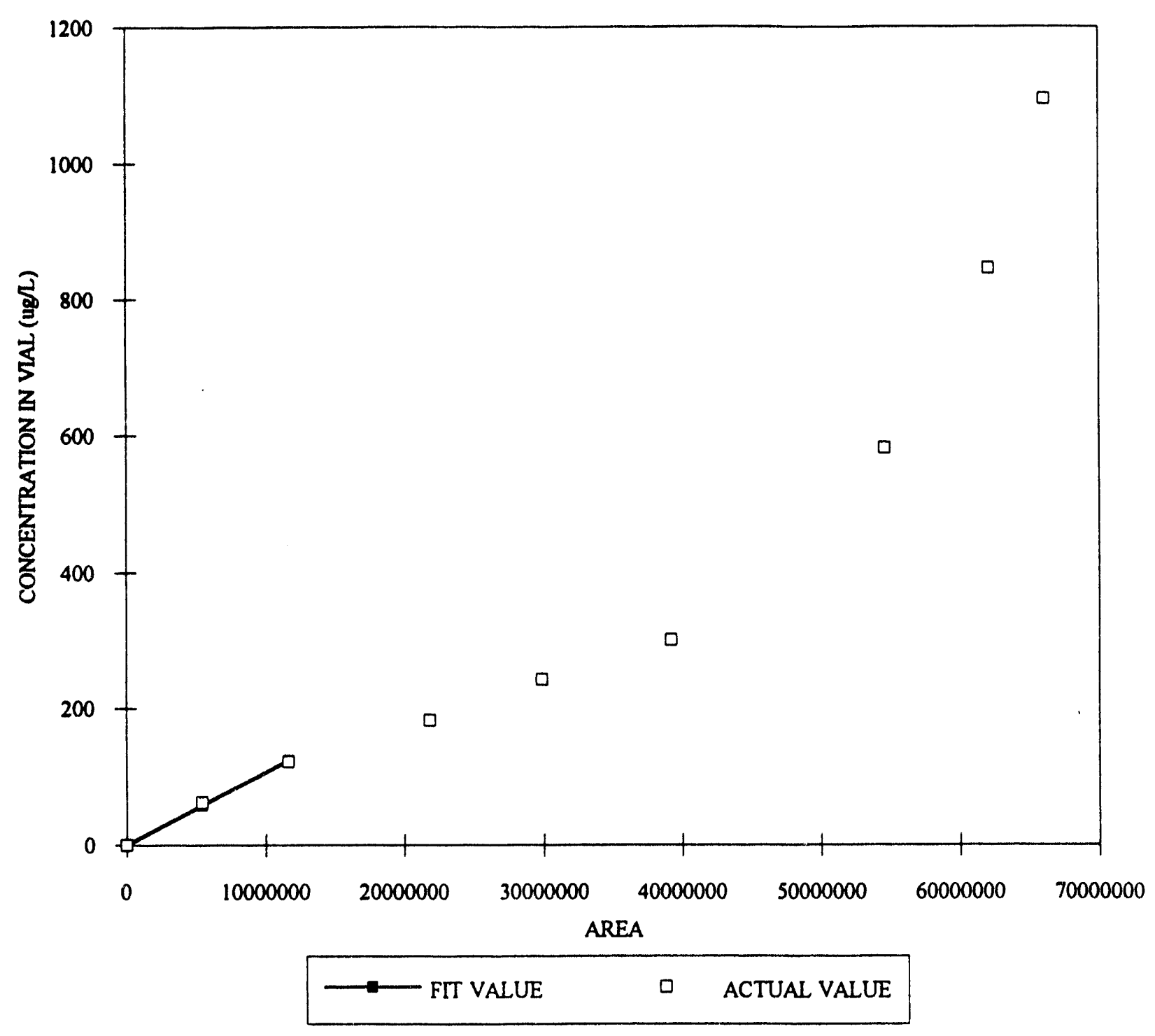


MSB-CB7 ECD/PCE

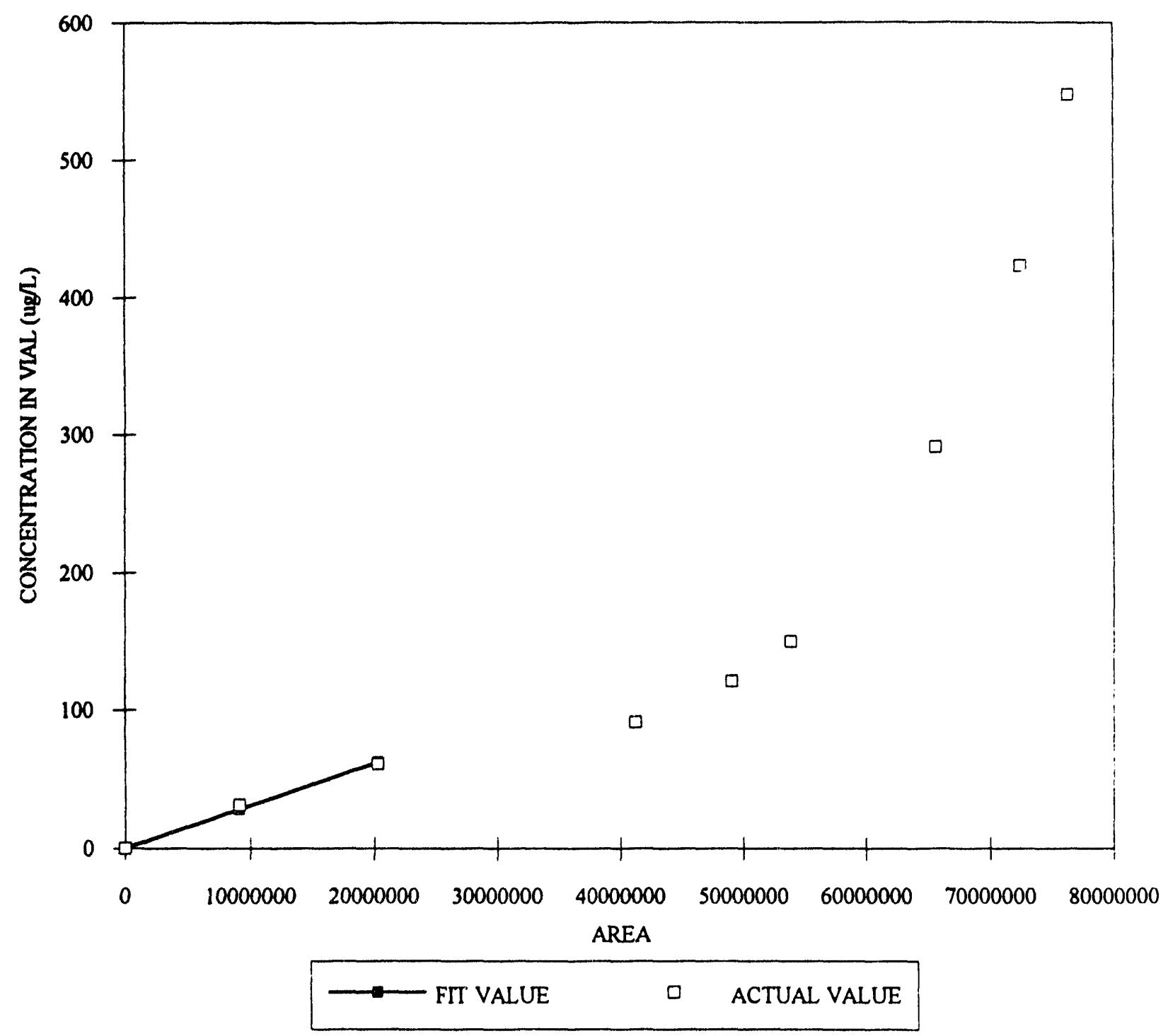



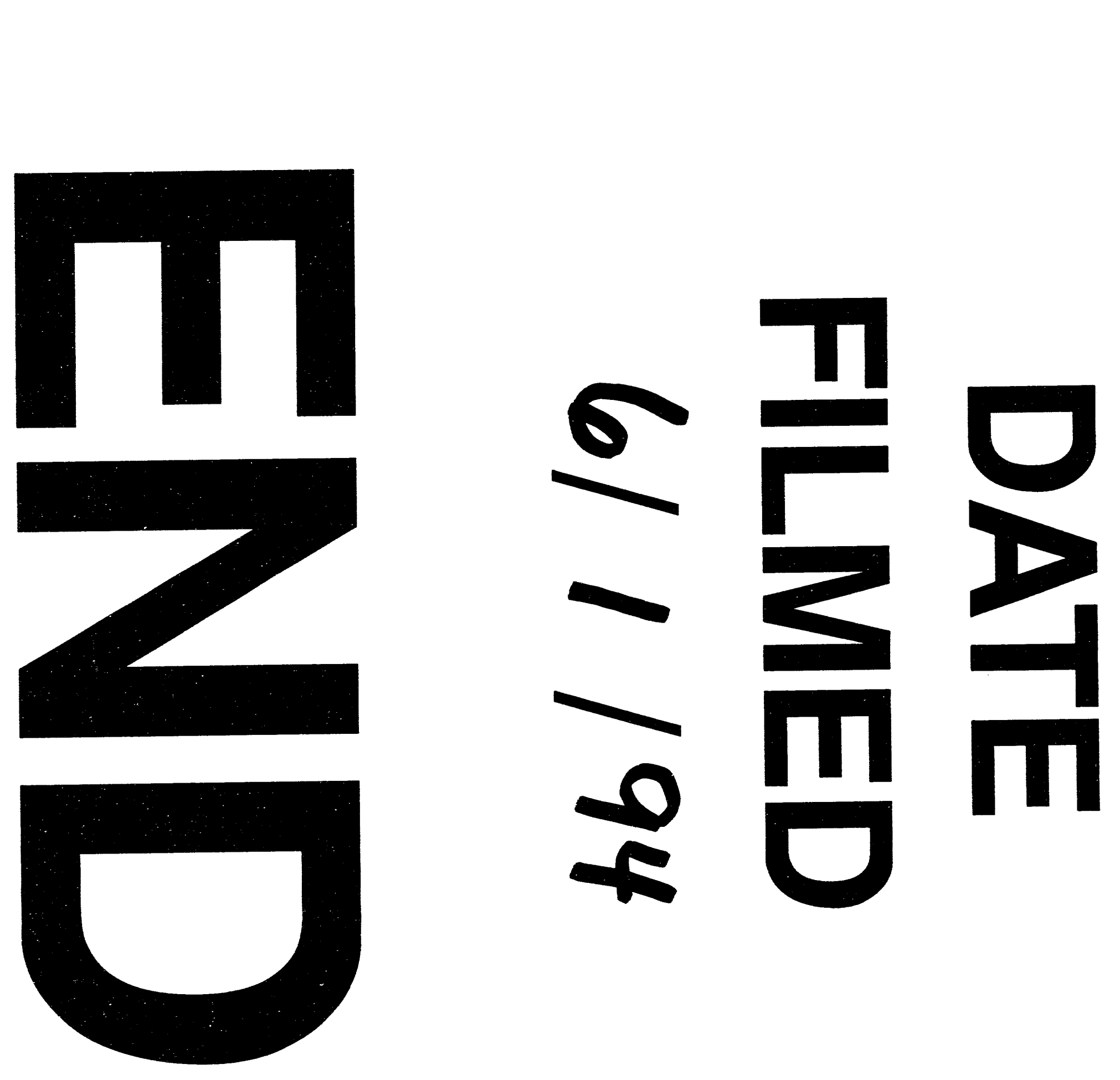


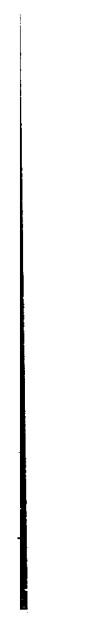

